\title{
Republic of the Philippines
}

\section{Unlocking the Potential of the Bangsamoro People through the Alternative Learning System}

October 11, 2019

GED02

EAST ASIA AND PACIFIC

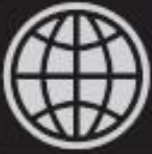


Standard Disclaimer:

This volume is a product of the staff of the International Bank for Reconstruction and Development/The World Bank. The findings, interpretations, and conclusions expressed in this paper do not necessarily reflect the views of the Executive Directors of The World Bank or the governments they represent. The World Bank does not guarantee the accuracy of the data included in this work. The boundaries, colors, denominations, and other information shown on any map in this work do not imply any judgment on the part of The World Bank concerning the legal status of any territory or the endorsement or acceptance of such boundaries.

Copyright Statement:

The material in this publication is copyrighted. Copying and/or transmitting portions or all of this work without permission may be a violation of applicable law. The International Bank for Reconstruction and Development/The World Bank encourages dissemination of its work and will normally grant permission to reproduce portions of the work promptly.

For permission to photocopy or reprint any part of this work, please send a request with complete information to the Copyright Clearance Center, Inc., 222 Rosewood Drive, Danvers, MA 01923, USA, telephone 978-750-8400, fax 978-750-4470, http://www.copyright.com/.

All other queries on rights and licenses, including subsidiary rights, should be addressed to the Office of the Publisher, The World Bank, $1818 \mathrm{H}$ Street NW, Washington, DC 20433, USA, fax 202-522-2422, e-mail pubrights@worldbank.org. 


\section{Abbreviations and Acronyms}

\begin{tabular}{|c|c|c|c|}
\hline A\&E & Accreditation and Equivalency & LAC & Learning Action Cell \\
\hline ADM & Alternative Delivery Mode & $\mathbf{L F}$ & Learning Facilitator \\
\hline $\mathbf{A L M}$ & Alternative Learning Modalities & LFS & Labor Force Survey \\
\hline ALS & Alternative Learning System & LIS & Learner Information System \\
\hline APIS & Annual Poverty Indicators Survey & $\mathbf{L V}$ & Literacy Volunteer \\
\hline ARMM & $\begin{array}{l}\text { Autonomous Region in Muslim } \\
\text { Mindanao (former BARMM) }\end{array}$ & MILF & Moro Islamic Liberation Front \\
\hline BARMM & $\begin{array}{l}\text { Bangsamoro Autonomous Region } \\
\text { in Muslim Mindanao }\end{array}$ & Misosa & $\begin{array}{l}\text { Modified In-School/Off- } \\
\text { School Approach }\end{array}$ \\
\hline BEAM & $\begin{array}{l}\text { Basic Education Assistance for } \\
\text { Mindanao }\end{array}$ & MT & Mobile Teachers \\
\hline BLP & Basic Literacy Program & MTF & Mindanao Trust Fund \\
\hline BOL & Bangsamoro Organic Law & MYDev & $\begin{array}{l}\text { Mindanao Youth for } \\
\text { Development }\end{array}$ \\
\hline CAR & Cordillera Administrative Region & NCR & National Capital Region \\
\hline CBCS & $\begin{array}{l}\text { Consortium of Bangsamoro Civil } \\
\text { Society }\end{array}$ & NER & Net Enrollment Rate \\
\hline CCT & Conditional Cash Transfer & OECD & $\begin{array}{l}\text { Organisation for Economic } \\
\text { Co-operation and } \\
\text { Development }\end{array}$ \\
\hline CLC & Community Learning Center & OHSP & Open High School Program \\
\hline DALSC & District ALS Coordinators & OSYA & Out-of-school youth and adults \\
\hline DepEd & Department of Education & PATHWAYS & $\begin{array}{l}\text { Education Pathways for Peace } \\
\text { in Mindanao }\end{array}$ \\
\hline DFAT & $\begin{array}{l}\text { Department of Foreign Affairs and } \\
\text { Trade, Australia }\end{array}$ & PESO & $\begin{array}{l}\text { Public Employment Service } \\
\text { Office }\end{array}$ \\
\hline DOLE & $\begin{array}{l}\text { Department of Labor and } \\
\text { Employment }\end{array}$ & PHP & Philippines Peso \\
\hline DSWD & $\begin{array}{l}\text { Department of Social Welfare } \\
\text { Development }\end{array}$ & PR & Participation rates \\
\hline EBEIS & $\begin{array}{l}\text { Basic Education Information } \\
\text { System }\end{array}$ & PSA & Philippine Statistics Authority \\
\hline EST & Education Skills Training & RPL & Recognition of Prior Learning \\
\hline FLEMMS & $\begin{array}{l}\text { Functional Literacy, Education, } \\
\text { Mass Media Survey }\end{array}$ & SEAMEO & $\begin{array}{l}\text { Southeast Asian Ministers of } \\
\text { Education Organization }\end{array}$ \\
\hline FLT & functional literacy test & SES & Socioemotional Skills \\
\hline GED & General Education Development & STEP & $\begin{array}{l}\text { Skills Toward Employment } \\
\text { and Productivity }\end{array}$ \\
\hline ILA & individual learning agreement & TESDA & $\begin{array}{l}\text { Technical Education Skills } \\
\text { Development Authority }\end{array}$ \\
\hline IM & Instructional Manager & TVET & $\begin{array}{l}\text { Technical and Vocational } \\
\text { Education and Training }\end{array}$ \\
\hline ACT & $\begin{array}{l}\text { Instructional Management by } \\
\text { Parents, Community and Teachers }\end{array}$ & AID & $\begin{array}{l}\text { United States Agency for } \\
\text { International Development }\end{array}$ \\
\hline HS & Junior High School & WB & World Bank \\
\hline
\end{tabular}




\section{Table of Contents}

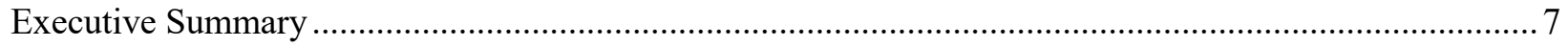

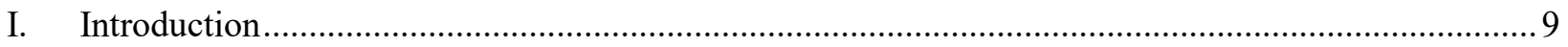

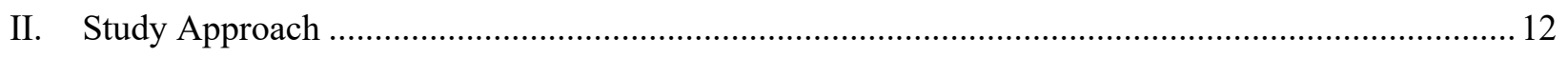

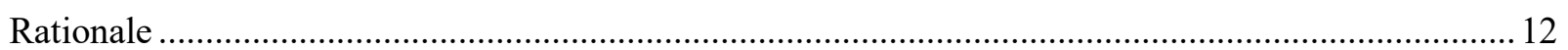

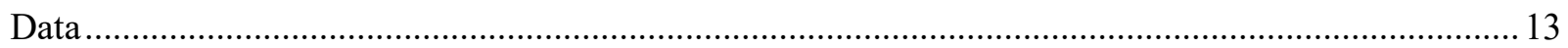

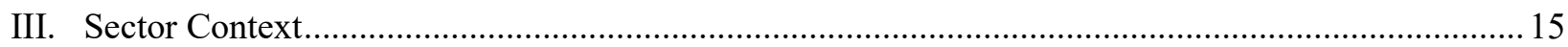

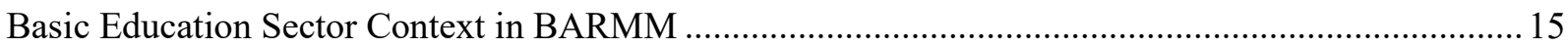

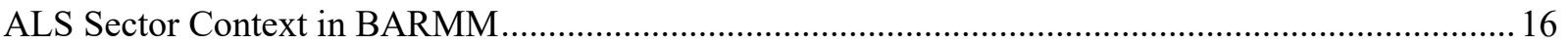

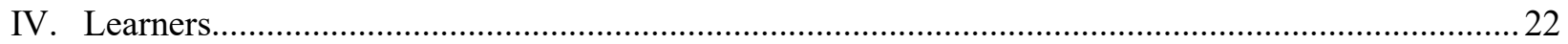

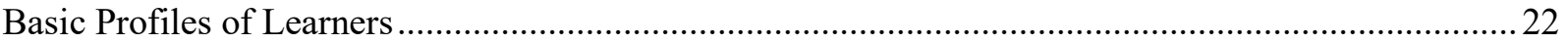

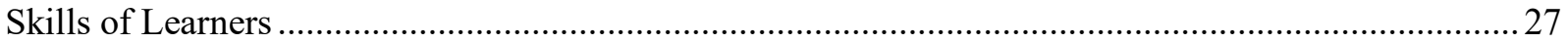

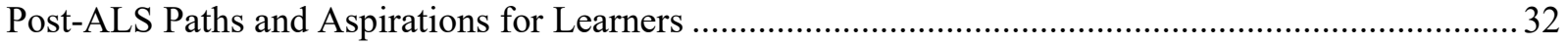

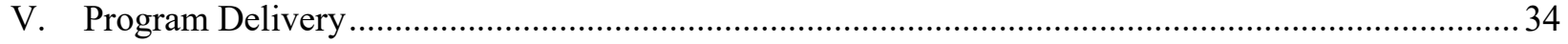

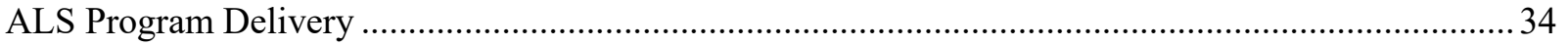

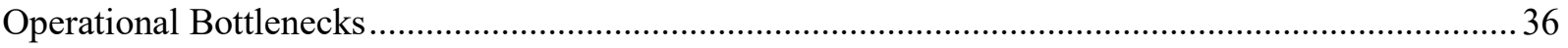

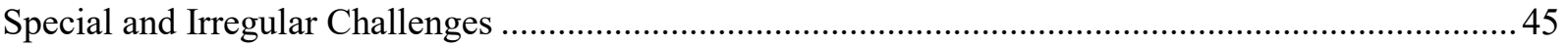

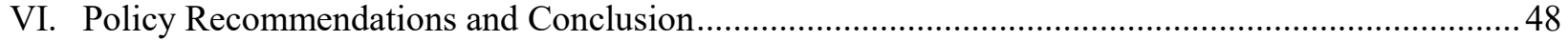

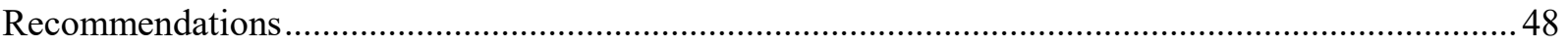

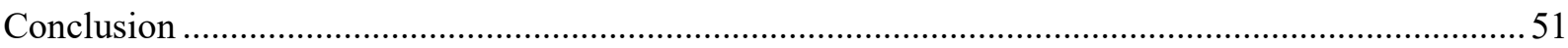

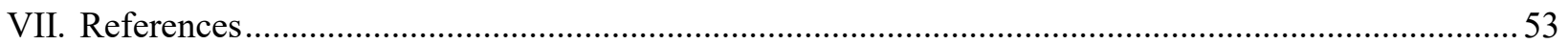




\section{List of Figures, Tables, and Boxes}

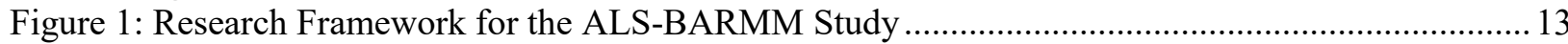

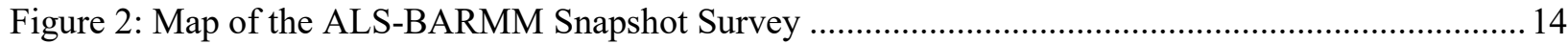

Figure 3: Net Enrollment Rate Trend of BARMM and Nationwide, 2009-2017 ................................ 16

Figure 4: School Completion Rate Trend of BARMM and Nationwide, 2009-2017 .............................. 16

Figure 5: Comparison of the Number of OSYA (below age 30) Using LFS and Listahanan..................... 18

Figure 6: Trends in ALS Performance, Nationwide ..........................................................................20

Figure 7: Share of Passers Among Those Who Took A\&E Exams for Elementary and Secondary Levels

Figure 8: Share of Learners by ALS Program, BARMM (Inner ring), and Nationwide (Outer ring) ........22

Figure 9: Age of Learners by ALS Program and Gender ................................................................22

Figure 10: Distribution of ALS Learners by Age and Marital Status ...................................................23

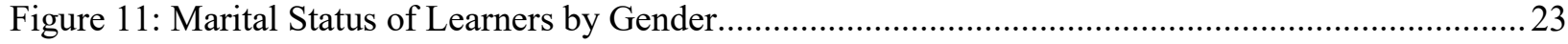

Figure 12: Highest Educational Level Completed Before ALS for Younger and Older ALS Learners (\%)

.

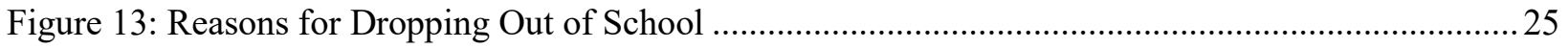

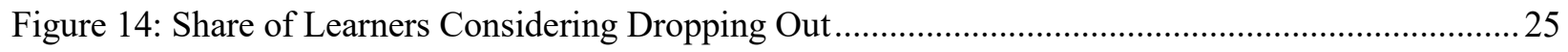

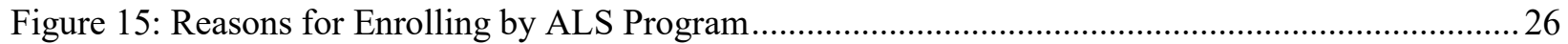

Figure 16: Share of ALS Learners Who Attended, Completed, Took the A\&E Test and Passed ..............26

Figure 17: Correct Responses (\%) by BARMM ALS Learners in Comparison with Total OSYA, Ages 16-

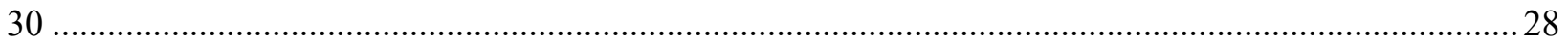

Figure 18: Correct Responses (\%) by BARMM ALS Learners by ALS Programs, Ages 16-30 ..............28

Figure 19: Average BARMM Scores in Socioemotional Skills Compared to Total OSYA and Population,

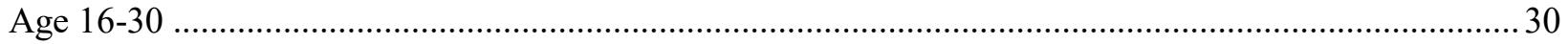

Figure 20: Average BARMM Scores in Socioemotional Skills by ALS Programs, Age 16-30................30

Figure 21: Distribution of Socioemotional Skills of ALS Learners in BARMM by Various Groups........ 30

Figure 22: Share of Facilitators Providing Skills Training for Learners................................................. 32

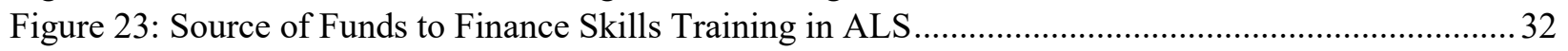

Figure 24: Immediate Next Steps After ALS Reported by ALS Learners in BARMM .......................... 32

Figure 25: Long-term Career Goals of ALS Learners in BARMM ..................................................... 33

Figure 26: Share of Past ALS Learners Pursuing Further Education/Training After ALS ........................ 34

Figure 27: Share of Past ALS Learners' Employment Status After ALS ................................................ 34

Figure 28: ALS and its System Components for Effective Program Delivery ......................................... 35

Figure 29: Suggestions for Improvement as Reported by Learners in BARMM by ALS Program ........... 35

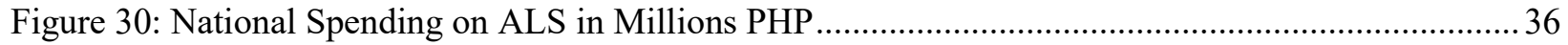

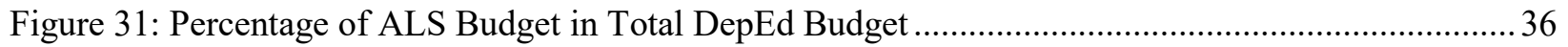

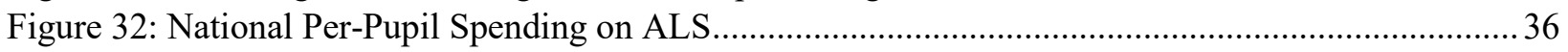

Figure 33: Number of ALS Field Implementers by Regions, 2018 ................................................. 37

Figure 34: Number of ALS Facilitators as Shares to OSYA and ALS Enrollers by Regio, 2018 ............. 37

Figure 35: Levels of Education Among ALS Facilitators in BARMM .................................................. 38

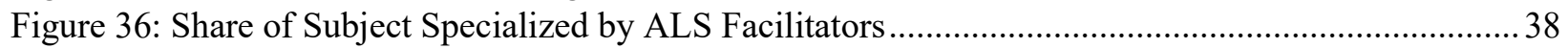

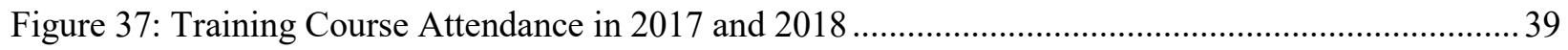

Figure 38: Sources of Funding for In-service Training Courses........................................................... 39

Figure 39: Reported Availability of ALS Learning Modules by Subprogram, 2018 (\% of facilitators) .... 40

Figure 40: Facilitator Expenses by Type in BARMM in 2018 (\% of Facilitators Who Reported Spending a

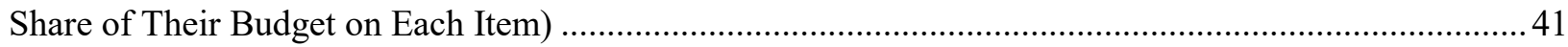

Figure 41: Amount Paid Per Transaction on Average in BARMM, 2018 (PHP) .................................... 42 
Figure 42: Total Weekly Spending by ALS Facilitators in BARMM, 2018 (PHP) ............................... 43

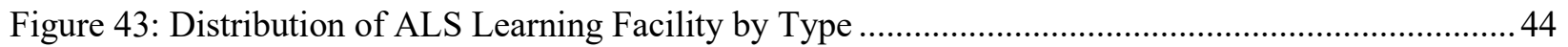

Figure 44: Number of Monitoring Visits to ALS Activities by Agent per Year ......................................45

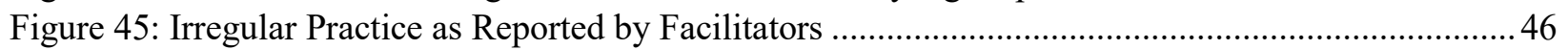

Figure 46: Irregular Practice as Reported by Facilitators II ................................................................. 46

Table 1: Summary of the ALS-BARMM Snapshot Survey Samples .................................................... 14

Table 2: Summary of Out-of-School Population: Nationwide and in BARMM ..................................... 17

Table 3: Estimated OSYA, ALS Participants, and ALS Participation Rate ............................................20

Table 4: ALS Enrollment, Completion, and Completion Rate of BARMM and National Average...........21

Table 5: Profiles of ALS Learner Sample in BARMM ...................................................................2

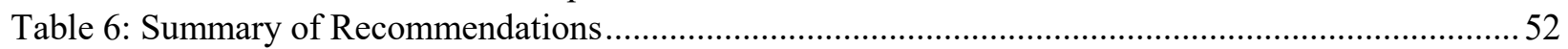

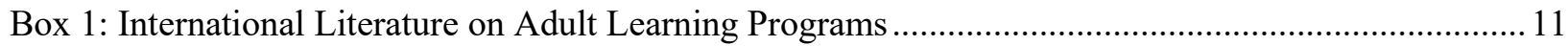

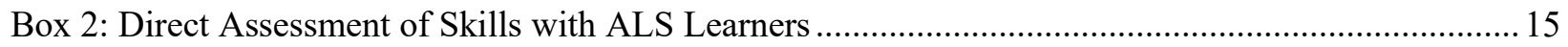

Box 3: Practical Approaches to Quantify Prospective Beneficiaries for ALS........................................ 17

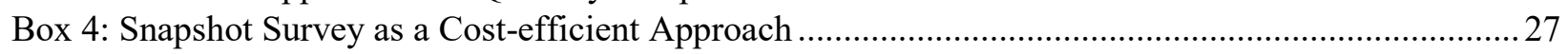

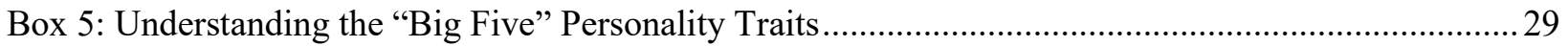

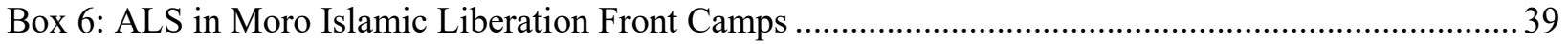

Box 7: "Ghosts" in ALS: Circumventing ALS service Delivery Standards in BARMM..........................47

\section{Acknowledgement:}

This study and survey were led by Takiko Igarashi (Education Specialist), Nicholas Tenazas (Consultant), and Pablo Acosta (Senior Economist).

The team is grateful to Mr. Mohagher Iqbal, Minister of Basic, Higher and Technical Education (MBHTE); Ms. G.H. Ambat, Assistant Secretary for Department of Education (DepEd); Atty. Rasol Mitmug, Former Regional Secretary of DepEd ARMM; Mr. Majuni Maddi, Assistant Secretary for MBHTE; the ALS Taskforce of DepEd; as well as their colleagues at DepEd and MBHTE for their wholehearted support and guidance. We are also extremely grateful to the Consortium for Bangsamoro Civil Society and the Maguindanao II School Division Office for their insights and support in the fieldwork and consultation. We also appreciate useful and technical input from Mr. Philip Purnell (SEAMEO INNOTECH), Ms. Victoria Levin (WB), Ms. Melissa Adelman (WB), as well as the Working Group for the Bangsamoro ALS Roadmap including SEAMEO-INNOTECH, UNICEF, BRAC, and BARMM MBHTE officials. The team is highly grateful for the overall guidance from Ms. Mara Warwick (Country Director, WB), Mr. Tobias Linden (Education Global Practice Manger, WB), and Mr. Philip O'Keefe (Social Protection and Jobs Global Practice Manger, WB). The team gives special thanks to Ms. Jessica Arends for outstanding editorial assistance, as well as Ms. Anna Alejo, Ms. Azel Gorne, Ms. Yolanda Azarcon, Ms. Regina Calzado, and Mr. Ed Alvinez for their outstanding support.

This study received the support of the Australian Government through the Australia-World Bank Philippines Development Trust Fund. The opinions and conclusions expressed in this paper are the authors' and do not necessarily reflect positions of the World Bank and its member governments. Any remaining errors are of the authors. 


\section{Executive Summary}

In 2018, after decades of conflicts, peace negotiation finally concluded with the creation of the Bangsamoro Autonomous Region in Muslim Mindanao (BARMM), recently approved through the 2019 Bangsamoro autonomy plebiscite, which is envisioned to have greater autonomy, more resources, and larger territory than its predecessor. The Bangsamoro People have high expectation to seize dividends of peace from this political transition process.

BARMM has faced serious challenges in both access and quality of basic education even while the Philippines as a whole has made remarkable progress in the basic education reform over the last decade. In BARMM, only 1 out of every 10 students who began primary education completed junior high school (JHS) on time. Out-of-school youth and adults (OSYA) have thus accumulated over the generations. The share of OSYA among the 16-30 years-old population has been close to 45 percent in BARMM, double the national average. These figures point to a crisis in the delivery of basic education in the BARMM area - a crisis which needs to be addressed as a first priority to keep children in school and to provide better quality education to them. This will not only increase achievement but also abate the OSYA population.

Complementary to the efforts in providing quality education for all Filipino children, the Department of Education (DepEd) has been operating the Alternative Learning System (ALS) to offer second-chance learning for OSYA for the past few decades throughout the country. ALS consists of the basic literacy program (BLP) and the accreditation and equivalency (A\&E) programs, which are often accompanied with contextualized livelihood skills training. BLP aims to eradicate illiteracy. A\&E programs target people who are basically independent learners but did not complete the formal elementary or JHS levels. Official certificates equivalent to formal school diplomas can be earned if learners could pass the A\&E exams.

In 2018, 840,000 enrolled in ALS nationally, out of which 25,000 enrolled in BARMM. In BARMM, 40 percent enrolled in BLP, and the rest took the A\&E elementary and secondary programs, in sharp contrast to 75 percent of the learners enrolling in the A\&E secondary program across the country.

The World Bank has undertaken series of evaluations of ALS in collaboration with the DepEd across the country since 2013. The findings show significant impacts on labor market outcomes among ALS learners who could pass the A\&E exam. Also, it was found that ALS learners utilize the official A\&E certificates to pursue college, vocational training, or formal employment. However, the research also identified key impediments to effective program delivery which have led to low system effectiveness and efficiency. Moreover, the international evidence suggests that ALS-type programs generally have modest outcomes for program participants, so strenuous efforts are needed to maximum the impact of ALS.

The previous evaluations of ALS have not to date covered the BARMM area. This report fills that gap and aims to provide the new BARMM government with essential evidence for designing and undertaking reform interventions to improve the program delivery for its beneficiaries.

\section{Main Findings:}

- In 2018, only 5 percent of OSYA between ages 16 and 30 participated in ALS, compared to the national average of 13 percent. 35 percent of the ALS enrollees graduated in BARMM, which was about half of the national average. The A\&E exam pass rate for BARMM improved from 2016 to 2018 is 20 percentage points behind the national average.

- ALS learners, particularly females, struggle in gaining own family's understanding on attending both formal schools and ALS programs. Also, 2 out of every 3 dropouts were never followed up by any school staff or teachers after they dropped out in BARMM. 
- ALS learners in BARMM show lower reading and math skills, as well as socioemotional skills on average as compared with the national average score of OSYA of the same age cohort.

- ALS learners rarely receive support to make good career choices after graduating from ALS. Also, less than half of the ALS facilitators provided skills training for their own learners in 2018 in BARMM.

- Basic supplies, learning modules, operational budgets, and learning facilities for ALS suffer from serious deficiency in BARMM. This challenge is common nationwide, but the level of deficiencies is more serious and urgent in BARMM. While the Government has rightly prioritized spending in basic education (where per-pupil spending has more than doubled over the past decade in real terms), perlearner spending on ALS has declined in real terms.

- There is a severe lack of ALS teachers ('ALS learning facilitators'), both in number and specialization, and both existing and incoming ALS teachers have had limited training. In BARMM, the ratio of OSYA to a facilitator is 1,800 to 1 , by far the highest of all regions, and 10 percent of existing teachers will retire in next five years in the region.

\section{Recommendations:}

Improving the quality of basic education will help eventually reduce the large number of OSYA. Significant changes need to be made to improve the quality and effectiveness of the ALS system as it operates in BARMM. Making these changes should be given the highest priority, after which efforts to expand the program can be undertaken. The report suggests the following recommendations.

A. Level up the overall program delivery in BARMM. The BARMM Strategic Roadmap for Alternative Learning Modalities (or ALM, of which ALS is a core component), which provides priority actions and activities with specific timelines, could be the important guide to improve the ALS program delivery in BARMM.

B. Boost capacity building programs for ALS teachers in BARMM. Both existing and incoming ALS need better quality training, particularly training focused on pedagogical approaches in ALS, as well as the new K-12 Basic Education ALS curriculum in the short term. In medium term, localized peerlearning programs could be introduced for ALS teachers to promote their continuous professional development.

C. Improve approaches to reach out OSYA in BARMM as a medium-term objective. Targeted approaches through using more accurate data could be introduced for community mapping and advocacy to reach out OSYA efficiently.

D. Respond to special challenges in BARMM. On-site supervision needs to be intensified immediately to end irregular practices. In conducting regular program audits, engagement with the third parties, such as civil society groups and local communities, as well as use of technology could considered.

E. Improve the delivery of quality ALS programs across the Philippines. National public spending on ALS is critical to address persistent supply-side bottlenecks and enable the system-wide reform that will flow from the ALS Version 2.0. Efforts by DepEd to provide a policy environment to harmonize the formal school education, ALS, and ADM remain important.

F. Lay out foundation to strengthen ALS for future. Looking forward, DepEd could strengthen Recognition of Prior Learning (RPL), for assessing enrollers' prior learning/skills and customizing learning plans; and Career Consultation or information for ALS learners for making informed decisions about their future. In the medium- to long-term, development of the Modular Learning and MicroCertification Systems through digitalization needs to be explored through learning from international good practices. 


\section{Introduction}

Decades of peace negotiations between the rebel groups in Mindanao and the Philippine government finally reached an important milestone with the Bangsamoro Organic Law (BOL) signed by President Duterte in July 2018. Subsequently, the Bangsamoro autonomy plebiscite held early 2019 saw most residents in the region vote in favor of the BOL creating the Bangsamoro Autonomous Region in Muslim Mindanao (BARMM). ${ }^{1}$ The new region is envisioned to have broader autonomy, more power and resources, and larger territory than its predecessor. Bangsamoro people have high expectation for the coming political transition process to seize dividends of peace.

However, several decades of armed conflicts and civil unrest have greatly affected social services, placing the region at the lowest point on any social sector indicators in the Philippines. Today, while almost all Filipino children to start primary education at age six, only 70 percent start formal schooling in BARMM on time. Similarly, 80 percent enroll in junior high schools (JHS) on time nationwide, but only 30 percent of children who live in BARMM enter JHS on-time. Close to half of the children who enrolled in elementary schools drop out before graduation, and the same pattern continues at the JHS level for various reasons. Consequently, only 1 out of every 10 students who began primary education in BARMM graduate from JHS on time. Despite the remarkable progress in the country's basic education system reforms over the last decade, BARMM has been facing persistent and serious challenges.

This magnitude of school dropouts in BARMM has resulted in a high number of youth and adults who have limited education, literacy, and skills. The percentage of out-of-school youths and adults (OSYA) between ages 16-24 across the country was about 20 percent, but the OSYA share in BARMM was over 40 percent in 2018, which is by far the highest among all regions. Among young adults age 25-30 years, the share is 60 percent surpassing those who have successfully completed JHS. In short, the majority of the young and productive population in BARMM may critically lack necessary skills and thus face limited prospects in the labor market. These figures point to a crisis in the delivery of basic education in the BARMM area - a crisis which needs to be addressed as a first priority to keep children in school and to provide better quality education to them. This will not only increase achievement but also abate the OSYA population.

Complementary to the efforts in providing quality education for all Filipino children, the Department of Education provides the Alternative Learning System (ALS), which is an integral component of the Philippine's basic education system for youths and adults who did not complete basic education. ALS includes programs such as Basic Literacy Program (BLP) and Accreditation \& Equivalency for Elementary and Secondary Level (A\&E EL and SL) Program, which are often accompanied by locally-contextualized livelihood skills training programs. In principle, ALS programs are open to anyone who meets the eligibility condition, which is the age restriction at entry. These programs are carried out in a span of ten months in a year ${ }^{2}$. Learners are assessed on their prior-learning levels and supported by ALS facilitators to develop own individual learning agreements (ILA). If learners can achieve their respective ILAs through attending learning sessions and self-learning between the sessions, then they are regarded as completers of the program. But in order to earn the official certificates that are equivalent to the formal schools, they are required to pass the national A\&E exam, which is offered once a year. With the official A\&E certificates, ALS learners are able to pursue further education including junior and senior high schools, post-secondary technical and vocational education, or formal sector employment, which requires junior secondary education completion. 
ALS programs has been implemented by DepEd and other partners for a few decades in all regions including BARMM, providing an important second chance for OSYA to return to the human capital development path. In 2018, over 840,000 enrolled in ALS nationally, out of which 25,000 enrolled in BARMM. Given the scale of ALS, it is an important complement to the formal education system, and a key mechanism for helping young people acquire the education qualifications for the labor market, which they could not acquire through the formal system.

With full implementation of the K-12 basic education system in 2016 ${ }^{3}$, DepEd has put forward key reforms to upgrade of ALS programs. In 2019, DepEd has rolled out the new ALS K-12 Basic Education Curriculum nationally including BARMM, which is also known as "ALS Version 2.0". With the alignment of the ALS curriculum, reforms and realignment of ALS' system components have been undertaken. This includes retraining of ALS facilitators, learning modules, assessments, certification system, delivery modes, and monitoring and evaluation mechanism. In addition, DepEd has piloted the ALS Education Skills Training (ALS-EST), which provides ALS learners with both academic courses and practical skills training for immediate employment in collaboration with technical vocational schools and other partners since 2018.

Across developing countries, various non-formal education programs have been offered for adults and youth who never had education or left without achieving a qualification in order to meet their education needs. These programs vary very widely from teaching basic literacy, providing equivalency education systems, assessing and certifying learning and skills, and providing integrated academic learning and skills training. For example, Indonesia provides a three-tier non-formal equivalency education system ${ }^{1}$ aligned to the country's formal school education levels, which is quite similar to the A\&E programs of ALS in the Philippines. Also, Mexico's Education Model for Life and Work (Modelo Educación para la Vida y el Trabajo, or MEVyT) operates basic literacy learning programs accompanied with skills training, which is also similar to the ALS-EST program mentioned above. MEVyt also offers tailor-made modules for Mexico's many indigenous ethnic groups to make education accessible in local indigenous languages and acceptable to local ethnic communities (Hanemann, 2017). Furthermore, non-formal education has been increasingly more relevant in conflict-affected areas to address the immediate needs of the displaced peoples or refugees who left schools without completion or lost their qualification documents. By assessing and certifying prior learning and skills acquired already through these non-formal education programs, they are able to re-enter formal education and training, or integrate into the society and world of work in the host countries (Singh, Idris, and Maysoun, 2018).

Globally, adult learning programs targeting out-of-school youth and adults face a common set of challenges. These challenges include a higher opportunity cost for adult learners, as well as reduced brain plasticity compared to school children, which inhibits the acquisition of new knowledge and skills, and uncertainty perceived among prospective participants regarding the economic returns to completing learning programs (Aker and Sawyer, 2017). Also, research suggests that deficiencies of socio-emotional skills among out-of-school youngsters (e.g. adaptability, sociability, effort, attention, persistence selfefficacy, self-regulation, outlook on life, etc.) may impede outcomes of learning interventions while their academic aptitudes may be developed as comparable to school completers (Heckman, et. al, 2011; Heckman, et. al, 2012; Rajasekaran and Reyes, 2019). (See Box 1)

Despite an increasing focus on adult learning and emerging evidence on individual and social benefits of adult education programs, evidence on "what works' is still generally limited. Most recent

\footnotetext{
${ }^{1}$ Indonesia's equivalence education program consists of Paket A, Paket B and Paket C. Paket A is equivalent to elementary school; Paket B to junior high school; and Paket C to senior high school education. (Herlyna, Mukhtar, \& Sari, 2019)
} 
neuroscientific evidence shows that adults' brains are actually malleable and have the plasticity to learn efficiently prior to their 70s (Thomas et. al, 2019), but adults need to be taught in ways that they can effectively learn in proper sequencing and quality instruction (Aker and Sawyer, 2017). Research finds positive grains for learners in increased literacy and household welfare from adult-learning interventions in South Asia and Africa (Blunch and Pörtner, 2011; Aker and Ksoll, 2015; Banerji, et. al, 2017; Sawada, et. al, 2017, etc). Furthermore, the 2019 OECD report describes seven action points to create more and better learning opportunities for low-educated and skilled adults given the changing labor market, through reviewing various adult learning programs and policies, particularly those in advanced economy.

DepEd and the World Bank have partnered to deliver a series of program evaluations on ALS since 2013, which has led to ALS policy enhancement and reforms. This collaboration includes a series of surveys across the country: the 2013 ALS Survey in the Greater Metro Manila Area, the 2015 ALS National Data Collection, and the 2017 ALS Snapshot Survey. Findings from these studies suggest that there are significant benefits, particularly higher earnings and better labor market opportunities, among ALS learners, particularly if they are able to pass the A\&E. However, the program has been met with several key challenges both at the policy and field levels (Yamauchi, Igarashi, Tenazas, \& Tiongco, 2016; Igarashi, 2018).

The previous evaluations of ALS have not to date covered the BARMM area, so this study fills that gap and aims to provide the new BARMM government essential evidence based on new data for designing and undertaking reform interventions to improve the program delivery for its beneficiaries. After this introduction, the second section provides details on the methodology and data. The third section discusses the situation of BARMM's formal school education sector and ALS operation. The fourth and fifth sections focus on challenges on the learners (demand-side) and the program delivery (supply-side), respectively. The final section provides conclusions and recommendations.

Box 1: International Literature on Adult Learning Programs

A common set of challenges have been highlighted from adult learning programs conducted in both advanced and developing economies. The first challenge is biological or related to neuroscience. Adults' learning process differs from those of children and young adolescents. Adult brains are less malleable and have begun deteriorating, making it more difficult for adults to assimilate new information and learn new skills. However, the most recent research shows that, if proper stimulation is provided, adults can develop skills like literacy and numeracy but at a slower pace compared to children. Thus, more practice is required for adults to achieve automaticity and eventually comprehension to develop literacy.

The second challenge is socioeconomic, as adults who participate in education programs bear a higher opportunity cost in terms of foregone revenue or deferred domestic responsibilities. For adults, time spent in a classroom is time not spent earning income or managing a household. Also, high uncertainty about the economic returns to education affect adults' decision making and capacity for learning.

The third challenge is related to non-cognitive or socioemotional skills. The international research analyzing the General Education Program (GED), an equivalency testing program for high school dropouts in the United States, found that there were minimal benefits among the majority of the GED test graduates, in terms of gaining economic opportunities or using the certificate as a path to pursue higher academic credentials. The research explains that while the GED test graduates are able to demonstrate scholastic aptitudes in reading, writing, language, and mathematics as equivalent to high school graduates, their opportunities are still limited because they have lower socioemotional skills (or non-cognitive skills) such as persistence, motivation, and reliability.

More recent work suggests a set of possible approaches to overcome these challenges based on the empirical evidence. This include (i) proper sequencing of subjects and learning contents; (ii) greater emphasis on practice and repetition; (iii) a focus on developing high-order thinking (or meta-cognitive) skills; (iv) offering 
incentives and support to enable adult learners to manage high opportunity costs; (v) proper learning environment to stimulate motivation with a mechanism to provide timely feedback on performance; (vi) ensuring that teachers understand the ways in which techniques for teaching adults (andragogy) differ from techniques for teaching children (pedagogy) and (vii) integration of socio-emotional learning through mentoring and psychological support.

In addition, the Organization for Economic Cooperation and Development (OECD) has published a report on how to engage low-skilled adults in learning at the nexus of the Fourth Industrial Revolution (or Industry 4.0) based of reviews of adult learning programs and related policies across the world. Seven action points are highlighted to create better learning opportunities as follows: ${ }^{4}$

- Action 1: Find creative ways to reach potential learners: reach places where target adults spend time frequently.

- Action 2: Offer holistic and personalized advice and guidance: provide guidance services by qualified staff who are able to tailor available services and provide holistic advice.

- Action 3: Create interesting and relevant learning opportunities: design programs based on how adults learn - be practical, problem-oriented, and closely linked to the context of the learner - when designing learning programs.

- Action 4: Recognize existing skills: provide access to skill recognition procedures and to navigate and prepare for the recognition process.

- Action 5: Provide modular learning opportunities: choose modules from different types of provisions to create individual learning paths and provide adults with micro-credentials allowing them to combine these to achieve a full qualification.

- Action 6: Give people time off to participate in learning: give individuals the right to education and training leave, either by law, through collective agreements or bilateral agreements with employers.

- Action 7: Provide financial support for all training costs: provide targeted financial incentives for low-skilled adults which steer their training choices toward skills in demand.

(Sources: Thomas et. al, 2019; Aker and Sawyer, 2017; Heckman, et. al, 2011; Heckman, et. al, 2012;

Rajasekaran and Reyes, 2019; Banerji, et. al, 2017; OECD, 2019, etc)

\section{Study Approach}

\section{Rationale}

The objective of this study is to provide independent and objective data for enhancing policy and programs to improve the ALS programs in BARMM. There have not been any systematic evaluations nor sufficient data on ALS in BARMM while the World Bank in partnership with DepEd has conducted a series of surveys and studies to assess the implementation of ALS programs nationwide. This study focuses on developing wholistic understanding on the following three areas: (a) the context of the formal basic education sector and ALS, (b) the ALS learners, such as their characteristics and skills profiles, as well as their paths after graduating from ALS; and (c) the program delivery, particularly impediments to effective operations such as teachers, learning modules, operational expenses, learning environment, and program monitoring), as well as region-specific challenges (Figure 1). This study was consulted with the former DepEd ARMM Regional Office (current Ministry of Basic, Higher and Technical Education for BARMM), DepEd Central Office, and other local and international experts. As much as possible, the findings from the past studies the WB has conducted across the country are used as benchmarks to discuss the findings. 
Figure 1: Research Framework for the ALS-BARMM Study

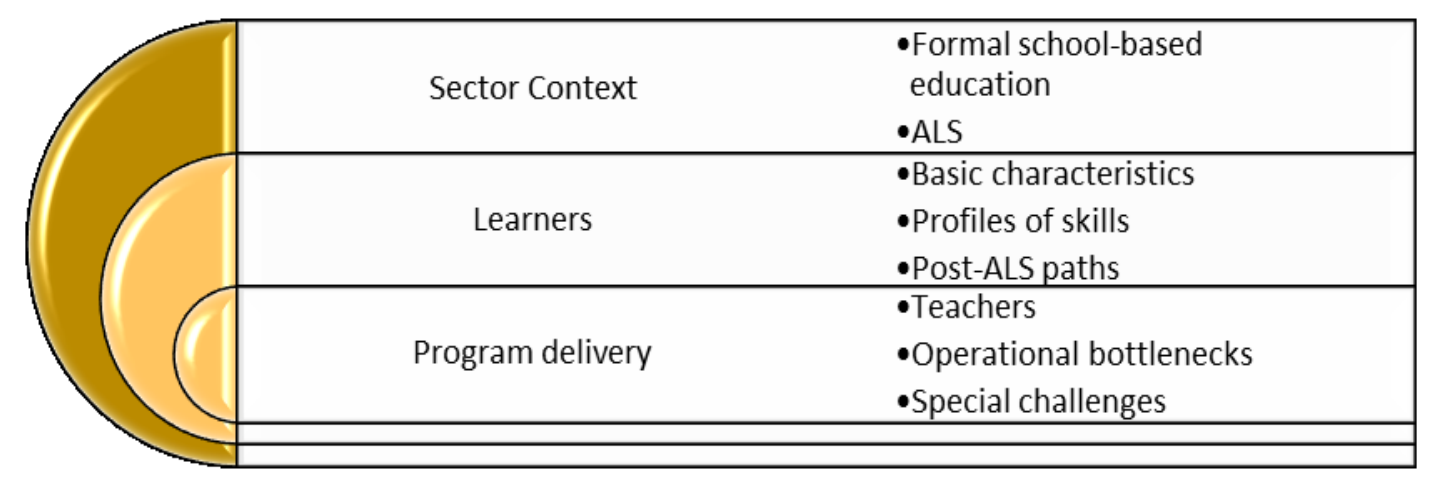

Source: Authors' analysis.

\section{Data}

\section{Official data}

Data for BARMM has been generally scarce, and when it comes to ALS, the availability of the data has been the most challenging in terms of both availability and reliability. Even general data extracted from the Basic Education Information System (EBEIS) sometimes contains faulty figures, which can be attributable to the mode of data collection and absence of a validation mechanism. This challenge may be more serious for the data that was collected in BARMM as evidence from the 2015 World Bank Report describes "ghost" schools, teachers, and students in its education system.

Given the challenge, we drew from mixed data sources. First, the DepEd's official data extracted from the EBEIS and the Learner Information System (LIS) is used as it provides the numbers of ALS enrollers, completers, and passers of Accreditation \& Equivalency test (A\&E test), as well as ALS learning facilitators. The administrative data was obtained from DepEd Central and the former ARMM region to triangulate the values. Second, the national household survey data was used mainly describe the population by age group, educational attainment level, and literacy skills. Specifically, the raw survey data from the Philippines Statistics Authority (PSA), the Department of Social and Welfare Development (DSWD), and the World Bank was utilized. Third, a primary data collection, the 2018 ALS-BARMM Snapshot Survey, was designed and conducted across the region for this study, which is detailed in the following section.

\section{ALS-BARMM Snapshot Survey in 2018}

The ALS-BARMM Snapshot Survey was designed and undertaken to collect primary information on the status of ALS implementation across BARMM in 2018. All provinces except for Tawi-Tawi were covered and the fieldwork was undertaken between November and December 2018 and in February 2019. The World Bank (WB) partnered with the Consortium of Bangsamoro Civil Society (CBCS), a local nongovernment organization, to conduct the survey. CBCS established a survey team with enumerators who are native to the respective provinces and trained in the survey protocols. CBCS and WB supervised the training, field pilots, and actual interviews. Despite significant levels of security concerns and local conditions in the area, the survey was generally successful, as all preparations and coordination were satisfactory in the tight timeframe. DepEd Central Office and the former DepEd-ARMM Regional office provided the survey team with strategic direction and full support in coordinating with respective School Division Offices and individual officials ${ }^{5}$. The map below captures the location of ALS learning facilitators who participated in the survey (Figure 2). 
Figure 2: Map of the ALS-BARMM Snapshot Survey

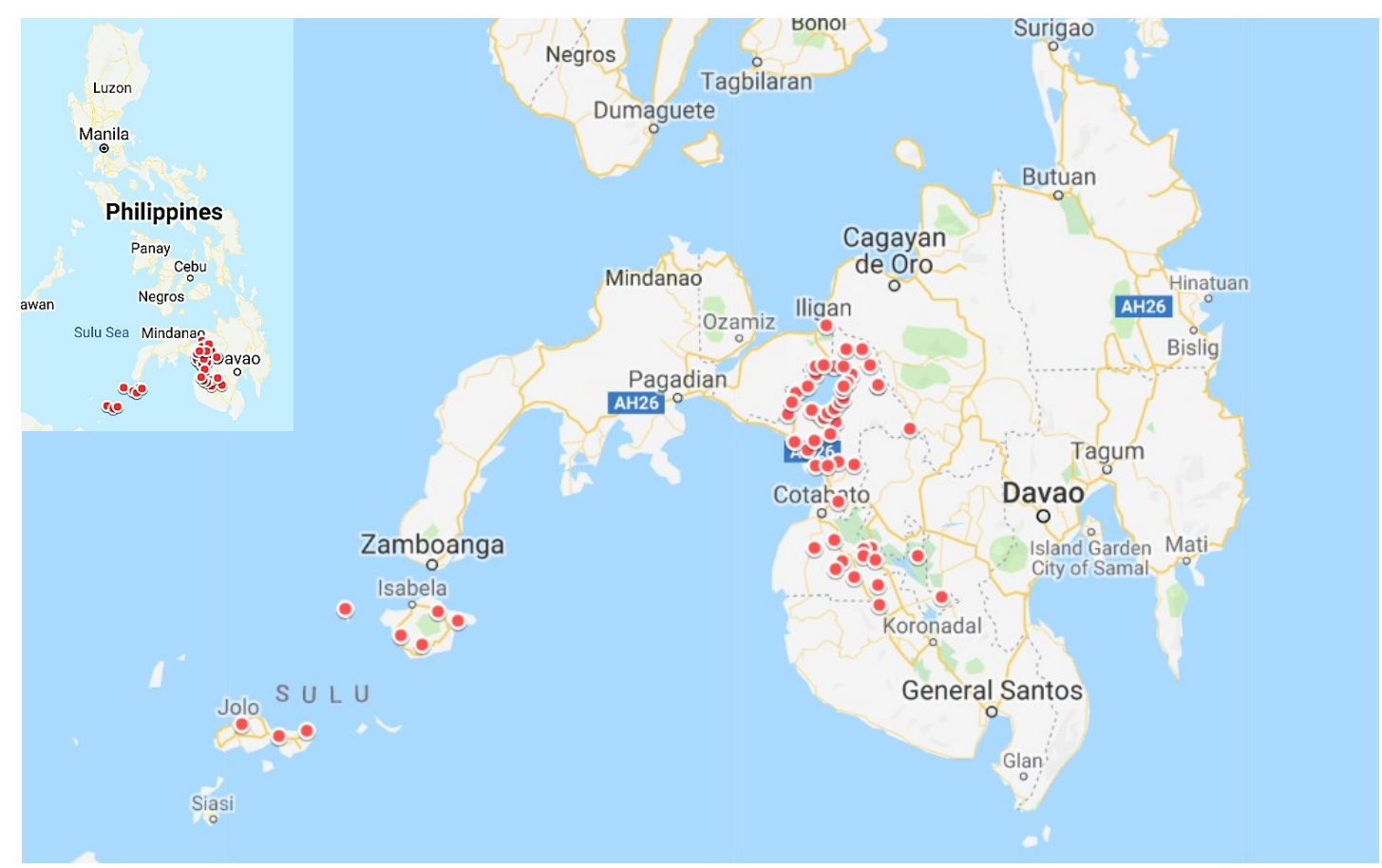

Source: ALS-BARMM Snapshot Survey 2018, World Bank.

The survey consisted of three modules: (a) detailed interviews with all ALS learning facilitators (LF) in BARMM, (b) a survey on the previous ALS enrollees of 2016 (or 2017) from randomly-selected facilitators, and (c) interviews and direct assessments with randomly-selected current ALS enrollees. The actual survey tools were modified and contextualized to BARMM using those used in the past WB studies by making questions sensitive to local cultures, ethnic groups, and religions and adding more questions about school dropouts and irregular practices while keeping them comparable to other national and regional benchmark data ${ }^{6}$. Additionally, this survey included short direct assessments on both functional literacy and socioemotional skills of ALS learners (See Box 2). Table 1 summarizes the actual survey samples.

Table 1: Summary of the ALS-BARMM Snapshot Survey Samples

\begin{tabular}{|c|c|c|c|c|}
\hline \multicolumn{2}{|c|}{$\begin{array}{l}\text { Province Cluster and School } \\
\text { Division Offices }\end{array}$} & \multirow{2}{*}{$\begin{array}{l}\text { Module 1 } \\
\text { Interview with ALS } \\
\text { LFs } \\
\\
8\end{array}$} & \multirow{2}{*}{$\begin{array}{l}\text { Module } 2 \\
\text { Survey on the previous } \\
\text { ALS learners with } \\
\text { randomly-selected LFs* } \\
\end{array}$} & \multirow{2}{*}{$\begin{array}{c}\text { Module } 3 \\
\text { Interview and direct } \\
\text { assessments with randomly- } \\
\text { selected current ALS } \\
\text { enrollees } \\
\end{array}$} \\
\hline Cluster 1 & Lamitan City & & & \\
\hline & Basilan & 16 & 5 & 99 \\
\hline \multirow[t]{3}{*}{ Cluster 2} & Marawi City & 13 & 4 & 74 \\
\hline & Lanao del Sur I & 52 & 6 & 115 \\
\hline & Lanao del Sur II & 48 & 7 & 105 \\
\hline \multirow[t]{2}{*}{ Cluster 3} & Maguindanao I & 27 & 6 & 117 \\
\hline & Maguindanao II & 18 & 5 & 93 \\
\hline Cluster 4* & Jolo $^{7}$ (Sulu) & 5 & 5 & 100 \\
\hline \multicolumn{2}{|r|}{ Total } & 187 & 42 & 785 \\
\hline
\end{tabular}

Source: ALS-BARMM Snapshot Survey 2018, World Bank. 
Note: *Shown the number of randomly-selected LFs. They were requested to report all enrollees or at least 75 in 2016 (or 2017 ). 75 is the minimum number of enrollees per LF, which is instructed by DepEd each year. The total number of past learners reported by these LFs was 3,724.

\section{Box 2: Direct Assessment of Skills with ALS Learners}

Years of schooling or level of education is an imperfect measure to express individuals' skills and learning, and there is no data to describe the skills profile of ALS learners. Thus, a direct assessment was designed and incorporated in the ALS-BARMM Snapshot Survey to understand both cognitive and socioemotional skills of ALS learners in BARMM. In order to analyze the skills relative to the national average, this direct assessment was adopted from the Functional Literacy, Education, and Mass Media Survey (FLEMMS) administered by the Philippine Statistic Authority (PSA) in 2013 and the STEP Skills Measurement Survey administered by World Bank in the Philippines in 2015.

First, FLEMMS is administered every five years to identify literacy skills with Filipinos between ages 10 and 64 (PSA 2013). Those questions and the survey micro data are publicly available. FLEMMS collects individual background information and includes those to assess basic literacy and numeracy and functional literacy such as problem solving and use of natural resources.

Second, socioemotional skills are adopted from the Skills Toward Employment and Productivity (STEP) program developed by WB to measure cognitive skills (reading, writing, and numeracy), socioemotional skills (personality, behavior, and preferences) and job-specific skills (subset of transversal skills with direct job relevance) of a representative sample of adults ages 15-64, whether they are employed or not. As the module of cognitive skills in STEP is not publicly available, the module on socioemotional skills is publicly accessible and used in the ALS-BARMM Snapshot Survey.

The survey was designed so that facilitators and participants were sampled proportionally to the size of operation in each school division. By strictly enforcing the survey protocol and carefully checking the data, some proxy or ghost teachers and learners were found concentrated in two small areas rather than scattered across BARMM. To ensure the reliability of reported answers, observations collected from these proxy/ghost facilitators and participants were removed from the final dataset for analysis. However, the research team admits that this made the actual distribution of data across divisions slightly uneven. Fortunately, the sample size was sufficiently large enough to accurately represent the situation in BARMM. However, this lack of reliable data such as a master lists of ALS facilitators is an issue that needs to be addressed by the BARMM Education Ministry (See more detail in Box 7).

\section{Sector Context}

\section{Basic Education Sector Context in BARMM}

The Philippines made remarkable progress in improving the quality of basic education in the past decades, demonstrated in various indicators, but at the same time, the system has faced many challenges. One of the major problems observed in the past decades is high dropout rates, especially in secondary education. Hence, the necessity of having a second-chance program to certify educational attainment outside the formal school system in the Philippines is closely associated with the formal school system and its challenge. A mirror image of the high prevalence of dropouts, now and past, is potentially the large number of people who wish to complete basic education, outside the formal school system, to increase their prospects and be productive in the economy.

Across all levels of the formal education, BARMM consistently had the lowest participation and completion rates among all regions ${ }^{8}$. In 2017, the Net Enrollment Rate (NER) in BARMM fell below the national average by about 20 percentage points at the elementary level and 45 percentage points at the JHS level (Figure 3). In BARMM, increasingly more students have been able to graduate from elementary 
schools; however, school completion rate for elementary schools was 54 percent against a national average of 94 percent in 2017. At the JHS level, around 62 percent of Grade 7 students continued to study until Grade 10, where was the national average was 85 percent (Figure 4). In BARMM, the dropout rate per grade worsened from 11 percent in 2010 to 14 percent in 2017, which is accumulated through a basic education cycle and has led to significantly low school completion.

In other terms, only 1 out of every 10 students who began primary education in BARMM would eventually graduate from lower secondary education on time. If there were 10 children living in BARMM, at best eight out of those 10 children would enter Grade 1 on time, but only five would complete Grade six. Then, two out of the four children who graduate from elementary school would enroll in JHS. Eventually only one child out of that 10-child cohort would complete Grade 10 on time. This leaves nine children for every 10 who are out of school without completing the full basic education cycle. Long-lasting conflicts and violence in BARMM and in its surrounding regions, have deprived children of the opportunity to study in school under stable conditions.

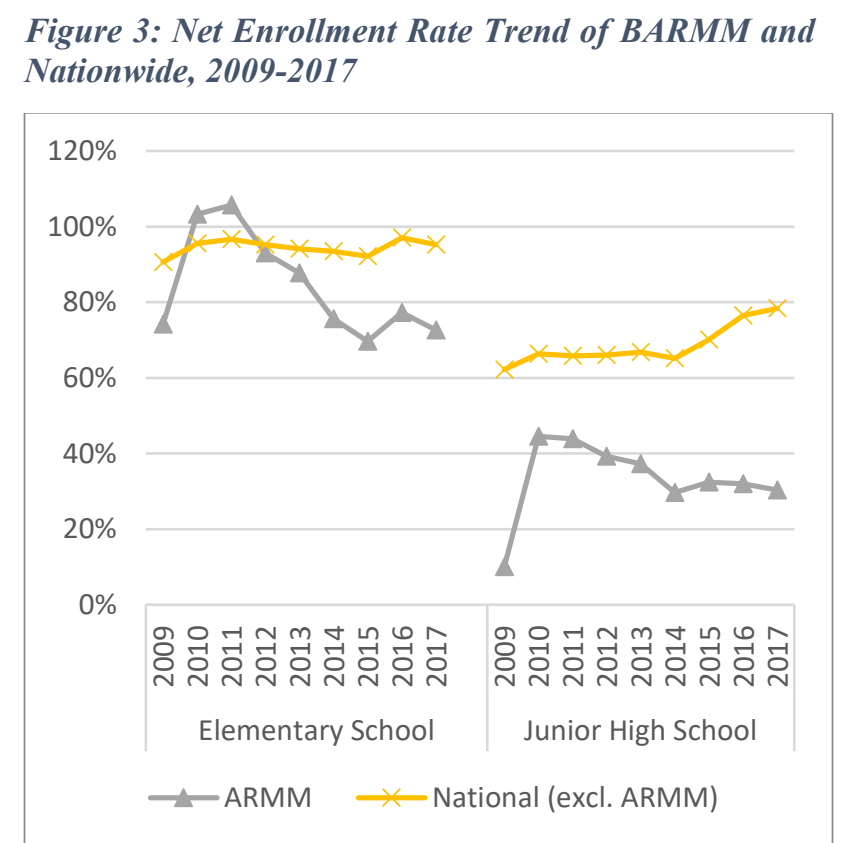

Source: EBIES, DepEd, 2009-2017.
Figure 4: School Completion Rate Trend of BARMM and Nationwide, 2009-2017

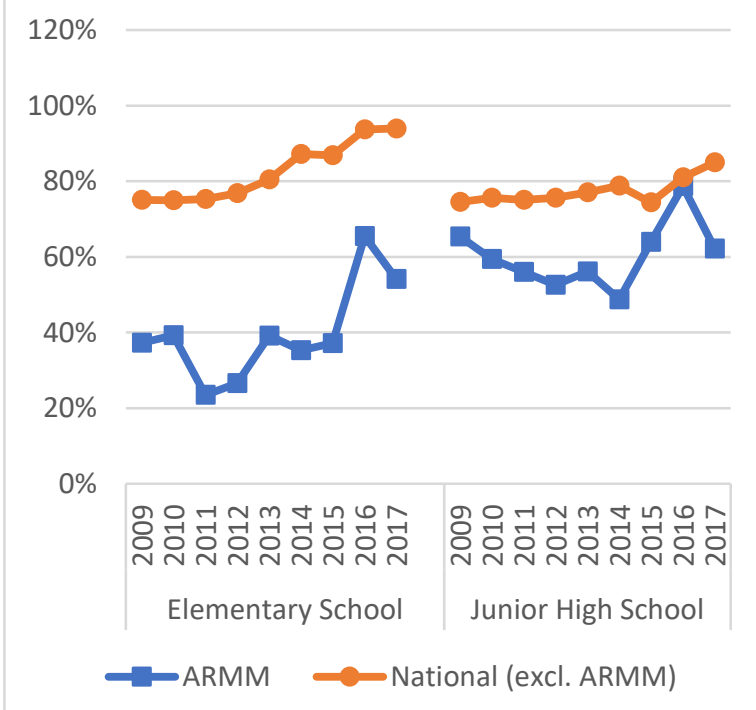

Source: EBIES, DepEd, 2009-2017.

These figures point to a crisis in the delivery of basic education in the BARMM area - a crisis which needs to be addressed directly to provide better quality education to its young people and to avoid further accumulation of the OSYA population. Complementary to the efforts for providing quality education for children in BARMM, ALS plays an important role to serve uneducated and low skilled youths and adults.

\section{ALS Sector Context in BARMM}

This section attempts to describe the ALS sector performance using common indicators such as participation/enrollment rate, completion rate, and A\&E passing rate, based on the DepEd's official records and national household survey data.

\section{Out-of-School Youth and Adults (OYSA)}

This section first aims to give a realistic estimate of prospective ALS learners in BARMM. First, we considered the upper age limit. The previous WB studies on ALS (Yamauchi et. al, 2016; Igarashi, 2018) 
show that the ALS programs are only appealing to youth and young adults, with close to 90 percent of learners concentrated between age 15 to 30 . This make sense as the opportunity costs for attending adult learning programs like ALS increase with age while net-gains for attaining an additional educational level would diminish with age. It will be unrealistic and inefficient if we only include those who did not complete basic education into ALS. The likelihood of participating in adult learning significantly varies by age.

This estimation excludes dropouts who have left schools within the past two years before completing the full basic education cycle. ${ }^{9}$ This is because they are considered to still be in transition and could, with remedial support, return to schools more easily relative to those who left school a long time ago. Also, overaged students are typical across the country as some of them delayed the start of schooling for various reasons and others repeated grades or temporarily suspended attendance. For example, school dropouts who are 16 years old are considered potential learners if the child is out of school before completing Grade 8 . Being older than grade-appropriate ages by three years or more significantly increases the chance of leaving school permanently. The main purpose here is to generate a figure that is close to the reality on the ground, but it is important to keep in mind that it is a variable depending upon the parameters for estimation.

The estimated size of the out-of-school population between ages 16 to 30 is around 6.5 million with 500,000 in BARMM (Table 2). The share to the total population in their age cohort is around 23 percent nationwide but is double (46 percent) in BARMM. For comparison, the estimated numbers of those who are out of school and below the official basic school completion age are also presented in Table 2.

Table 2: Summary of Out-of-School Population: Nationwide and in BARMM

\begin{tabular}{|c|c|c|c|c|}
\hline \multirow[b]{3}{*}{ Age group } & \multicolumn{4}{|c|}{ Out-of-school } \\
\hline & \multicolumn{2}{|c|}{ Nationwide } & \multicolumn{2}{|c|}{ BARMM only } \\
\hline & Number & $\%$ in age cohort & Number & $\begin{array}{c}\% \text { in age } \\
\text { cohort }\end{array}$ \\
\hline Elementary school age (6-11 years old) & 46,000 & $0.3 \%$ & 10,000 & $1.5 \%$ \\
\hline Junior-high school age (12-15 years old) & 299,000 & $3.2 \%$ & 50,000 & $12 \%$ \\
\hline Youth (16-24 years old) & $3,419,000$ & $19 \%$ & 276,000 & $38 \%$ \\
\hline Young adults (25-30 years old) & $3,157,000$ & $30 \%$ & 233,000 & $62 \%$ \\
\hline Total (16-30 years old) & $6,576,000$ & $23 \%$ & 509,000 & $46 \%$ \\
\hline
\end{tabular}

Source: Labor Force Survey, 2018, World Bank staff calculation.

Note: Share of people who are out of school and below respective age-appropriate school grade by more than 2 years.

Within BARMM, over half of the out-of-school population between age 16 and 30 are concentrated in Maguindanao and Lanao Del Sur provinces. Island provinces (Basilan, Sulu, Tawi-Tawi) have the remainder of the population. It is important to note that out-of-school youth and young adults in Maguindanao, Sulu, and Basilan outnumber those who are aged 16-30 and successfully completed basic education. The available data such as national household survey shows us spots of OSYA concentration, but limited efforts have been made to consider a targeting mechanism to allocate limited teaching positions to areas where OSYAs are concentrated.

Box 3: Practical Approaches to Quantify Prospective Beneficiaries for ALS

Once children are outside of the formal school system, it becomes difficult to locate them. Often, they are invisible, informal, and mobile. Understanding how many and where they are located is critical as that will help determine the scale of programs and target available resources more efficiently to those needed.

ALS facilitators annually conduct literacy mapping through house-to-house visits to list household members and identify who lacks basic education. From the mapping, facilitators develop microdata, which suggests potential learners for ALS. This mapping exercise can cover only selected communities, so it is not helpful 
for DepEd to grasp the total headcounts of out-of-school children, youth, and adults (OSCYA) across the country. Every year, two months are spent for this, so it will be unrealistic to anticipate increasing the coverage of the mapping data in the current format, though this community mapping is an effective exercise for advocacy and outreach.

First, we have proposed a practical approach is to estimate using household survey data collected by PSA (Yamauchi et.al 2016; Igarashi 2018; and David and Albert, 2015). The Annual Poverty Indicators Survey (APIS), Labor Force Survey (LFS), and other nationwide surveys provide reliable estimates of socioeconomic and demographic states at the national and regional levels. Benefits of using national household surveys are that we can analyze the trend over time and compute the estimate without much time lags.

Second, if it is necessary to determine the number of OSYA within smaller geographic boundaries, the Listahanan data (the National Household Targeting System for Poverty Reduction) can be used. It is a very comprehensive database collected by staff of the Department of Social Welfare Development (DSWD) through house-to-house visitation every three years across the country and provides the actual headcount of out-of-school population at the barangay level (smallest administrative unit) and municipality/city levels (Velarde, 2018). It also collects basic characteristics of individuals including gender, age, schooling status, etc. The headcount data should be helpful for field implementers. The latest available data was collected in 2015 .

Both approaches should provide similar figures of OSYA (Figure 5). There are noticeable differences, particularly in urban regions (such as Region 4, Region 3, NCR, and Region 7) while non-urban regions provide almost identical figures except BARMM. This is because Listahanan includes all rural areas and only pockets of poverty in urban areas.

It is important to choose a suitable approach depending on specific purposes. If it is important to generate figures to show the demand for ALS at the national or region levels overtime, it makes sense to use national household survey data (possibly mixing different national household surveys such as LFS, APIS, and FLEMMS for triangulation). If setting targets in smaller jurisdictions, the Listahanan data will be a very useful source. A key challenge is that the Listahanan data, national household survey data, and DepEd's learner information system are all independent systems, but it will be powerful if these programs could be shared or interlinked.

Figure 5: Comparison of the Number of OSYA (below age 30) Using LFS and Listahanan

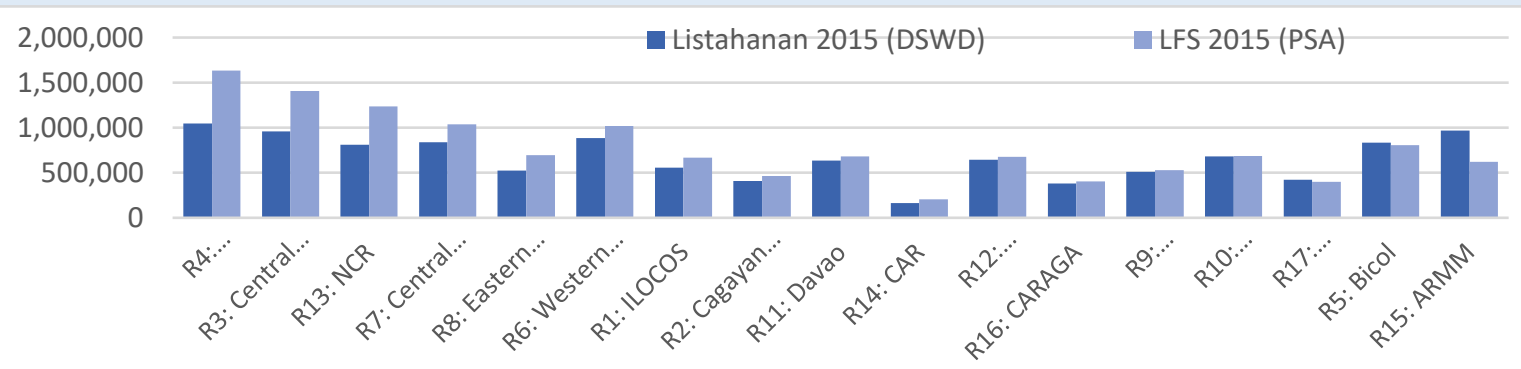

Source: LFS 2018, PSA; and Listahanan 2015, DSWD.

\section{Out-of-School Children}

Based on the national household survey data, the number of young children (ages 6-11) who are not in school and below respective age-appropriate grade is estimated around 49,000 across BARMM despite laws requiring their attendance. A delicate question remains whether to enroll children who are already outside of the formal school system in ALS, given poor access to schools in BARMM. 
There are three different approaches to addressing the needs of this population. The first approach is finding ways to get these children back into regular schools and learn with their peers. This is the most effective educational approach for these children, even while presenting challenges for teachers and schools. DepEd also operates an Alternative Delivery Mode (ADM), which provides school-based interventions to support students who are at risk of dropping out by offering flexible learning modalities while they are still in school. ADM follows the K-12 basic education curriculum, which is structured distinctly from the ALS curriculum. Providing remedial support to younger out-of-school children (who are within the official school age) by school teachers may be a more desirable option.

However, the scale of the ADM operation is very small across the country and has not been fully rolled out across BARMM. Its expansion is one of the priorities for the new government as stated in the BARMM Alternative Learning Modalities (ALM) Five-Year Roadmap. ADM programs had approximately 29,000 students comprised of 6,000 at the elementary level and 23,000 at the secondary level across all regions in 2016 (DepEd). Moreover, there is a significant lack of regular schools accessible to all the schoolage population of BARMM. BARMM still has over 250 barangays which do not have any public elementary schools in 2018 (DFAT 2017; DFAT 2019).

The second approach, in conflict-affected areas that do not have public schools, is for the government partnership with other partners through a contractual or voucher mechanism. In BARMM, the Australian government funded the BEAM project which piloted ADM in ARMM in 2012 with an NGO, which shows that, in the conflict-affected areas, this model has been demonstrated as means to reach out to areas which do not have public schools.

Given the reality of the educational landscape, a third approach has emerged which is for children of school age to enroll in ALS, especially in the A\&E program. As noted, this is the least satisfactory option but is a reality with which the ALS in BARMM will have to cope for some time. Many children in BARMM, who were in the extreme condition and deprived of studying in the formal school education for the reasons such as conflicts and violence, should deserve "second-best" alternative learning modalities as a complementary pathway for continuing and completing their schooling.

It is extremely critical to harmonize efforts to improve the formal school-based education, ALS, ADM, and other education programs holistically for BARMM at the policy and local levels. The new BARMM ALM Five-Year Roadmap providing a holistic action plans with concrete indicators for implementation and monitoring will be an important guide for the new government to respond to the educational needs of out-of-school children, youth, and adults.

\section{Participation rate in ALS}

Unlike the formal school enrollment rate, computation of ALS participation rates (PR), or enrollment/coverage rates over OSYA is complex. ALS accepts learners of all ages in principle, and the age distribution of learners is very wide. However, if a PR is estimated by using Filipinos of all ages who have less than required basic education for a denominator, the estimated PR will be extremely small. Rounds of the past ALS surveys revealed that ALS programs are predominantly appealing to the younger population who are under age 30 particularly. For this reason, also for simplicity and objectiveness, we use the age restriction in defining the denominators. For numerators, we use the number of ALS participants extracted from the LIS of DepEd.

Across the country, the number of national enrollers in ALS has increased seven-fold, between 2005 and 2018 from 106,000 to 840,521 across the country (Figure 6). Overall, the program has seen a rapid 
increase in both enrollment and completion of the program, particularly accelerated since the Duterte Administration.

Figure 6: Trends in ALS Performance, Nationwide

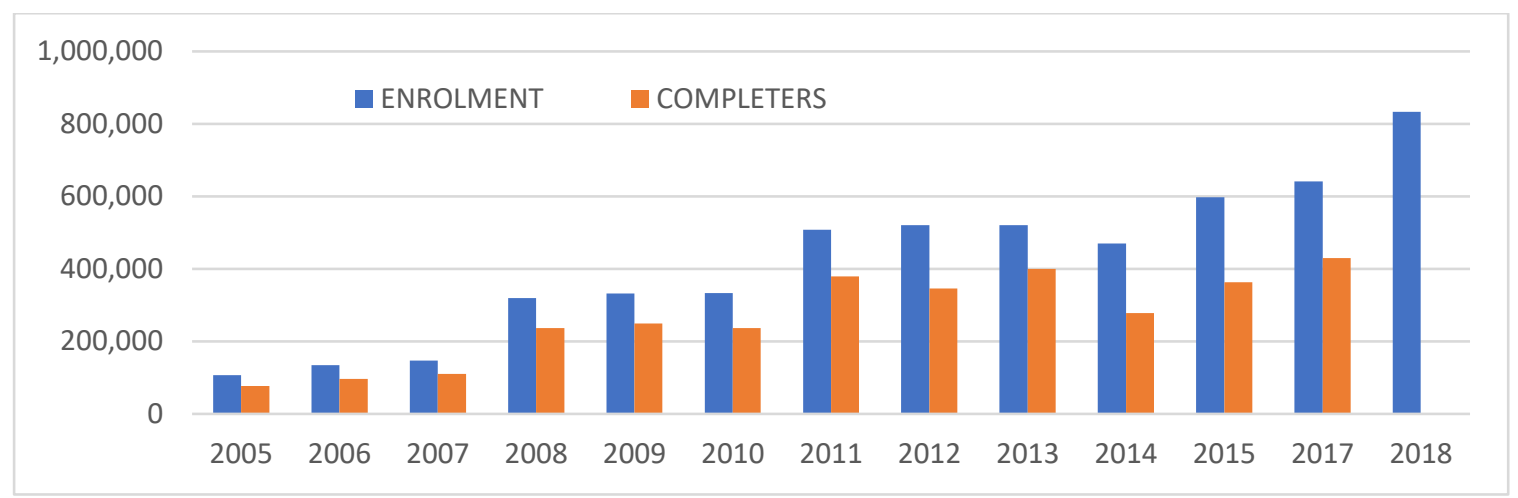

Source: DepEd BALS, LIS data, 2005-2018.

In BARMM, the number of ALS learners also grew at an even faster rate from 2,369 to 25,131 during the same period. The program has seen a significant increase over 12 years, but annual ALS enrollment in BARMM fluctuated significantly from year to year in the past, suggesting certain challenges in the official data. ${ }^{10}$ The enrollment data is now generated by automatically consolidating individual ALS learners registered in the LIS, which contributed to the increased reliability of the official data in recent years.

Based on the approach described above, the current ALS participation rate is estimated around 13 percent nationwide, but it is only around 5 percent in BARMM (Table 3). Clearly, the region is seriously lagging while it has the largest share of OSYAs. It is unrealistic to anticipate a substantial increase such as near-full coverage given the magnitude of school dropouts in BARMM. The 2018 OECD report suggests that only about 20 percent of adults who have lower skills participated in adult learning programs, ${ }^{11}$ while adults with medium and high skills participate more. ${ }^{12}$ It is important to set realistic targets of participation rates for ALS, which should be helpful in programing a sensible investment program or allocating resources more efficiently.

Table 3: Estimated OSYA, ALS Participants, and ALS Participation Rate

\begin{tabular}{|l|c|c|c|c|}
\hline & \multicolumn{2}{|c|}{ Nationwide } & \multicolumn{2}{c|}{ BARMM } \\
\hline & Number & (\% female) & Number & (\% female) \\
\hline OSYA Aged 16-30 (LFS 2018) & 6,58 million & $38 \%$ & 0.51 million & $48 \%$ \\
\hline ALS Participants (DepEd 2018) & 840,521 & $45 \%$ & 25,131 & $60 \%$ \\
\hline Participation Rate in ALS & \multicolumn{2}{|c|}{$13 \%$} & \multicolumn{2}{c|}{$5 \%$} \\
\hline
\end{tabular}

Source: Labor Force Survey (2018), DepEd LIS data (2018). WB staff calculation.

\section{Completion rate in ALS}

While around 65-70 percent of those enrolled in ALS complete their learning across the country, the completion rate in BARMM is around 35 percent. Among all of the ALS programs, the completion rates of the A\&E Elementary and Secondary level programs in BARMM are about half of the national level (Table 4). Completion is determined based on whether learners have completed their own individual learning agreement (ILA), which are established at the time of enrollment through recognizing their prior learning (RPL), skills and competencies during program registration. In BARMM, as well as other regions, 
there are some cases, in which the ILA was not developed nor was RPL undertaken. A clear example of non-completion is when learners stop attending sessions and thus miss being assessed at the end of the interventions.

Table 4: ALS Enrollment, Completion, and Completion Rate of BARMM and National Average

\begin{tabular}{|c|l|r|r|}
\hline ALS Program & No. of learners & Nationwide & BARMM \\
\hline \multirow{3}{*}{ BLP } & Enrolled & 57,623 & 8,427 \\
\cline { 2 - 4 } & Completed & 32,358 & 3,375 \\
\cline { 2 - 4 } & \% completed & $\mathbf{5 6 \%}$ & $\mathbf{4 0 \%}$ \\
\hline \multirow{3}{*}{ A\&E Elementary } & Enrolled & 107,876 & 4,108 \\
\cline { 2 - 4 } & Completed & 65,302 & 1,143 \\
\cline { 2 - 4 } & \% completed & $\mathbf{6 1 \%}$ & $\mathbf{2 8 \%}$ \\
\hline \multirow{3}{*}{ A\&E Secondary } & Enrolled & 476,098 & 5,030 \\
\cline { 2 - 4 } & Completed & 332,698 & 1,870 \\
\cline { 2 - 4 } & \% completed & $\mathbf{7 0 \%}$ & $\mathbf{3 7 \%}$ \\
\hline \hline \multirow{3}{*}{ ALS Total } & Enrolled & 641,597 & 17,565 \\
\cline { 2 - 4 } & Completed & 430,358 & 6,388 \\
\cline { 2 - 4 } & \% completed & $\mathbf{6 7 \%}$ & $\mathbf{3 6 \%}$ \\
\hline
\end{tabular}

Source: LIS 2017; DepEd.

\section{A\&E Certification rate}

The ratio of those who passed the $A \& E$ exam to the total number of test takers remains significantly lower in BARMM than the national average. In 2018, BARMM's A\&E passing rates for the A\&E Elementary or Secondary level was behind the national average by only 20 or 24 percentage points, respectively. The A\&E passing rate was significantly high for 2018. While the number of the A\&E passers in BARMM continued to grow marginally between 2016 and 2018, the A\&E takers continued declining to a half during the same period even though the total number of ALS learners has been rising quickly.

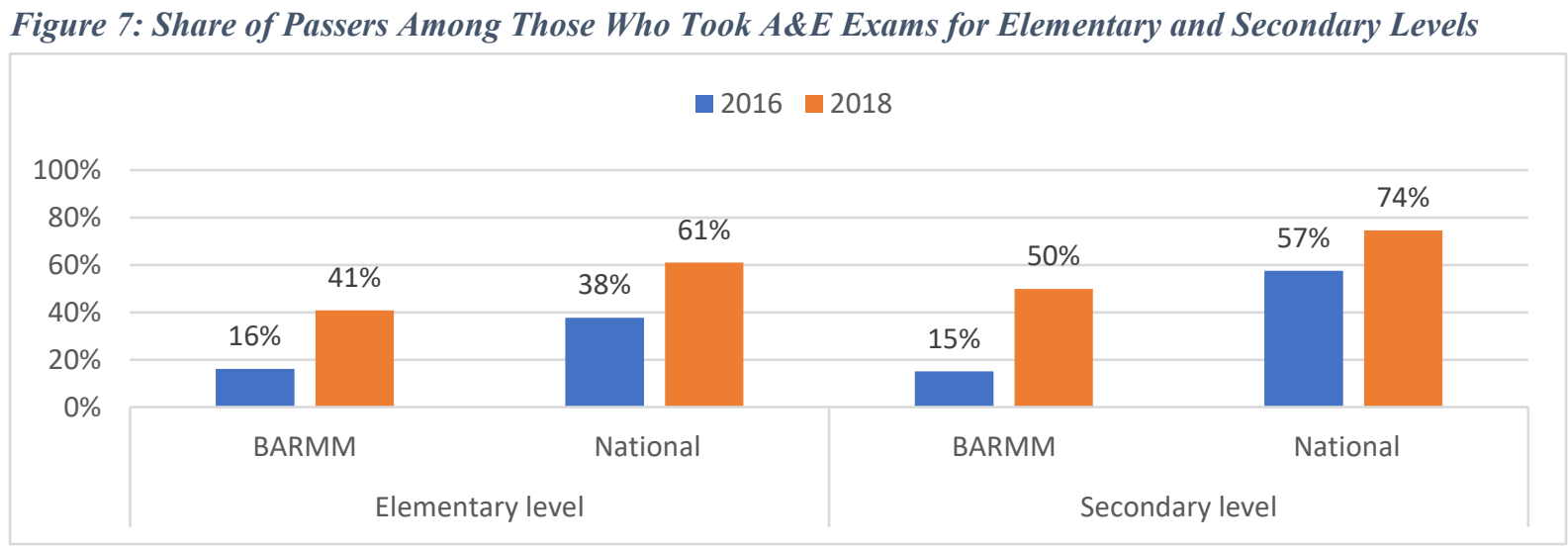

Source: A\&E test results, 2016 and 2018, Bureau of Educational Assessment, DepEd.

The number of $A \& E$ test takers significantly decreased in 2017 and 2018 across the country. The average number of the A\&E test takers between 2011 and 2016 maintained between 180,000 and 220,000 takers per year, but in 2017 and 2018, the numbers dropped to 82,000 and 64,000, respectively. In these years, the A\&E exam was not administered in the regular cycle but delayed due to challenges in procurement of the exam administration. This led to situations where ALS learners could not take the exam. Infrequent and delayed administration of the A\&E test results in huge wastage of investment in ALS learning programs, plus loss of opportunities for career growth and demoralization/demotivation. This 
suggests needs that A\&E tests should be held more frequently, in more accessible locations/modalities so that learners can complete it shortly after end of their ALS learning program.

More thorough data analysis will be necessary to understand ALS learners' performance who opted for equivalent credentials. The data also included those who did not participate in ALS but took the test, called walk-ins, as well as those who attempted the A\&E exam more than once. But unfortunately, the available data does not provide disaggregate information of the test takers in BARMM to do further analysis.

In sum, the available official data from DepEd points to significant challenges, showing that the quality of the ALS programs delivery seems below the national average in BARMM overall. Given the magnitude of OSYA, ALS has been and will remain highly significant in the region for the next few decades. ALS coverage over the OSYA seems limited to a very low level. Also, lower completion and A\&E passing rates may point to the significant quality challenge in the region. In the subsequent sections, we report the results of the 2018 ALS BARMM Snapshot Survey on both the demand and supply sides.

\section{Learners}

This section presents findings on the ALS learners from the 2018 ALS-BARMM Snapshot Survey to discuss their basic profiles, time commitment, skills, and motivations for learning in ALS.

\section{Basic Profiles of Learners}

\section{Background}

Among ALS programs, Accreditation and Equivalency (A\&E) programs are particularly appealing to youth and young adults in BARMM while basic literacy program (BLP) is attended by more elderly learners (Figure 8). However, the composition of these programs is different for BARMM. For example, more than half of learners are attending BLP or A\&E Elementary program, which includes only about one-quarter in other regions. BLP is attractive to older generations, particularly females, who might have missed education (Figure 9).

Figure 8: Share of Learners by ALS Program, BARMM (Inner ring), and Nationwide (Outer ring)

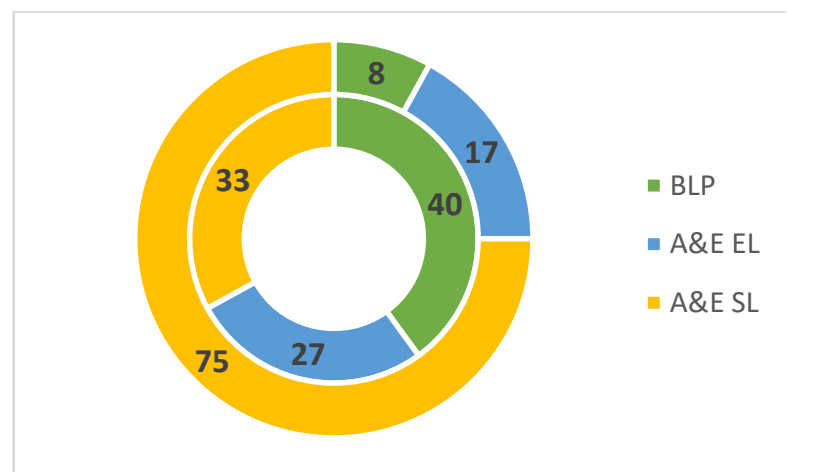

Figure 9: Age of Learners by ALS Program and Gender

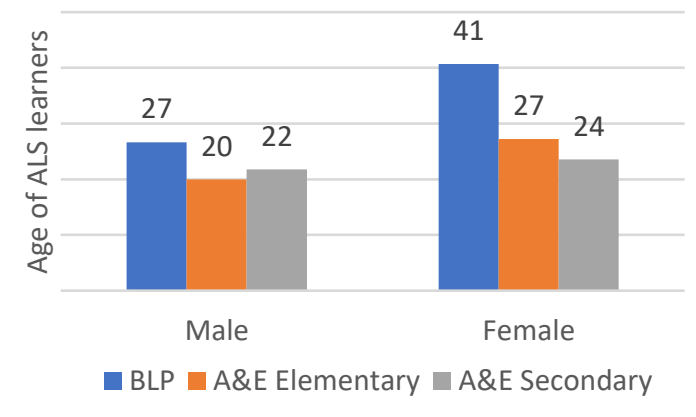

Source: DepEd LIS Data (2018); ALS-BARMM Snapshot Survey, 2018 World Bank.

Note: Outer ring represents nationwide, inner ring represents BARMM.

Profiles of ALS learners in BARMM somewhat differ from the profiles of learners nationwide. Across the country, the clear majority of ALS learners are youth and young adults, but their ages are distributed more widely in BARMM. Two-thirds of ALS learners in BARMM are female learners, which is not consistent with the average gender ratio of OSYA in other regions. Also, another contrast can be found in 
the schooling history. More learners in BARMM have never enrolled in formal elementary schools before, while learners in other regions had at least enrolled in elementary schools. Also, only one-third of learners in BARMM are working at the time of ALS enrollment, which is significantly lower than other areas.

Table 5: Profiles of ALS Learner Sample in BARMM

\begin{tabular}{|c|c|c|c|c|}
\hline \multirow[b]{3}{*}{ Question Criteria } & \multirow[b]{2}{*}{$\begin{array}{l}\text { Snapshot } \\
\text { BARMM }\end{array}$} & \multicolumn{3}{|c|}{ Benchmarks } \\
\hline & & Nationwide & Nationwide & $\begin{array}{l}\text { Great Metro } \\
\text { Manila }\end{array}$ \\
\hline & 2018 & 2015 & 2017 & 2013 \\
\hline Age (mean) & 28 & 28 & 23 & 24 \\
\hline Age (median) & 24 & 23 & 21 & 21 \\
\hline Share of female & $66 \%$ & $47 \%$ & $44 \%$ & $43 \%$ \\
\hline $\begin{array}{l}\text { Participants who ever enrolled in the formal elementary } \\
\text { schools. }\end{array}$ & $88 \%$ & $96 \%$ & $99 \%$ & $99 \%$ \\
\hline Participants who worked for pay when enrolled in ALS & $38 \%$ & $47 \%$ & $55 \%$ & $86 \%$ \\
\hline
\end{tabular}

Sources: National ALS survey, 2015, DepEd/WB; National Snapshot, 2017, WB; NCR-plus survey, 2013.

Note: * includes selected region/province across the country.

ALS learners who are married, particularly female learners, tend to be much older, suggesting significant opportunity costs for participating in ALS programs. The average age is just around 20 years old for both male and female learners who were not yet married while the distribution of learners' age is wider and gradually peaked around 35 years old (Figure 10). By marital status, there is a 15-year gap in the enrollment age of learners. As also seen in Table 5, over 65 percent of ALS learners in BARMM are female despite the fact that OSYA are mostly males. Many female learners are married at enrollment and defer enrollment in ALS programs for housekeeping and childcare (presumably until their children start attending schools) (Figure 11). On the other hand, the majority of male learners are not married.

\section{Figure 10: Distribution of ALS Learners by Age and Marital Figure 11: Marital Status of Learners by Status Gender}
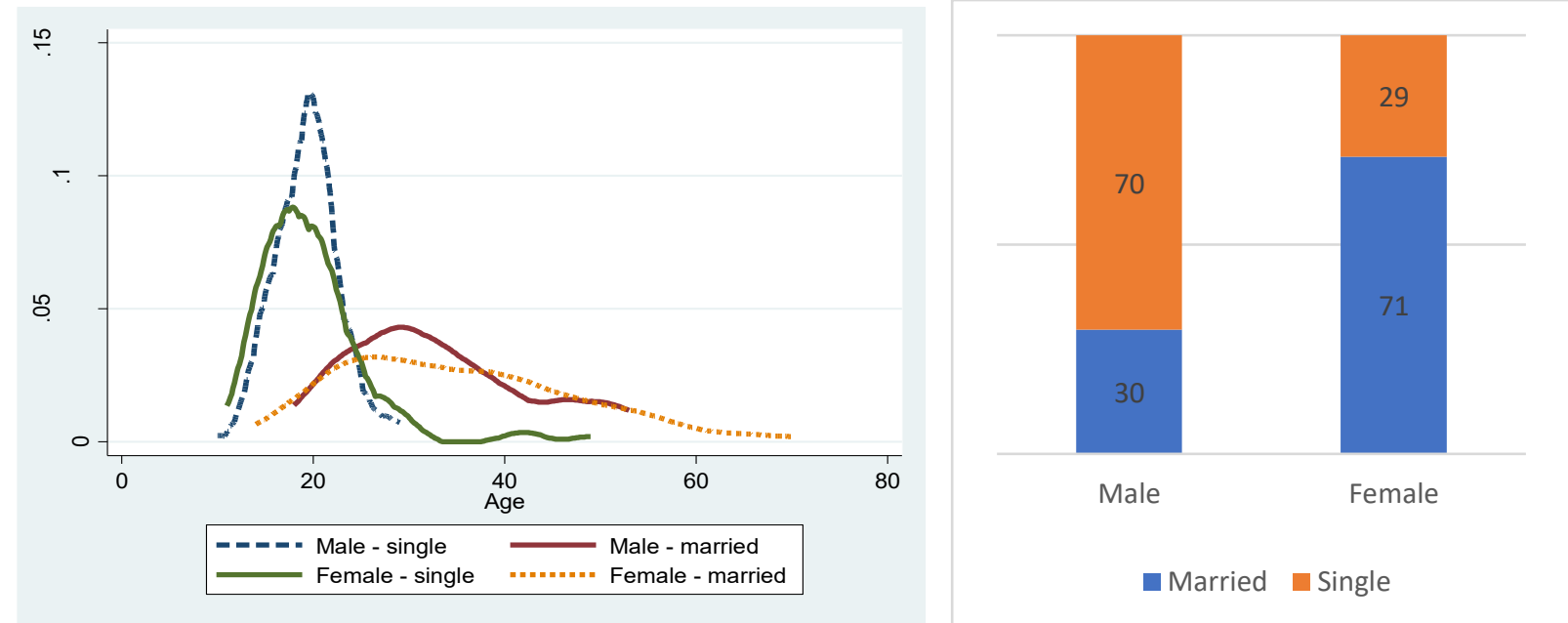

Source: ALS-BARMM Snapshot Survey 2018; World Bank.

Many younger ALS learners have completed elementary school, while there are many older and female learners who have no grades completed. For younger ones, over 60 percent of both female and male learners completed elementary school but did not enroll in JHS or were former JHS dropouts (Figure 12). In contrast, there were close to 40 percent of female learners who are above 30 years old who did not even complete the first grade in the formal school. They reported that financial difficulties of the family 
and limited understanding/support from family in attending school as the two top reasons why they never attended schools.

Figure 12: Highest Educational Level Completed Before ALS for Younger and Older ALS Learners (\%)
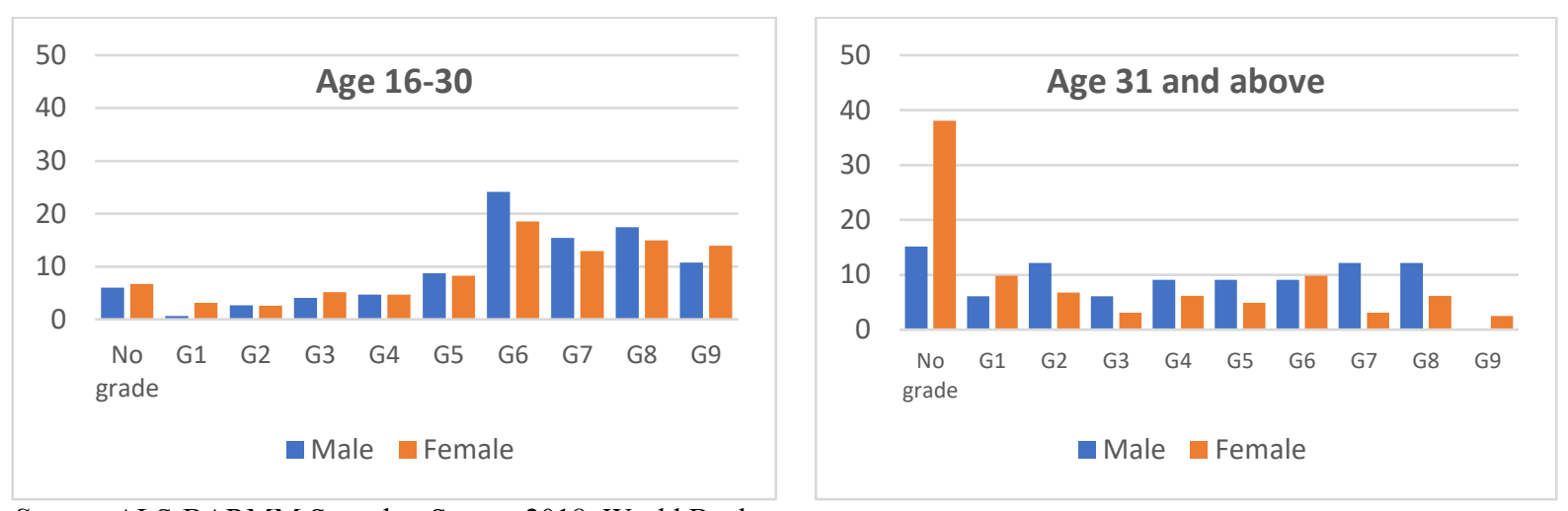

Source: ALS-BARMM Snapshot Survey 2018, World Bank.

\section{Dropout and Transition to ALS}

Consistent to other regions, more than half cited the financial difficulty of families in BARMM as the reason for dropping out or never enrolling in schools. The survey shows that 43 percent of ALS learners in BARMM said their families are beneficiaries of the Conditional Cash Transfer (CCT/4Ps) program. Access to school, including distance and security concerns, was the second most common reason for not attending school as reported by one-third of ALS learners. Particularly, a significant number of female learners reported that lack of support or understanding of family, as well as early marriage, in continuing schooling was the primary reason for not completing their education (Figure 13).

Also, efforts by schools to prevent and follow up with dropouts are rather limited. Only one-third of ALS learners talked to teachers or other school staff about their plans or upcoming situation of dropping out from school. However, 60 percent of ALS learners said that they just left schools without informing any school personnel. Also, two-thirds of ALS learners were never contacted by teachers or school staff after they stopped attending schools. Fortunately, at least half of all learners reported that they had some advice from community members after they dropped out (Figure 14), pointing to the potential role of the community as an advocate for schooling and ALS.

According to the learners, financial support and/or support to convince their parents might have helped them stay in school. Seventy percent of ALS learners cited financial support as one factor that could have helped them stay in school; 40 percent reported that they could have stayed in school if there were interventions to convince their own parents to send them to school. About 15 percent of ALS learners said that their teachers' support could have helped them to stay in school. While 40 percent of ALS learners in BARMM are from CCT beneficiary families, more than half are from large families (with at least five siblings) and still face significant financial difficulty. Expansion of CCT for ALS learners in BARMM could not only alleviate the flow of school children leaving schools, but also offset opportunity cost of joining in the ALS program among out-of-school secondary-level children. 
Figure 13: Reasons for Dropping Out of School

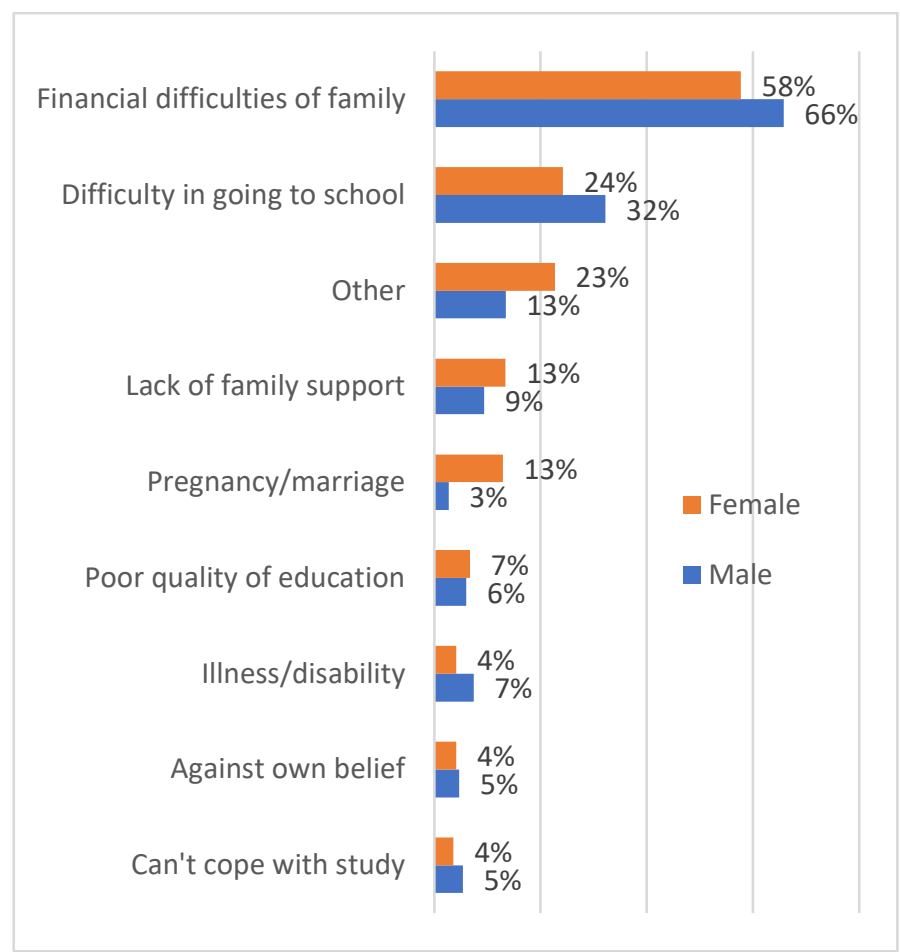

Figure 14: Share of Learners Considering Dropping Out

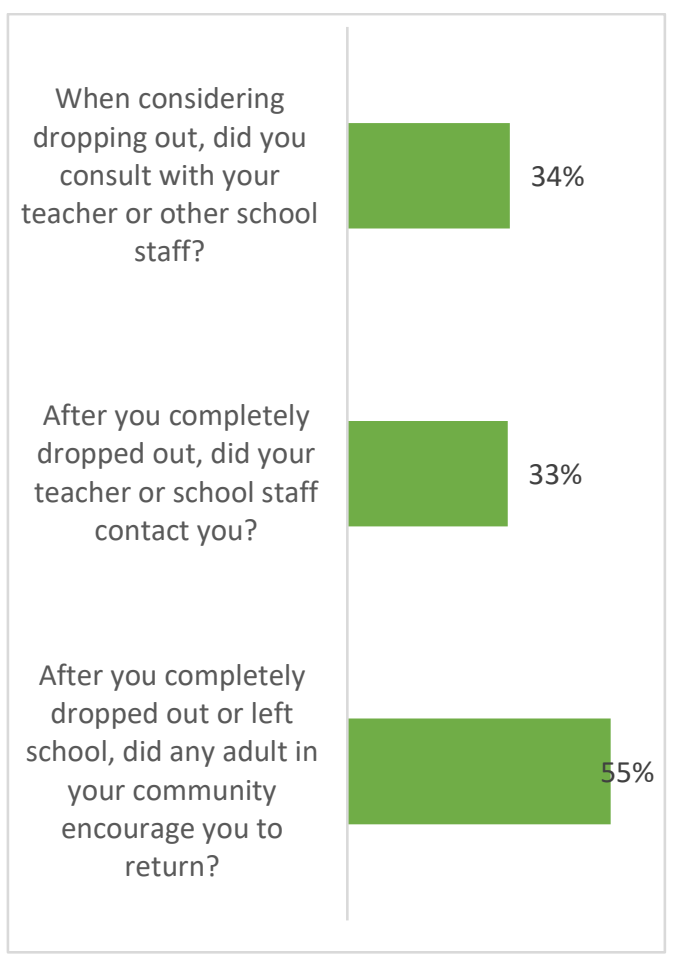

Source: ALS-BARMM Snapshot Survey 2018, World Bank.

Note: The graph shows shares of the respondents who reported that each of these conditions is either of the two most significant dropout reasons, and thus the numbers add up to more than 100 percent.

About 30 percent of ALS learners reported that they learned about ALS through ALS facilitators. A similar number also reported that school teachers were the primary ALS referral mechanism. This suggests that schools and ALS facilitators play important roles in advocating ALS to prospective learners. Also, twenty five percent of OSYAs were referred to ALS by family, friends or community members which is a significant number. In contrast, only two percent of learners reported learning about ALS through flyers, printed information, and media (internet, radio, TV). This is consistent across different profiles of learners. Furthermore, about 10 percent of learners in Maguindanao II Division reported that Barangay officials were their informants for ALS, which was not observed in other divisions in BARMM. This suggests that there may be strong partnerships between ALS, schools, and Barangay for reaching out to OSYAs, which may not yet be so common in other areas.

Motivations to enroll in ALS vary across programs. Over 70 percent of BLP learners primarily aim to acquire basic literacy (able to read, write, and do simple math) while less than 10 percent aimed for further education or training. The A\&E Elementary-level learners have more diverse motivations. About a quarter were interested in acquiring basic literacy skills and another quarter in gaining Equivalency at the elementary education level. About 30 percent wanted to pursue post-secondary or tertiary education credentials. The clear majority of A\&E Secondary-level learners were predominantly interested in achieving a college level or other educational credentials such as post-secondary vocational training or high school diplomas. Consistently across the programs, a small number of learners were motivated by a sense of belongingness, confidence, dignity, and so on. 
Figure 15: Reasons for Enrolling by ALS Program

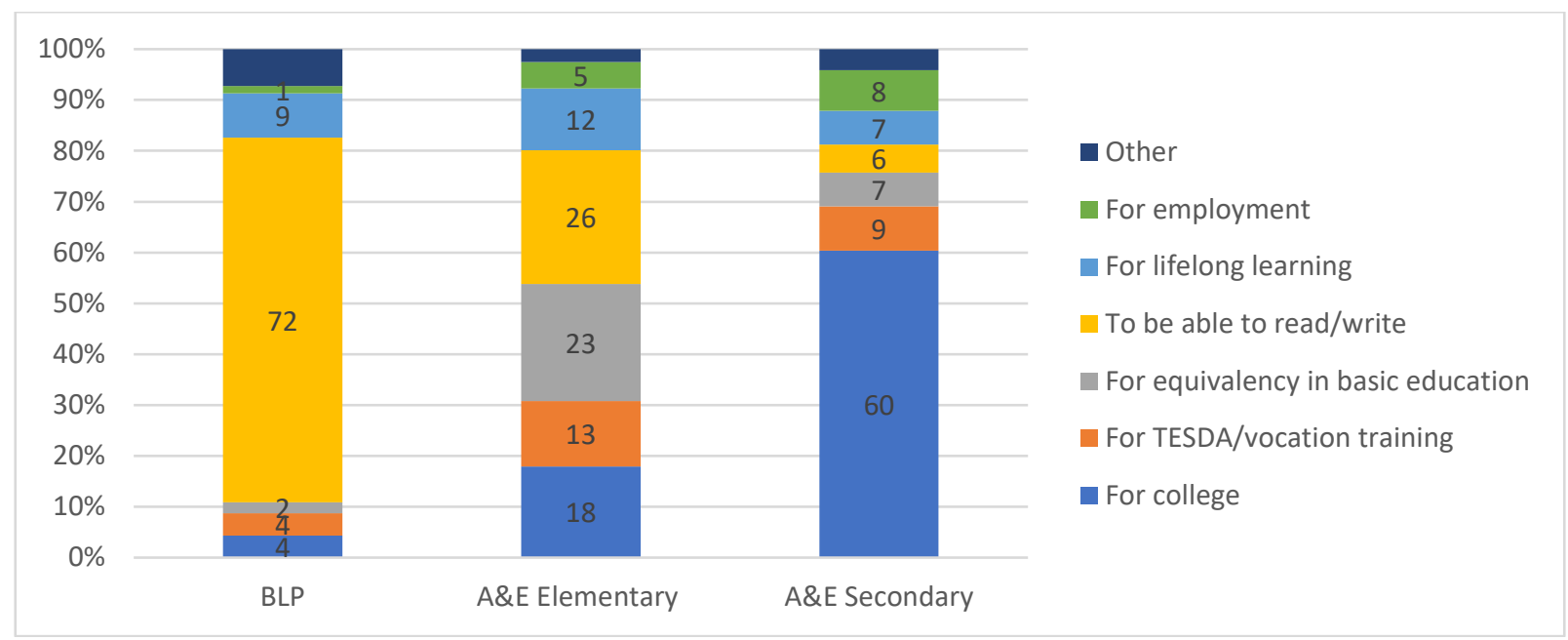

Source: ALS-BARMM Snapshot Survey 2018, World Bank.

\section{Learning in ALS}

Once enrolled, ALS learners in BARMM attend three sessions for a total of 10 hours per week, which is consistent across BLP, A\&E EL and A\&E SL programs. Besides, learners in A\&E programs spend about three hours per week in self-study such as homework and reading. Additionally, this is less than two hours per week among BLP learners. Also, the average commuting time to CLCs is one hour for one way either by walking ( 70 percent) or tricycles ( 20 percent). Learners devote about 20 hours per week to learning activities upon enrollment.

However, performance of ALS learners in BARMM are not at par with learners in other regions. The survey asked randomly-selected learning facilitators to report basic information on learners in 2016 and 2017 (Figure 16). The BARMM attendance and completion rates are lower by about 10 percentage points compared to the national average. The disparity between BARMM and other regions is significant on the share of A\&E passers to ALS learners. ${ }^{13}$ However, caution is needed in interpreting this as it could be attributable to the fact that ALS learners in BARMM could not perform well on the test and/or that higher share of BLP and A\&E elementary learners who are much less likely to sit in and pass the A\&E exam affected the computation. BLP learners will not take the A\&E test since they are not ready to do so, and it is not part of the program design. Due to the limitation of the collected data, the figure is showing the share of A\&E test takers and passers to the overall ALS learners in BARMM. (See Box 4)

Figure 16: Share of ALS Learners Who Attended, Completed, Took the A\&E Test and Passed

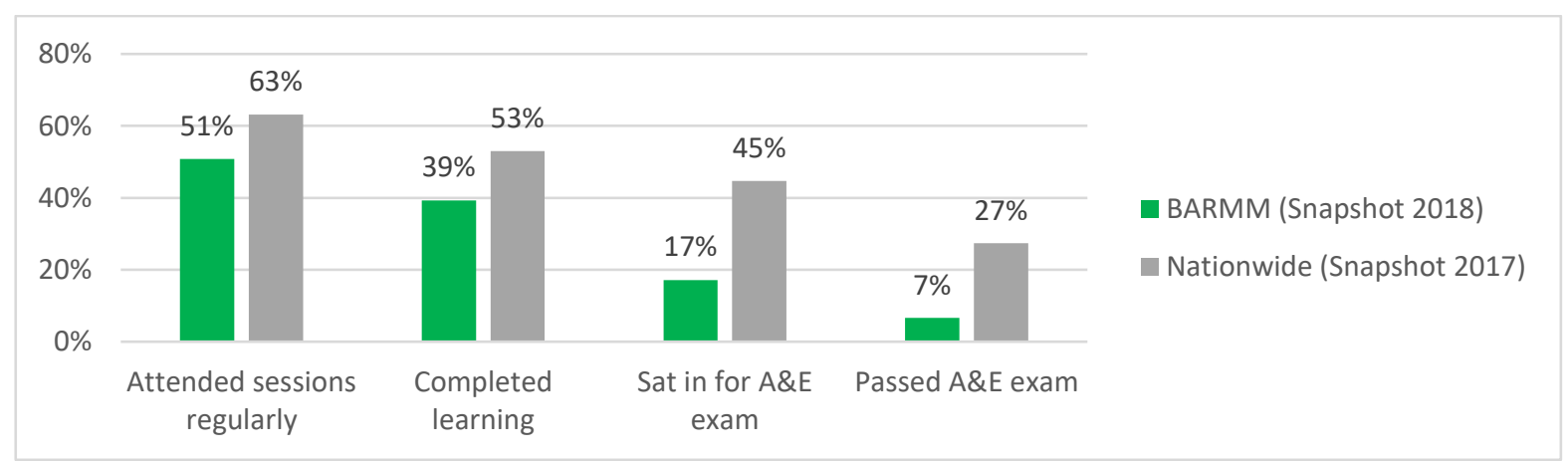

Source: ALS-BARMM Snapshot Survey 2018, World Bank. 
Box 4: Snapshot Survey as a Cost-efficient Approach

There have not been standardized practices to record ALS learners' performance and activities after completing the ALS programs. In addition, A\&E exam results were not reported timely or cross-linked to the implementation data. Thus, it is difficult to discuss the benefits of the program for learners in an objective way though there is a mass of anecdotal evidence about successful ALS graduates.

A tracking survey was designed and conducted for both ALS learners and non-learners. After several years, they were initially recorded through community mapping (Yamauchi et. al, 2016). The study generated objective evidence on labor market outcomes by ALS program. ALS learners have higher earnings and employment probability if they passed the A\&E test compared to the OSYA who did not go through the ALS program. The tracking survey was an effective means to understand the post-ALS routes of the learners but also an expensive, difficult, and time-consuming exercise.

In the follow-up, a simplified approach called the Snapshot Survey was designed and undertaken based on the self-reporting by ALS facilitators who were randomly selected in 2017 in specific areas across the country, as well as in this study in BARMM. In this approach, ALS facilitators who were randomly selected were asked to report basic information of all of their learners registered in the Learner Information System (DepEd database) two to three years preceding the Snapshot. As each facilitator is required to have at least 75 learners each year to pay for operational allowances, asking facilitators to report all 75 learners per year during the survey is critical in minimizing the bias in the self-reporting survey. The primary data was carefully reviewed and validated by a survey group. In addition, random spot-checking with past learners was performed to test reliability of the information reported.

Although this is not a rigorous approach compared to the tracking survey, the data from Snapshot Surveys is adequate in generating over-time trends of performance of the program, especially whether ALS learners benefit from going through the ALS intervention or not.

\section{Skills of Learners}

This study also aimed to assess the academic (cognitive) and socioemotional (non-cognitive) skills of ALS learners in BARMM. The performance indicators that are always used to describe the ALS program (e.g. previous section) implementation are useful but provide limited dimensions of ALS learners' skills. In this study, we incorporated two simple modules to assess academic skills (specifically, functional literacy) and socioemotional skills such as soft or behavioral skills (See Box 5). Note that the measured skills are not attributable to the impact of ALS program, but merely provide objective information about ALS learners.

\section{Functional Literacy Skills ${ }^{14}$}

This study compares functional literacy skills of ALS A\&E Secondary-level learners in BARMM with the national average of OSYA of the same age group. Because there is no data that allows a comparison of skills of ALS learners across the country, the skills level of OSYA in the country generated from FLEMMS is used as a proxy comparator (Figure 17).

The result shows that basic reading and math skills of ALS learners in BARMM are seriously low. For example, about half of ALS Secondary-level learners were only able to solve simple math questions such as multiplying or dividing a two-digit number by a one-digit number while 80 percent of total OSYA of the same age cohorts were able to respond. Also, only 20 percent of ALS Secondary-level learners in BARMM could answer a simple reading comprehension question while over 60 percent of OSYA in the rest of the country responded correctly. BARMM is highly diverse and has many local languages that are 
not necessarily Tagalog or Filipino. The survey respondents reported 14 different mother tongues and this diverse nature of spoken languages may be one key contributing factor to significantly low reading skills.

However, other dimensions measured as part of a functional literacy assessment, such as critical thinking and a sense of belongingness to community/country, were at par with or somewhat higher than OSYA in other regions. Questions related to development of "sense of community" showed slightly higher scores in BARMM than the average of OSYA (Figure 17). These skills are particularly higher among the A\&E program learners, while BLP learners show significantly low skills on these (Figure 18).

Figure 17: Correct Responses (\%) by BARMM ALS Learners in Comparison with Total OSYA, Ages 16-30
Figure 18: Correct Responses (\%) by BARMM ALS Learners by ALS Programs, Ages 16-30

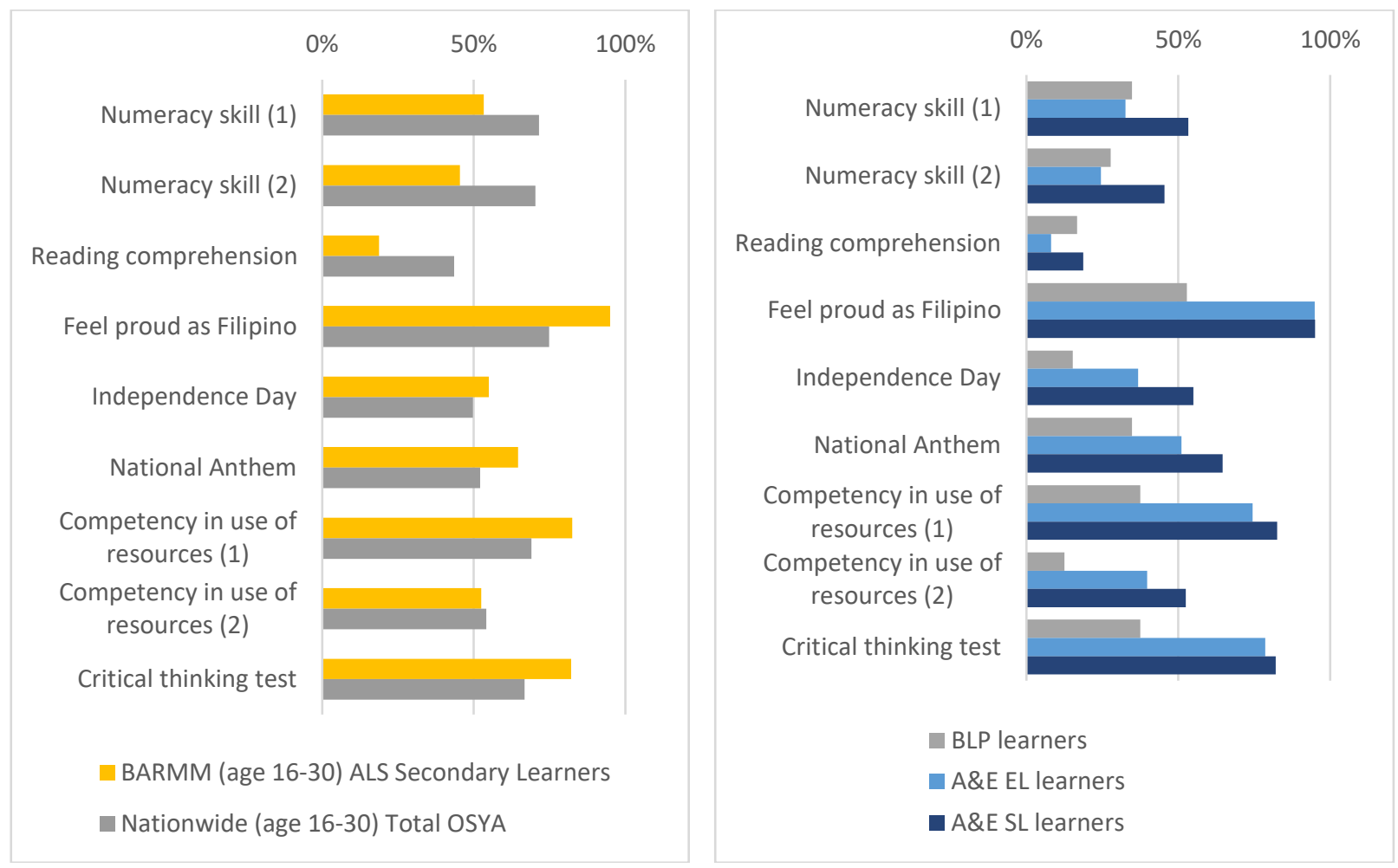

Source: ALS-BARMM Snapshot, 2018, WB; FLEMMS, 2013, PSA.

Note: Only includes those ages 16-30. Same conditions are used to define OSYA.

\section{Socioemotional Skills}

Growing international evidence shows that socioemotional skills (or SES, soft skills) are strongly related to labor market outcomes. Given the changing nature of work, socioemotional skills, besides conventional academic skills, are increasingly focused, and these skills are foundationally critical traits, particularly in the context of ALS. Heckman et. al (2011) discussed that deficiency in non-cognitive (socioemotional) skills limits prospects in the labor market particularly for high school dropouts even if they were able to demonstrate equivalent levels of academic skills on the General Education Development (GED) program.

Thus, the authors assessed their SES by using the same questions derived from the STEP Skills Measurement Survey by the World Bank. The same protocol was enforced and an approach for data editing was followed (Acosta, Igarashi, Olfindo, \& Rutkowski, 2016). STEP gathers self-reported information on personality and behavior. Socioemotional skills in STEP reflect the so-called "big five" 
personality traits: conscientiousness, openness to experience, agreeableness, extraversion, and emotional stability, as well as two additional behavior traits, grit and decision making. These seven traits are expected to influence an individual's ability to achieve goals (conscientiousness, openness to experience, and grit), work with others (agreeableness and extraversion), and manage emotions (emotional stability and decision making). From 2015-2016, the STEP survey was conducted with a nationally-representative sample of working-age (age 16-64) population in the country. The survey included respondents' education level, employment experiences, and demographic characteristics besides direct assessment. In this study, the results are compared to benchmarks as OSYA and the total population ages 16-30.

\begin{abstract}
Box 5: Understanding the "Big Five" Personality Traits
The "big five" personality trait model has been widely used in sociology, psychology, and related fields for over 20 years. During that time, researchers have steadily refined the methodology for measuring openness to experience, conscientiousness, extraversion, agreeableness, and emotional stability. They have also drawn important conclusions about how these skills relate to one another and to the demands of different work environments.

Although each trait has desirable aspects, not all big five traits are appropriate for each professional role. For example, individuals with high scores for conscientiousness tend to be self-disciplined, circumspect, and focused on long-term planning. These are generally positive qualities, but they may not be consistent with success in a position that requires spontaneity or swift reactions to changing circumstances. Similarly, individuals with high scores for agreeableness tend to be considerate and generous, but for that reason they may be reluctant to make hard decisions that involve trade-offs between competing interests. Other traits, such as extraversion, are not inherently positive or negative but merely reflect a specific way of interacting with the world. Some traits, such as emotional stability, are far more valuable in certain contexts than in others. Individuals with high scores for emotional stability may be indispensable in high-pressure situations, but their ability to maintain composure may be less relevant in low-pressure environments. The "big five" construct does not measure the abstract quality of an individual's personality. Rather, it outlines the features of that personality-features that may be more or less valuable, or more or less relevant, depending on the situation (Acosta et al., 2016).
\end{abstract}

The results show that ALS learners in BARMM seem to have consistently lower SES except emotional stability. Research shows that emotional stability tends to be indispensable in high-pressure situations, which may be the case of high scores in emotional stability in BARMM. If compared with total Filipinos with less than a HS diploma of age 16-30, the level of SES BARMM learners is still slightly low and significantly lower than the total population of the same age (Figure 19). There is no significant difference in levels of SES among ALS learners in different programs (Figure 20). 
Figure 19: Average BARMM Scores in Socioemotional Skills Compared to Total OSYA and Population, Age 16-30

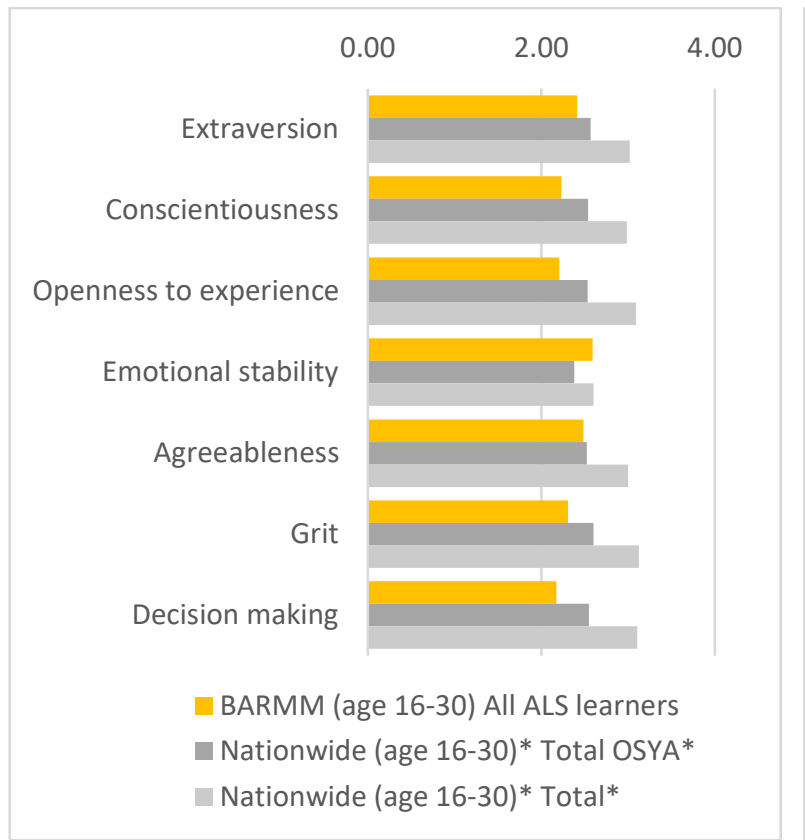

Figure 20: Average BARMM Scores in Socioemotional Skills by ALS Programs, Age 16-30

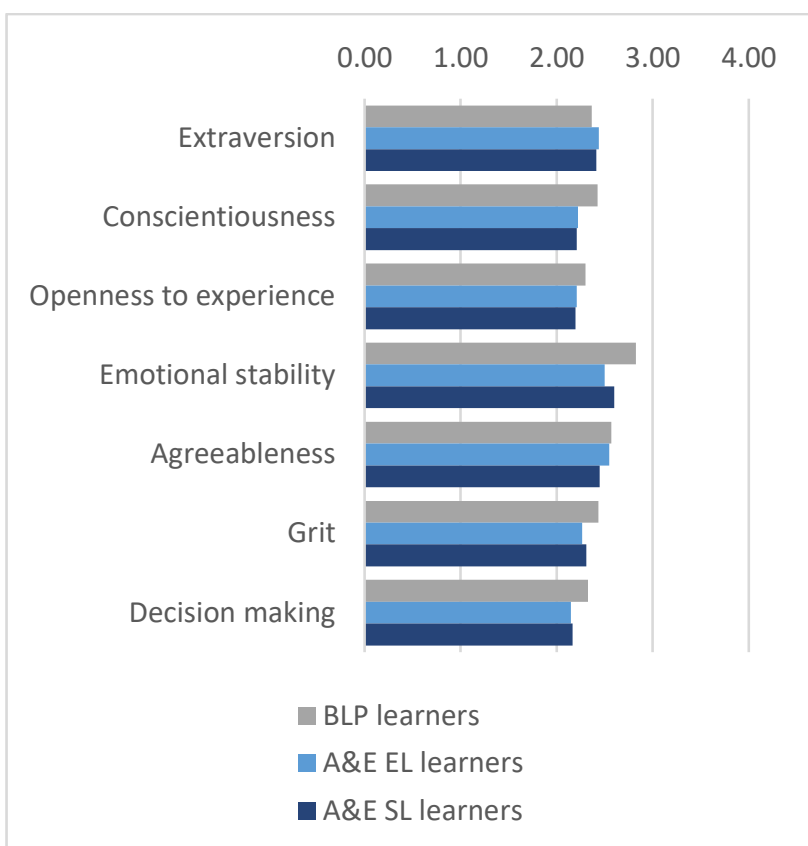

Source: ALS-BARMM Snapshot, 2018, WB; STEP-Philippine Household Survey, 2016, WB.

Note: Includes only ages 16-30. Same conditions are used to define OSYA. Data is on a five-point scale.

ALS learners in BARMM have homogeneous SES distribution regardless of gender, age, educational level or employment status (Figure 21). In the STEP data, the profiles of SES among the labor force were identical between men and women but differ significantly across age groups, educational levels, and employment status. Figure 21 presents a comparison of distribution of conscientiousness across different groups by converting the SES scores to standardized values. Skill distribution is highly similar across various groups in BARMM.

Figure 21: Distribution of Socioemotional Skills of ALS Learners in BARMM by Various Groups

\section{By Gender}

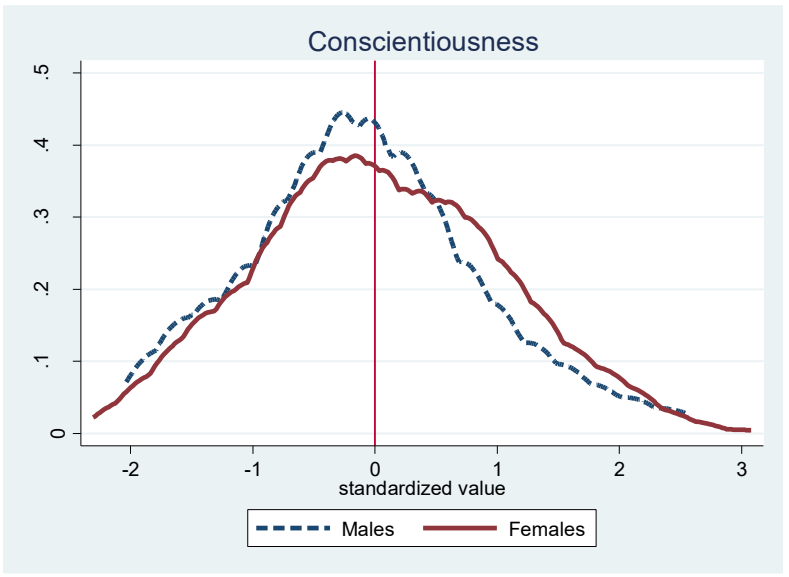

By Age Group

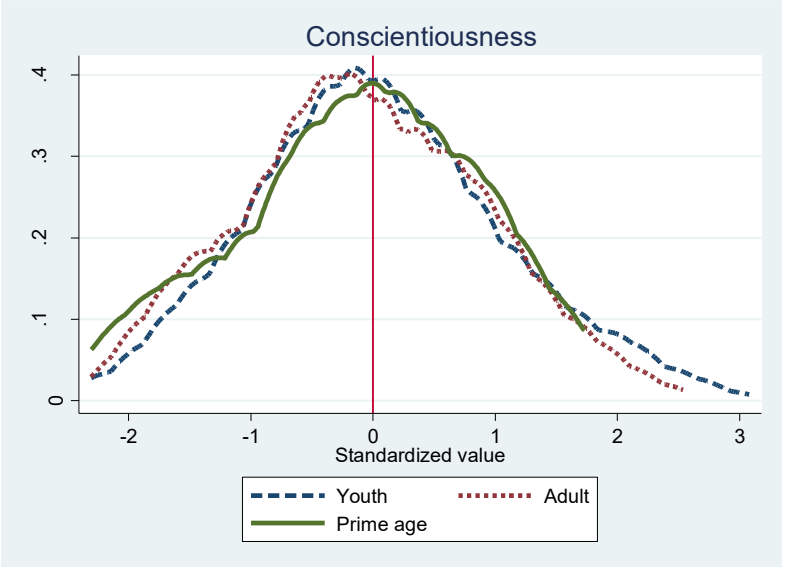


By Education Level

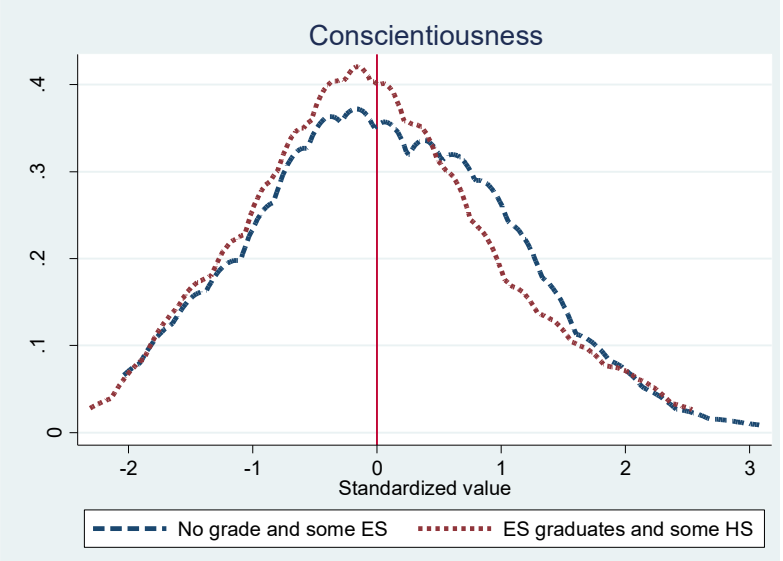

By Gender

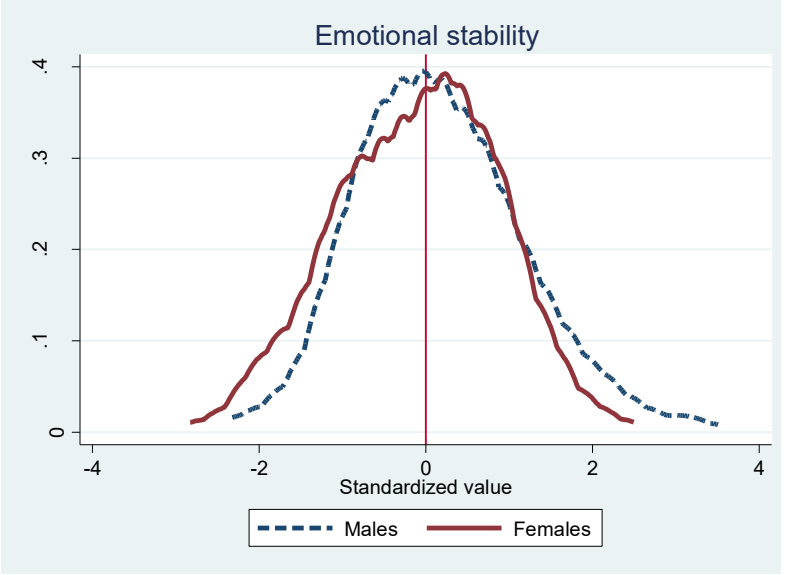

By Employment Status

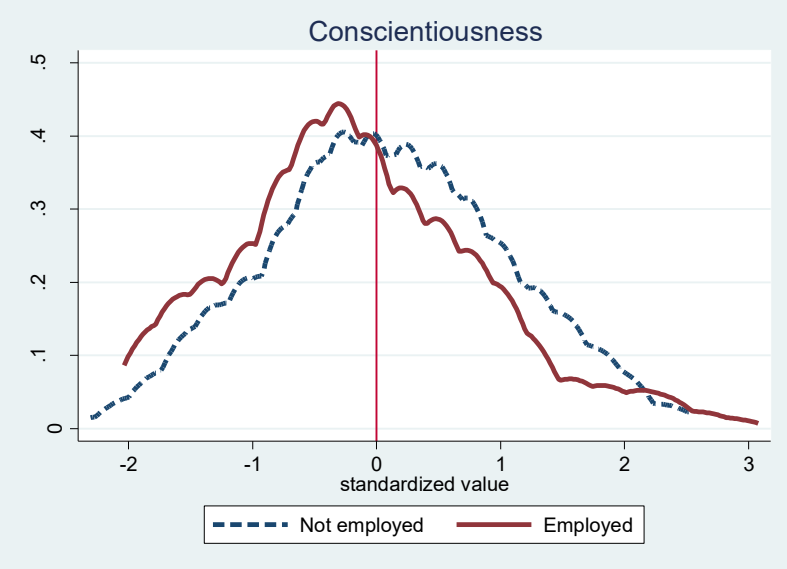

By Age Group

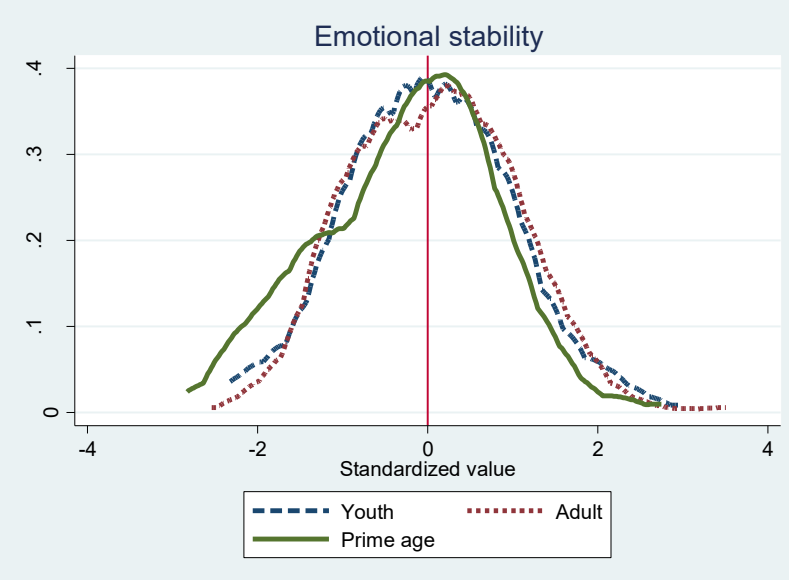

Source: ALS-BARMM Snapshot Survey 2018, World Bank.

\section{Livelihood and Skills Training}

International evidence on adult learning confirms that it is effective to combine livelihood and skills training to adult literacy programs as adults facing opportunity costs see concrete benefits. ALS facilitators have been also encouraged to provide informal skills training to their learners. However, the informal skills training did not receive priority attention by facilitators as well as administrators. Generally, the training was not budgeted unless there were donations from local government units or NGOs.

The data shows that less than half of ALS facilitators provided skills training activities combined with regular ALS learning interventions which led to some form of certification for learners (Figure 22). Most of these training activities are short-term and informal. Forty percent of facilitators reported this additional training led to some form of certification the learners could use for various purposes. The most common focus of these trainings included food processing and beverages, human health and health care, and community development services.

It was also found that these additional skills training programs were funded by facilitators' own funds, which is not optimal as expenses to deliver ALS sessions are also pressing. Only five percent reported that they had some financial sources for skills training from the DepEd. The local government support seems also limited; and other government funds that could be available for skills training such as community-based training by the Technical Education Skills Development Authority (TESDA) was not utilized by any of the ALS facilitators in BARMM. NGO and other partners are active in supporting these 
activities, but their funds are not always available and flexible enough to respond to various demands on the ground (Figure 23).

Figure 22: Share of Facilitators Providing Skills Training for Learners

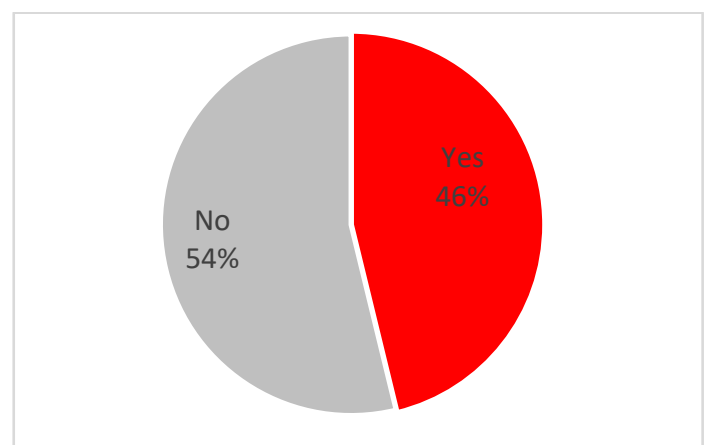

Figure 23: Source of Funds to Finance Skills Training in $A L S$

Source: ALS-BARMM Snapshot Survey 2018, World Bank.

\section{Post-ALS Paths and Aspirations for Learners}

The survey also included questions about learners' aspirations after ALS and readiness to take the next action. This survey was undertaken when they have almost completed the ALS session for 2018, so the information can be helpful for us to think how they can be possibly supported.

Many ALS learners in BARMM have somewhat concrete plans after program completion which differs across ALS programs. Figure 24 presents shares of ALS learners in BARMM by actions to be expected immediately after completing the program. Majority of A\&E secondary level learners have ambitious aspirations to pursue college education ( 52 percent), and 30 percent responded they wish to either return formal high schools, enter technical vocational training programs, or re-enroll in ALS at a higher level. In contrast, A\&E elementary level learners have more diverse plans, but about 60 percent are inclined to more education, primarily entering to formal high schools, TESDA or college. Substantial number of ALS elementary level learners wish to start business after ALS. Finally, we found that close to 20 percent of BLP learners have no concrete plan after ALS, and 30 percent reported that they want to start new business after ALS. Responses, especially aspiration for college, seem far from the reality given the rate of A\&E passers and progression to college among ALS learners. The Philippines passed a law to provide free higher education and TVET in all public institutions since 2018.

Figure 24: Immediate Next Steps After ALS Reported by ALS Learners in BARMM

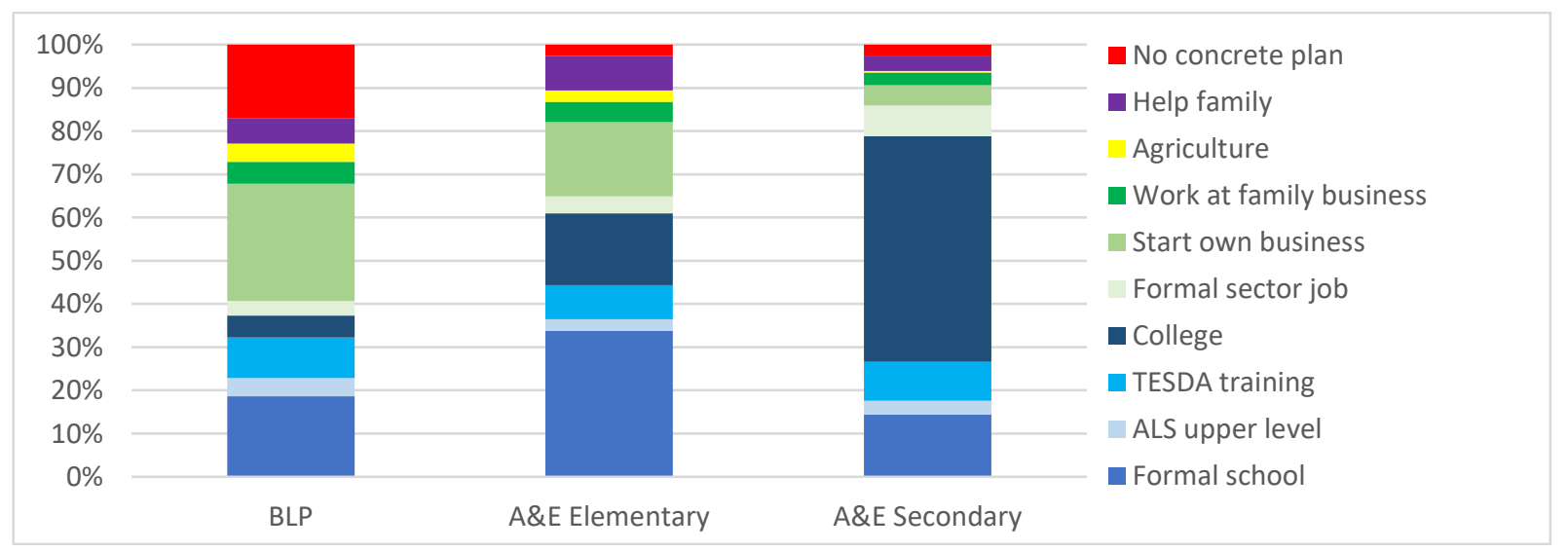

Source: ALS-BARMM Snapshot Survey 2018, World Bank. 
Their long-term career goals are also contrasting by programs (Figure 25). The majority of BLP learners want to start businesses after completing ALS programs. Also, many learners in A\&E elementary and secondary levels opt for regular employment. However, only around 15 percent of A\&E secondary level learners in BARMM reported that they have worked for a job that lasted for three months or more, which is much lower in BLP and A\&E elementary programs.

Figure 25: Long-term Career Goals of ALS Learners in BARMM

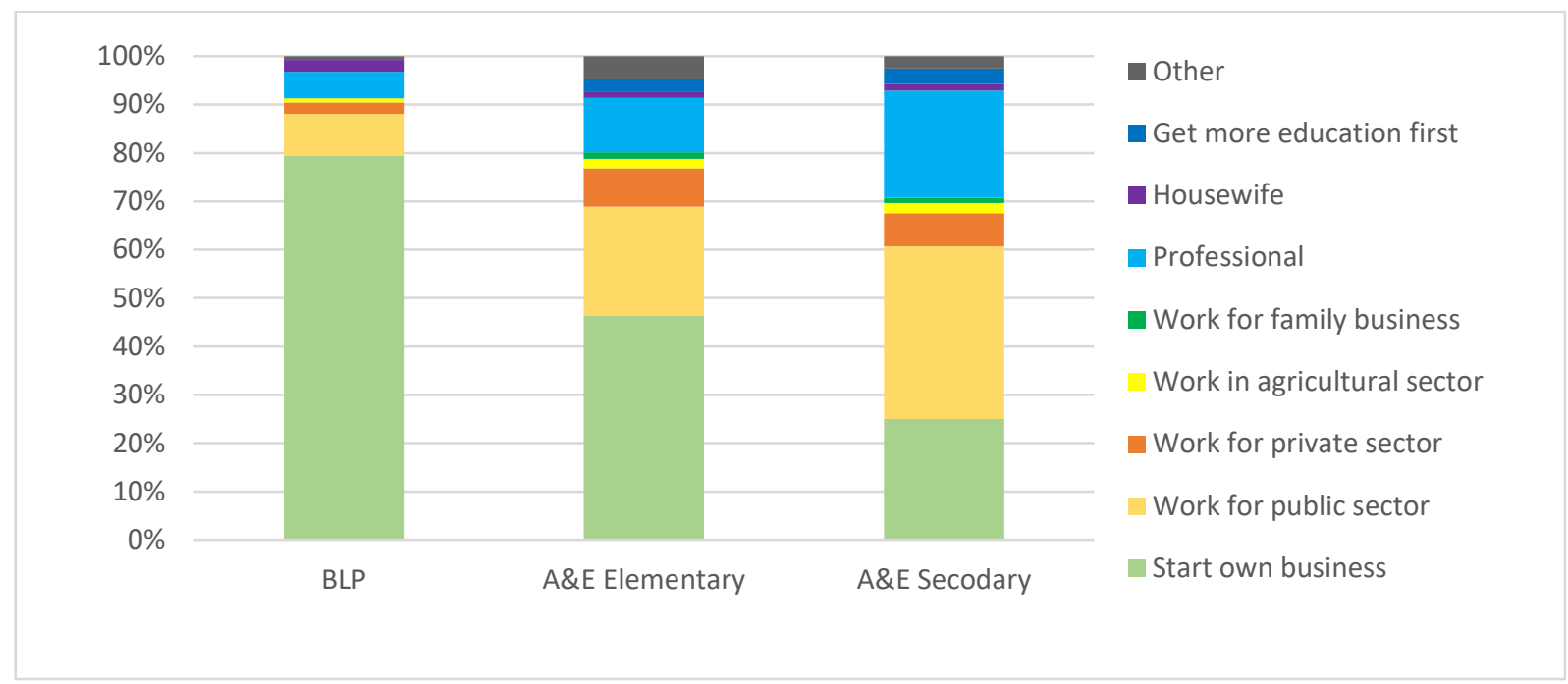

Source: ALS-BARMM Snapshot Survey 2018, World Bank.

While their aspiration is high, the data on the past learners of ALS in BARMM implicates gaps between what is aspired and the reality. The survey also captured whether past learners in ALS in BARMM pursued further education or training after ALS (Figure 26), as well as their employment status a few years after ALS (Figure 27). Only around 15 percent of the overall past ALS learners enrolled in college, vocational or livelihood training or formal high schools. But close to half of those who passed the A\&E tests pursued further education and continue to earn higher educational/vocational credentials after ALS. Also, though small, the employment rates in formal sector jobs are higher for A\&E passers while selfemployed (business owner excluding farmers) is higher for those who are not A\&E passers. In addition, it is important to note that facilitators are actually more aware of the whereabouts of the A\&E passers after ALS, as evident from the data showing the smallest share of respondents reporting "I don't know," which may imply that the passers have clearer personal directions after ALS and consulted well with their facilitators. Though there are clearly advantages among those who were A\&E passers, the reality is that the share of the passers among ALS learners was still less than 10 percent of the overall enrollees in BARMM. 
Figure 26: Share of Past ALS Learners Pursuing Further Education/Training After ALS

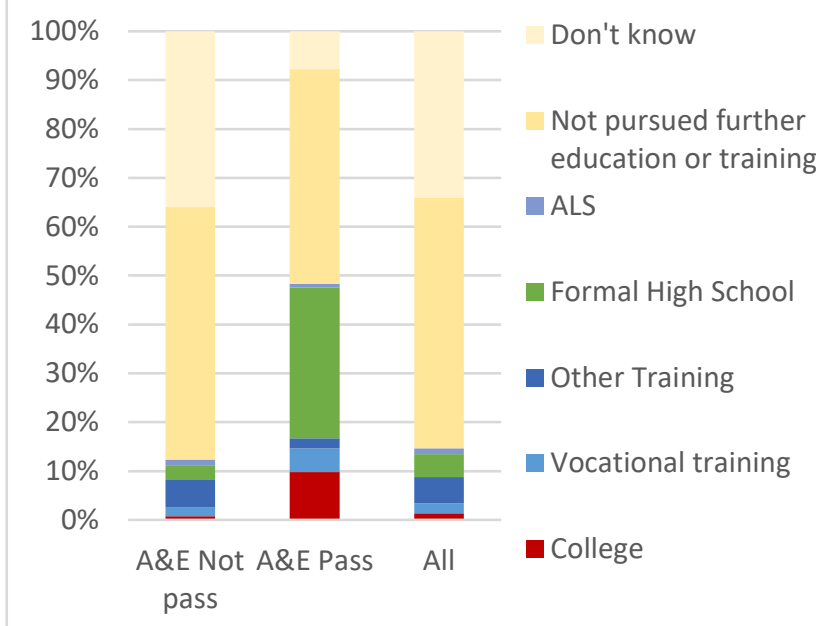

Figure 27: Share of Past ALS Learners' Employment Status After ALS

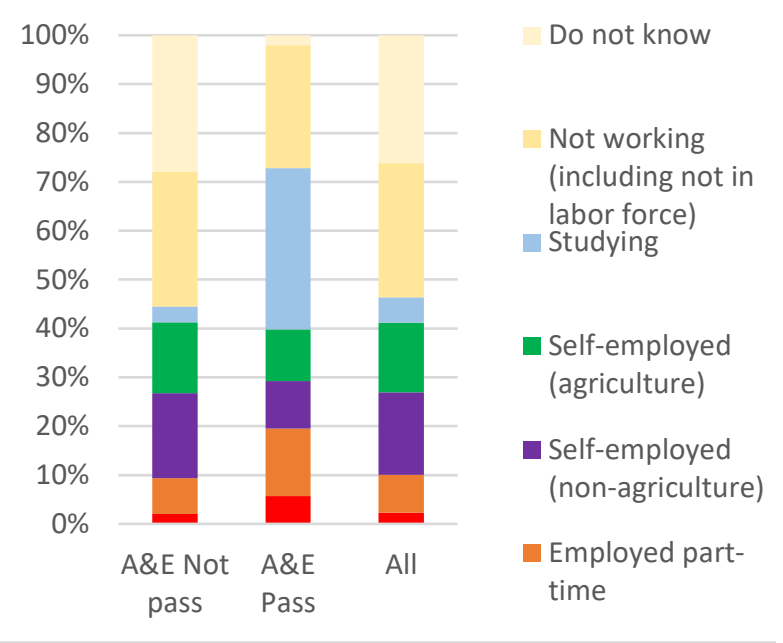

Source: ALS-BARMM Snapshot Survey 2018, World Bank.

About half of ALS learners in BARMM reported consulting with their ALS facilitators on their plans after ALS. Around half of A\&E program learners have had career counseling. About 80 percent of those who had career counseling said that the advice they received was helpful or very helpful. The share of ALS learners who talked about plans or had career counseling seemed low. Aside from ALS facilitators, about half of learners said that they knew of employment services being offered by the national government such as the Department of Labor and Employment (DOLE), specifically Public Employment Service Office (PESO), or the local government units but 20 percent feel that such service is not helpful for them.

Many ALS learners have never had any skills training, and their willingness for more skills training remains very strong. About 80 percent of A\&E Secondary learners and 90 percent of BLP and A\&E Elementary learners in BARMM have never undertaken any formal skills training courses. Over 80 percent said they are willing to take skills training programs. Their preferred a "full-time" program which lasts less than two or three months. The survey also asked their willingness to pay for the program. The average amount (one-time lump sum) they were willing to pay was about PHP600 or US\$12.

Career guidance and information of associated government programs is limited for ALS learners though it could help learners to analyze own interest and aptitudes, make a career choice, and strategize their own practical paths. Outside of ALS, there are public assistance programs, including free public post-secondary TVET and tertiary education programs. As long as one passes the entry requirements, enrolling in public college and public technical-vocational courses is free. However, more than half of ALS learners in BARMM are not aware of these public programs.

\section{Program Delivery}

\section{ALS Program Delivery}

This section reviews the ALS program delivery by analyzing its key system components, which are structured similarly to the formal school system. These "system components" include the overall national budget allocated for ALS, teachers in terms of quantity and quality (or referred as ALS learning facilitators), learning modules like textbooks in the formal system, operational budgets to deliver learning 
interventions, learning facilities and supplies, monitoring and stakeholder participation (Figure 28). In addition, this section highlights the significant governance challenge which has been observed in BARMM.

Figure 28: ALS and its System Components for Effective Program Delivery

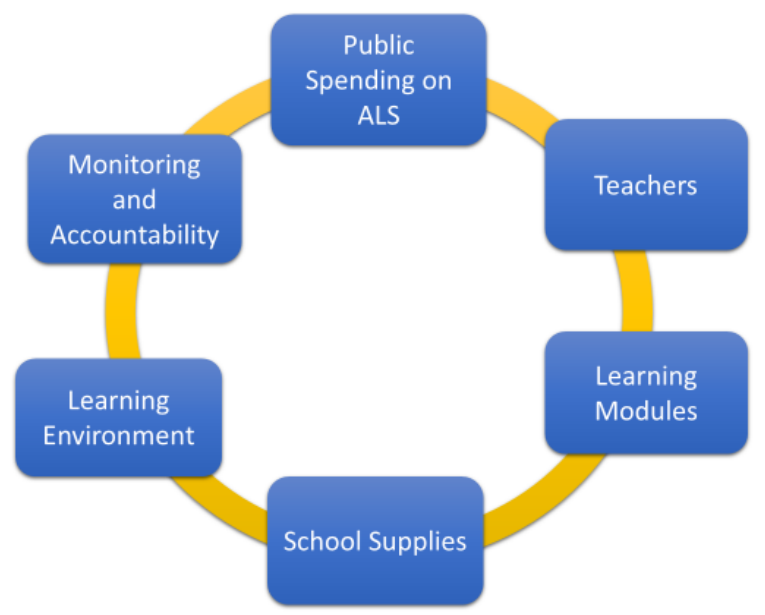

Source: Authors' analysis.

The previous studies found that experiences of teachers, sufficiency of learning modules, quality of learning centers, school supplies, and frequency of on-site monitoring are strongly associated with the outcomes of the ALS programs (Yamauchi, Igarashi, Tenazas, \& Tiongco, 2016; Igarashi, 2017). This seems quite consistent with what ALS leaners in BARMM say about their experiences after undergoing interventions for 10 months in 2018. Figure 29 presents share of learners reporting top two things that they think should be improved in ALS. Across ALS programs in BARMM, basic school supplies (e.g., paper, basic stationary), learning modules (or official ALS self-learning materials), and financial support seem the most significant challenges to learners. Subsequently, content of learning (e.g., relevance to their learning need), learning environment (e.g., building, electricity, furniture), and modalities appear to be problematic, particularly among the A\&E program learners. Demand for context-specific and practical skills training seems highly relevant to ensure learners attracted to the program.

Figure 29: Suggestions for Improvement as Reported by Learners in BARMM by ALS Program

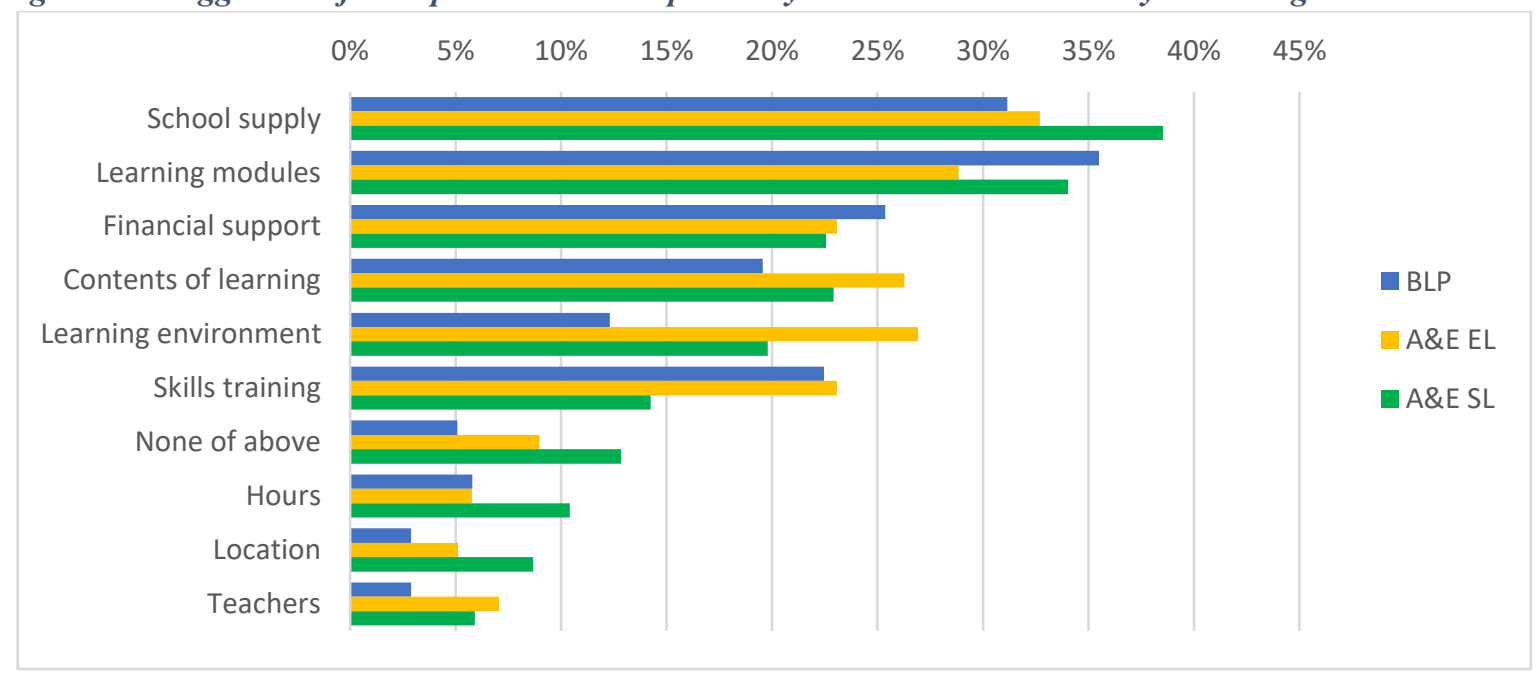

Source: ALS-BARMM Snapshot Survey 2018, World Bank 


\section{Operational Bottlenecks}

\section{Public Spending on ALS}

The national budget for implementation of ALS has been constrained though it has increased tenfold over the last 15 years, but still at a slower pace than the public basic education spending (Figure 30). It marginally increased from 2014 to 2016 but decreased to 0.14 percent in 2017. In 2018, the share of the program's appropriations in the total DepEd budget further decreased to 0.01 percent of the total DepEd budget (Figure 31). With the rapid rise in ALS enrollment and steady increases in national spending on ALS over recent years, the reality is that per-pupil (ALS learner) spending has been lower than the level of a decade ago (Figure 32). ALS has about 0.8 million learners in 2018, which represents three percent of the total students in the country's basic education system, but the allocated budget for ALS remained below one percent of the total public basic education spending for a number of years.

Figure 30: National Spending on ALS in Millions PHP

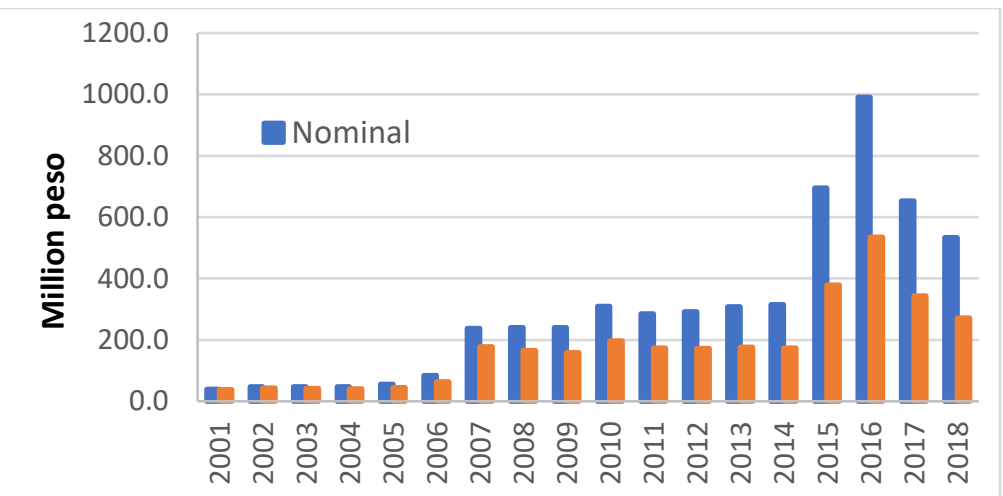

Source: Department of Budget and Management, multiple years; Consumer Price Index, WDI, WB. Note: the real term is adjusted for the effects of inflation to a constant price level using the Philippine CPI.
Figure 31: Percentage of ALS Budget in Total DepEd Budget

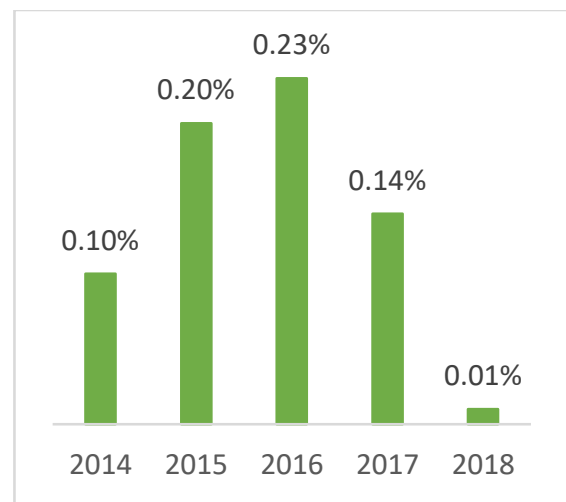

Source: Department of Budget and Management, multiple years

Figure 32: National Per-Pupil Spending on ALS

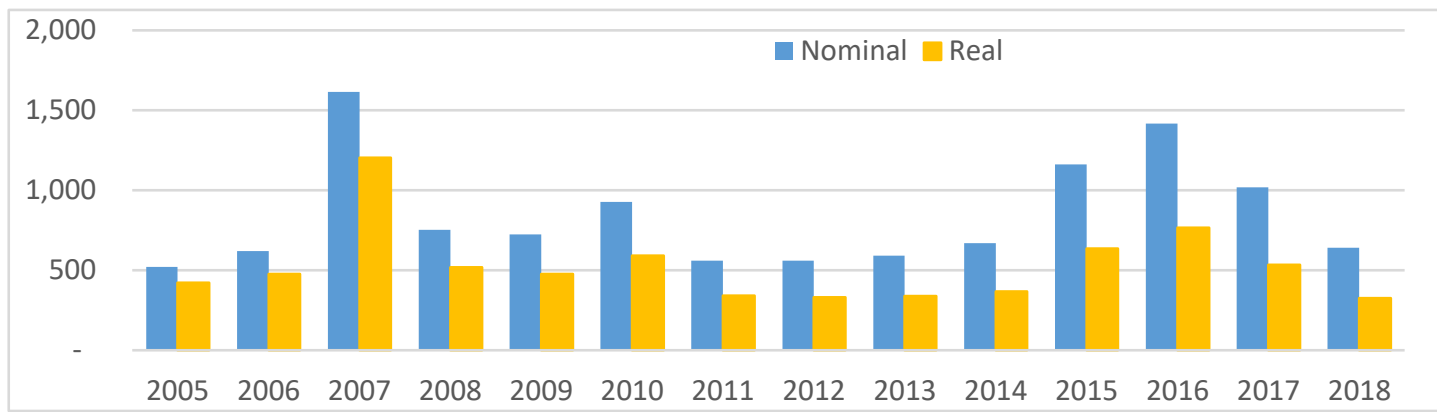

Source: Department of Budget and Management, multiple years; Consumer Price Index (CPI), WDI, WB.

Note: the real term is adjusted for the effects of inflation to a constant price level using the Philippine CPI.

The critical resource constraint in ALS programs in BARMM is owing to low levels of allocated budget to the overall ALS programs. The low level of resources is reflected in the poor quality of inputs for the program (fewer facilitators, less materials; as set out below) and poor outcomes (pass rates, completion rates, labor market outcomes). Upgrading and scaling up of ALS is part of the 10 Point Agenda of the DepEd Administration under Secretary Briones, but inclusion on the policy priority list has not been translated into massive increases in financial investment. Program expansion without substantial resources in place keeps the implementation quality at significant risk. 


\section{Teachers}

As of 2018, BARMM had 283 ALS facilitators, which is the lowest of all regions. Figure 33 show the number of ALS facilitators (those reported to DepEd Central) by regions. Some regions may include teachers funded by sources other than government personal services, but they are not very large in number. ALS facilitators include District ALS Coordinators (DALSC), Mobile Teachers (MT), Instructional Managers (IM), and Literacy Volunteers (LV). DALSC and MT are generally DepEd's permanent employees while IM and LV can be contracted employees or members of education service contractors. In the field, there are also facilitators funded by donors, NGOs, churches, universities, and other public and private entities. In BARMM, there are also donor-funded ALS programs including the ones supported by the Mindanao Trust Fund targeting targeted Moro Islamic Liberation Front (MILF) Camps (Box 6), but the majority of ALS programs are managed and delivered by DepEd's employees. BARMM ALS implementers are comprised of 60 percent DALSCs, 40 percent MTs, and no other positions; thus, clearly BARMM has very limited manpower in delivering the program.

The ratio of ALS teachers (facilitators) to potential ALS learners (OSYA) is the lowest across the county, suggesting a critical challenge in expanding the ALS program in BARMM. As the ALS enroller-facilitator ratio does not show wide distribution across regions, the share of facilitators among OSYA ranged from 260 in CAR to 1800 in BARMM (Figure 34). As per the implementation guideline, each facilitator is required to take up a minimum of 75 learners per year in order to avail operational allowances, and 75 is already a big number for a facilitator to instruct and manage. The available data such as national household survey shows us smaller areas where OSYA are more concentrated, but limited efforts have been made to consider a targeting mechanism to allocate limited teaching positions to areas where OSYAs are concentrated.

Figure 33: Number of ALS Field Implementers by Regions, 2018

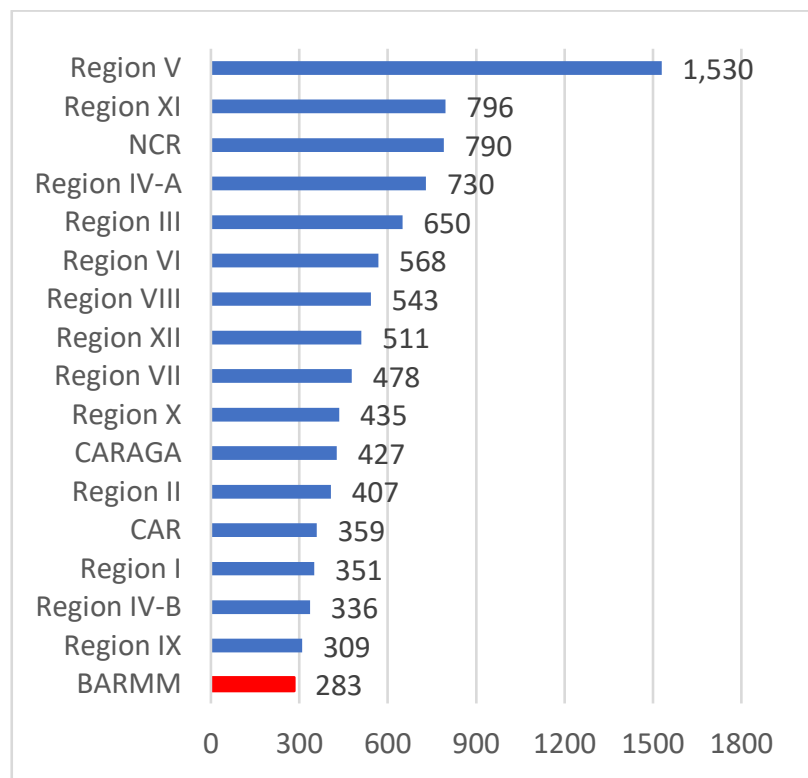

Figure 34: Number of ALS Facilitators as Shares to OSYA and ALS Enrollers by Regio, 2018

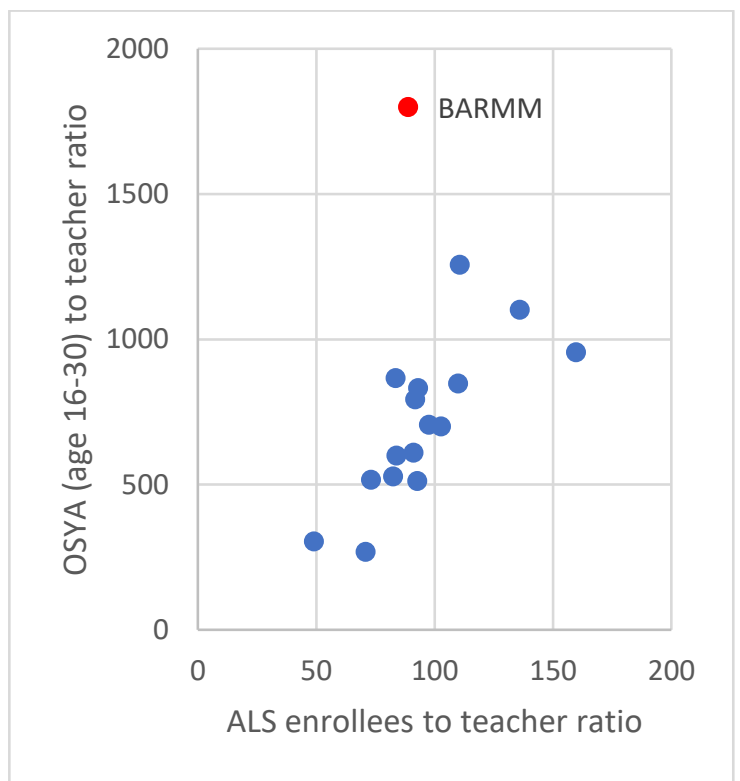

Source: Number of ALS facilitators and ALS enrollers, DepEd, 2018; OSYA estimate, LFS, 2018, WB staff calculation

Aside from insufficient ALS implementers, about 10 percent of ALS facilitators in BARMM are over 60 years old and retiring within five years. This share of elderly facilitators for BARMM is significantly high compared to only three percent in the country. They have longer professional experiences in ALS and developed specific skills, so BARMM will face a significant need to provide induction training for new 
implementers and continuously support their professional development in the field. Moreover, 90 percent of ALS implementers in BARMM are female while the gender ratio is 60 (female) and 40 percent (male) in the rest of the country.

There is a higher share of ALS teachers with advanced degrees but also higher share of teachers with less than bachelor's degrees in BARMM compared to the national average and most facilitators do not have subject-based specialization. As Figure 35 shows, close to 40 percent of ALS facilitators are currently in post-graduate programs or already have post-graduate degrees; however, at the same time, more than 10 percent of MT in BARMM do not have bachelor's degrees. Also, it is important to note that over 70 percent of ALS facilitators do not have subject-based specializations. Particularly English, math, and sciences specializations are scarce among ALS facilitators. This situation may cast potential challenges in delivering the A\&E Secondary Level program as it requires more specialization among facilitators under the new K-12 ALS curriculum. ${ }^{15}$

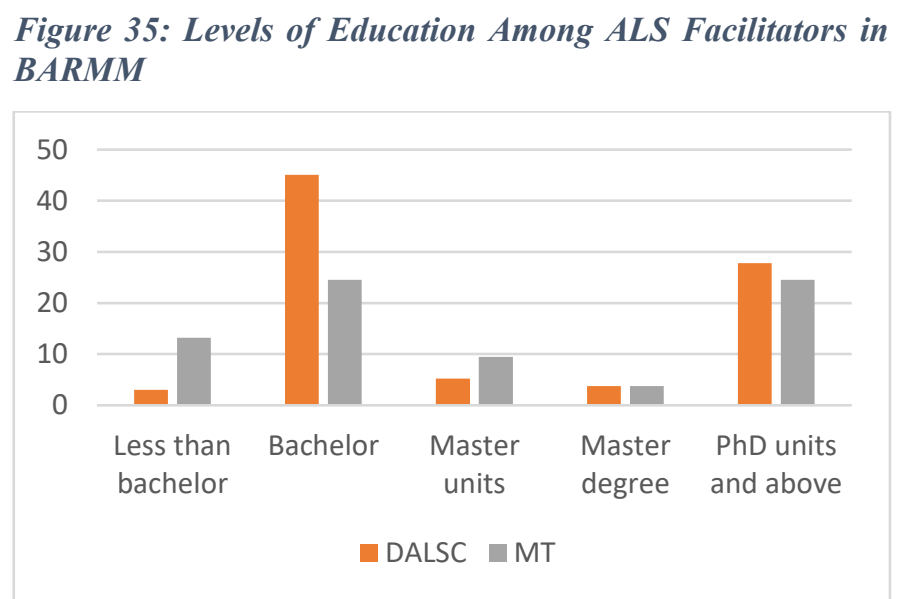

Figure 36: Share of Subject Specialized by ALS Facilitators

Source: ALS-BARMM Snapshot Survey 2018, World Bank.

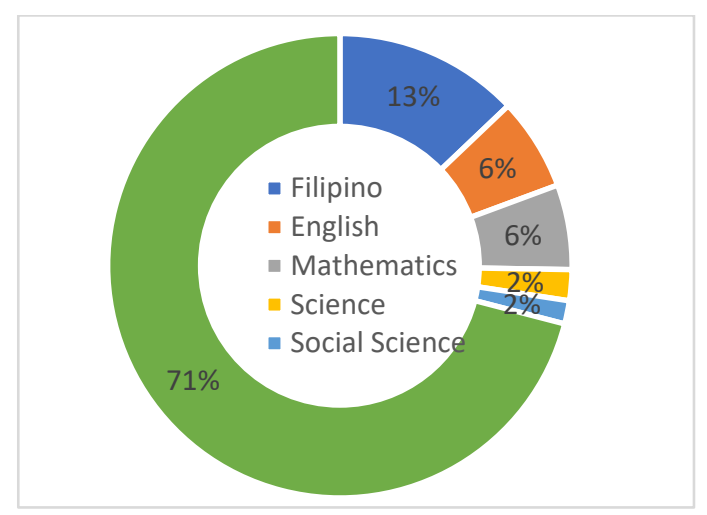

ALS facilitators have limited in-service training opportunities to enhance their skills and often pay to attend capacity-building programs. Figure 37 shows ALS facilitators who attended training programs in 2017 and 2018. The most popular trainings were programs related to ALS, which is the only opportunity for them to acquire new information and meet other fellow facilitators. In 2018, over 60 percent of ALS facilitators reported participating in MYDev Training, a project implemented by USAID on life skills and peace building. Typical modalities of these trainings are mass-training mode which lasts about five days per program. ALS-related training programs were predominantly organized by DepEd BARMM Regional Office, but training programs on other topics were organized by combination of DepEd, public schools, and some NGOs.

The data also shows that facilitators in certain DepEd school divisions have more capacity-building opportunities than other divisions in BARMM. While the sample size does not allow us to precisely describe patterns of training participation at division levels, for example, facilitators in Maguindanao Division I have high participation rates at these major training programs relative to facilitators in other divisions. Also, we found that relatively less experienced ALS facilitators tend to more actively join in these training programs compared to more experienced and elderly facilitators. Facilitators participating in these training programs found these trainings highly useful. Generally, ALS facilitators are working remotely and independently, so this training provides them with important opportunities to not just learn about technical contents, but also to review their own practices and learn from others to overcome some of 
the challenges they face in the field. Opportunities for ALS facilitators to continuously develop their professional capacity may be quite limited and imbalanced in BARMM.

Figure 37: Training Course Attendance in 2017 and 2018

Figure 38: Sources of Funding for In-service Training Courses

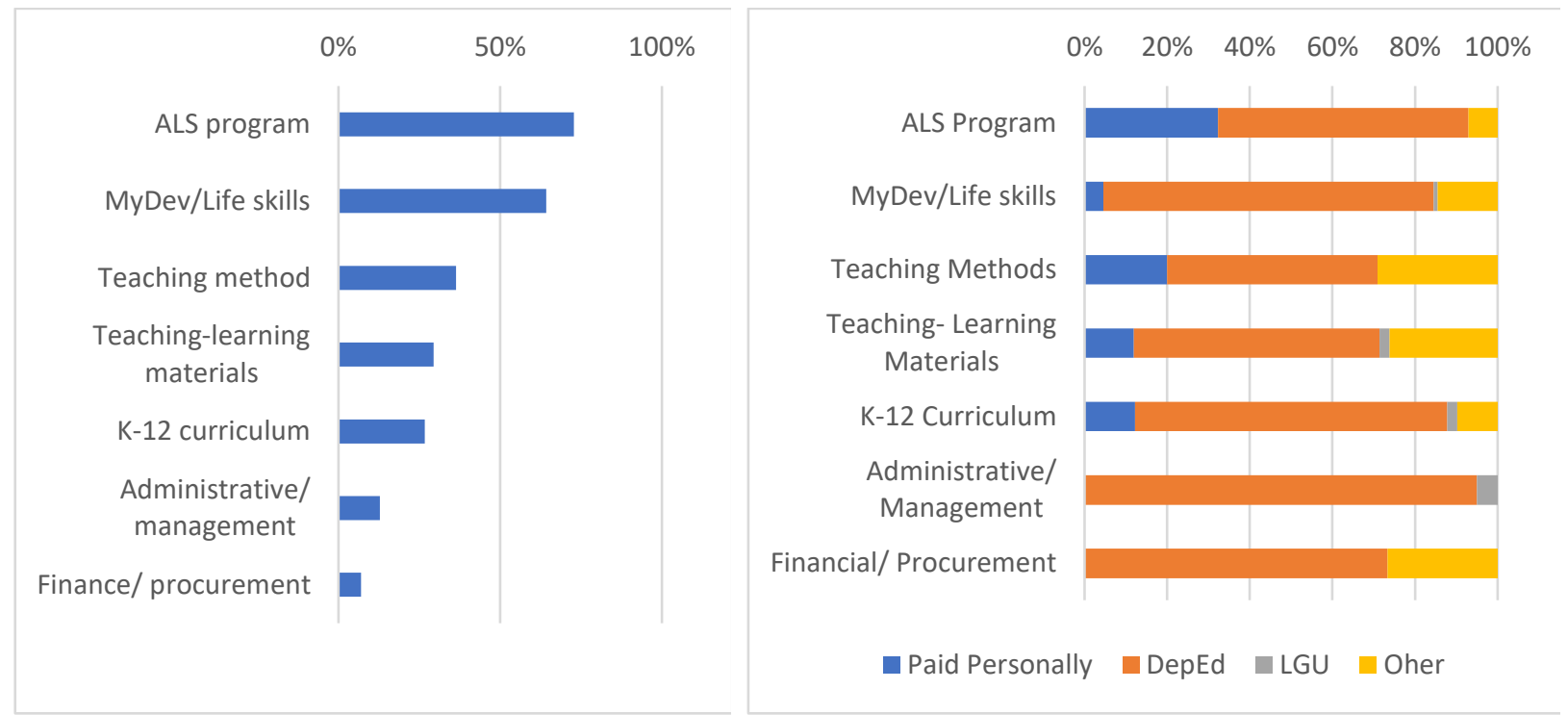

Source: ALS-BARMM Snapshot Survey 2018, World Bank.

\section{Box 6: ALS in Moro Islamic Liberation Front Camps}

Besides ALS programs managed and delivered by DepEd, there are also ALS programs independently delivered by NGOs, donors, and other partners. In BARMM, one example can be the ALS program delivered under the Mindanao Trust Fund (MTF). As a response to the request of the Government of the Philippines, MTF for Reconstruction and Development started in 2003, managed by the World Bank with financial contributions from five countries and the European Union. It has three main objectives: (1) help conflictaffected communities recover economically, (2) help conflict-affected communities recover socially, and (3) strengthen local-level peace-building initiatives.

These activities targeted Moro Islamic Liberation Front (MILF) Camps. MILF's concept of a "camp" is more of a community than a military installation. Activities in the Six Acknowledged Camps included the continuation of community infrastructure and livelihood as well as the provision of the government's Alternative Learning System. Under the MTF-ALS project, about 1,832 former combatants, housewives, and out-of-school youth reported increased confidence because of improved reading, writing, and numeracy abilities. This has provided a model for continued education even under very difficult circumstances. After the Bangsamoro Organic Law was signed in 2018, the immediate concern of education officials was the anticipated influx of rebel returnees and other individuals who will be more confident in joining the rest of society. A strengthened ALS program will be critical in mainstreaming these individuals, as will the improvement of the overall operations of the BARMM Education Ministry.

\section{Learning Modules}

'ALS Learning Modules' consisting of short printed materials are critical as ALS is principally a selfpaced learning program. These materials have been limited on the ground across the country, as well as in BARMM. A set of 283 ALS learning modules, of which 80 are core modules, are provided by the DepEd. 
These modules are supposed to be procured at the Central level and distributed across the country through the DepEd. However, the limited availability of these learning modules is a key bottleneck to ALS implementation in BARMM as well as across the country.

The survey data show that at best only 10 percent of ALS facilitators in BARMM think that they have enough learning modules which is significantly low compared to other regions. As shown in Figure 39, among ALS components, learning modules for the Basic Literacy Program are in especially short supply. More than half of all surveyed facilitators lacked even one complete set of learning modules in 2018 in BARMM. Also, one in every three facilitators own soft copies of learning modules for reproduction or photocopying while they are available on the Learning Resource Management and Development System of the DepEd. Anecdotally, facilitators face significant challenges in reaching the modules they were looking for in the portal for various technical reasons. As reported above, current enrollees also reported shortages of ALS learning modules as one of their top two concerns.

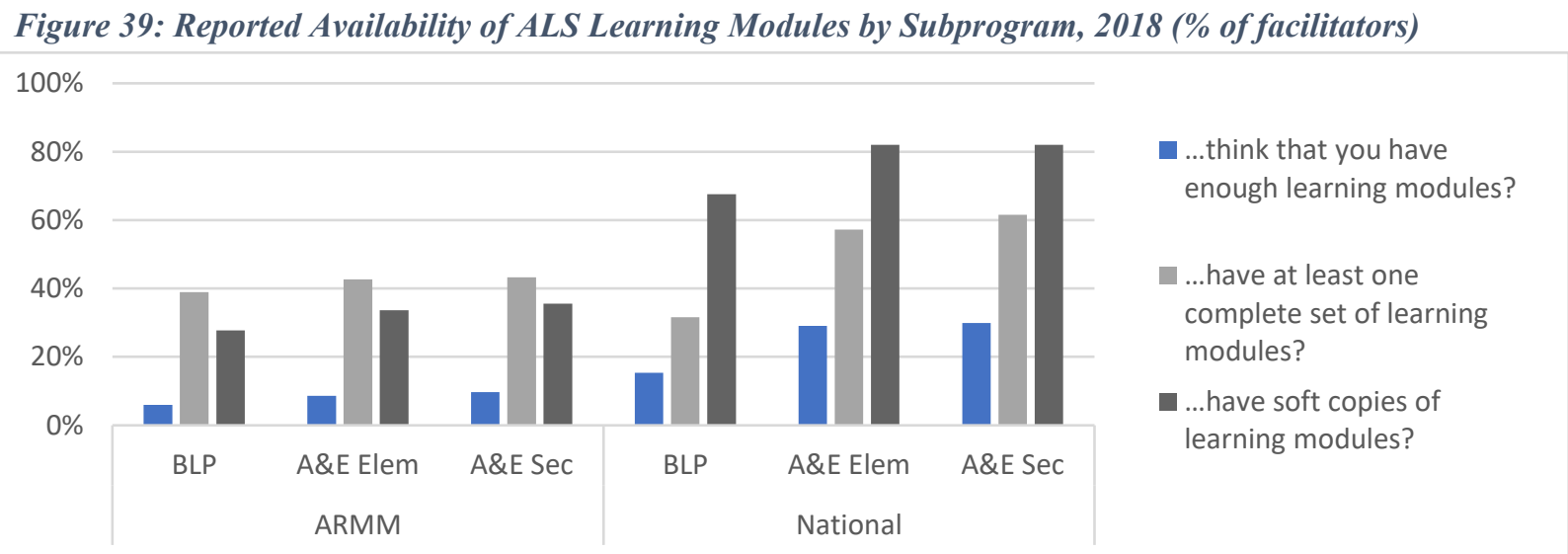

Source: ALS-BARMM Snapshot Survey 2018, World Bank.

Consequently, one-third of BLP learners and only half of A\&E program learners were able to borrow ALS learning modules for self-learning at home to study between ALS sessions. Because adult learners typically require more practice than younger students, the ability to study outside of ALS sessions is especially critical. The inadequate supply of learning modules significantly reduces the effectiveness of ALS programming.

\section{Operational Expenses}

While schools receive the budget to cover operational expenses in the formal system, ALS facilitators receive allowances from DepEd to pay operational expenses and transportation costs to implement ALS programs. Besides these allowances and standard learning modules, DepEd does not provide dedicated facilities, equipment, or supplies, which need to be managed and arranged by facilitators. Many facilitators have cited lack of financial resources as a key constraint on ALS implementation; this section addresses this challenge.

More ALS facilitators in BARMM are paying for a greater variety of expenses to deliver ALS programs using own personal funds compared to their peers in other regions. Over 90 percent of facilitators reported spending funds on basic school supplies, paper, and notebooks for learners (Figure 40). In addition, close to 90 percent of the facilitators reported using a share of their budget to provide meals for learners to encourage their continued attendance. Reflecting serious levels of insufficient learning modules, almost all facilitators reproduce learning modules for their learners. A smaller number of facilitators 
reported covering expenses incurred for livelihood skills training, such as purchasing materials, compensating instructors, and conducting the government assessment for occupation-specific skills regulated by the Technical Education and Skills Development Authority. Facility rental fees and utility costs were the least common expenses.

Figure 40: Facilitator Expenses by Type in BARMM in 2018 (\% of Facilitators Who Reported Spending a Share of Their Budget on Each Item)

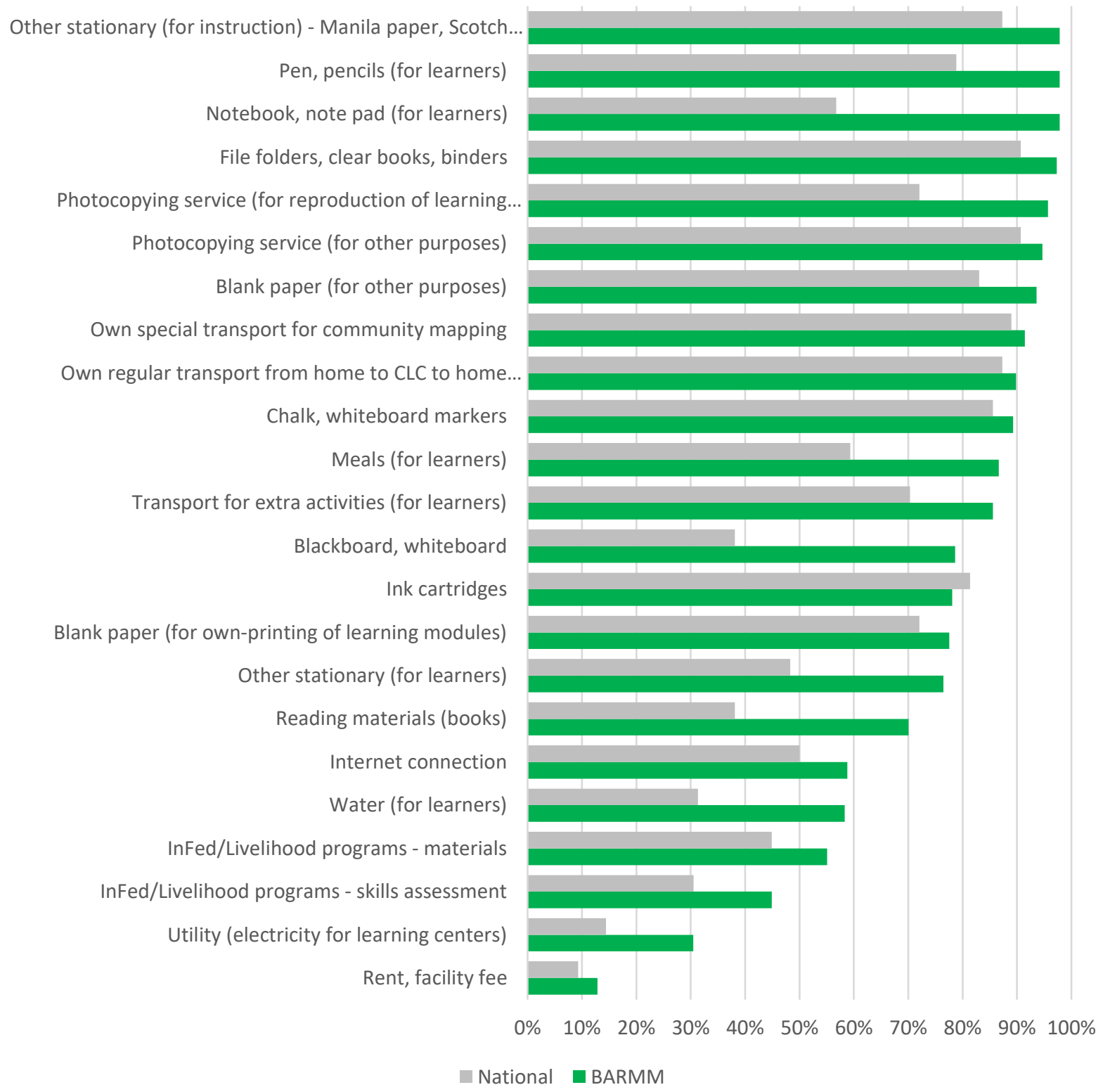

Source: ALS-BARMM Snapshot Survey 2018, World Bank.

Among these categories, transportation costs were by far the largest daily expense as commuting to work place (learning centers) is non-routine and expensive. While top expenses were transportationrelated in other regions, photocopying service fees and meals for learners were significantly high in BARMM (Figure 41). Other significant costs included paper to print instructional materials and learning 
modules for program participants. Although most facilitators reported purchasing stationary and other basic school supplies, these items are relatively inexpensive and represented only a small share of total costs.

Figure 41: Amount Paid Per Transaction on Average in BARMM, 2018 (PHP)

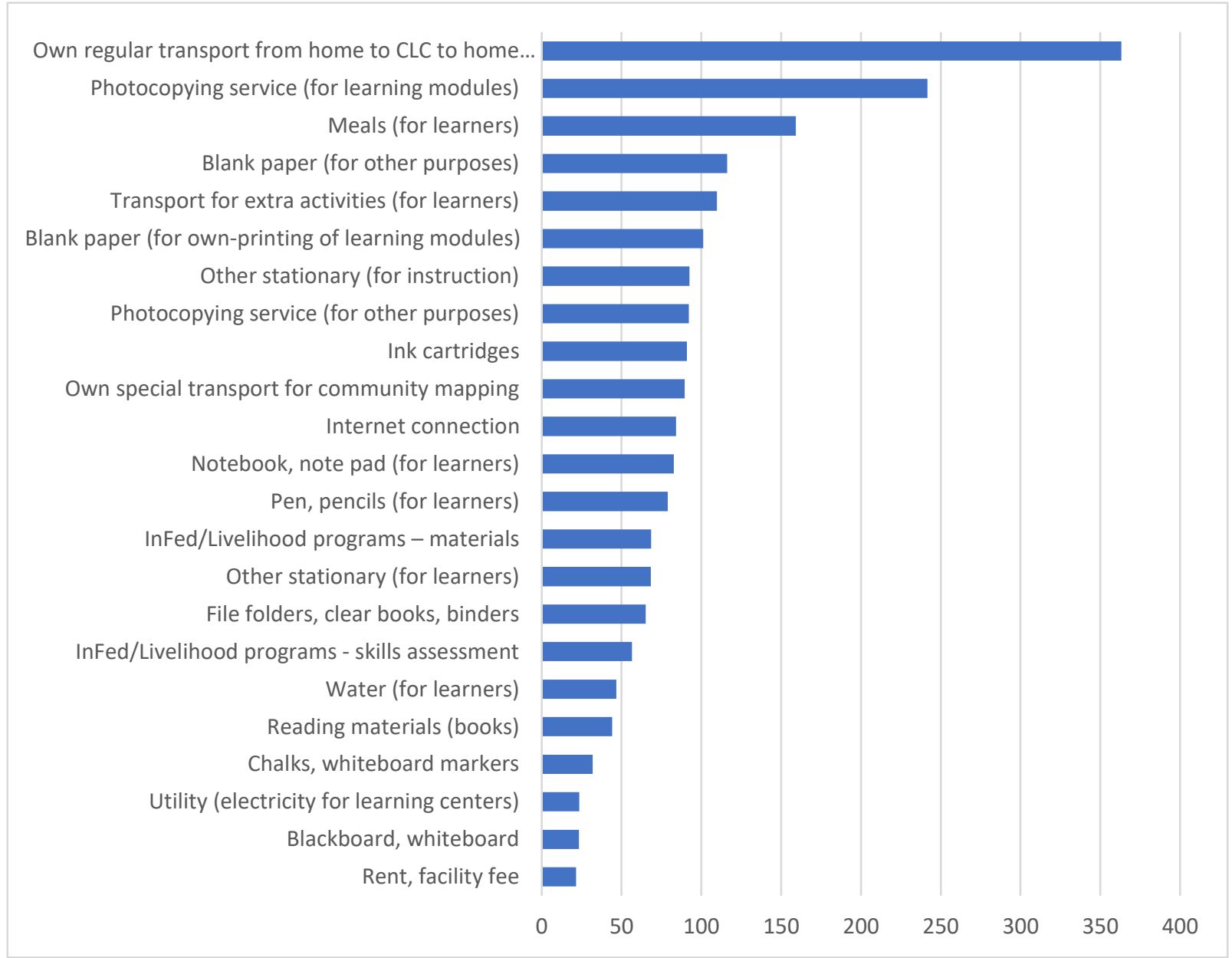

Source: ALS-BARMM Snapshot Survey 2018, World Bank.

The data indicate that facilitators typically spent approximately PHP1,800 per week to deliver ALS sessions, and about 30 percent of that amount went to cover their own transportation costs (Figure 42). This estimate is based on the total amount facilitators spent on the items that over 70 percent of them purchased. The amount they spent for transportation was slightly less in BARMM but does not significantly differ from other regions, but it is important to note that BARMM facilitators reported spending two to three times more on reproducing learning modules than did facilitators in other regions. As noted above, the estimates of total operational spending exclude items purchased by less than 30 percent of facilitators.

These expenditure estimates significantly exceed the amount of funds allocated to ALS facilitators by DepEd. The financing gap averages about PHP1,000 per week, combining both transportation and nontransportation spending. Gaps between the amount provided for teaching aid and actual non-transportation expenses were larger than the gap between transportation allowance and actual transportation expenses. As mentioned above, almost all facilitators in BARMM spent a large amount of their budget on reproduction of learning materials. If that part is removed, the gap is somewhere around PHP500 per week. On an annual basis, expenditures would exceed funding by as much as PHP20,000 (US\$400) per facilitator even if 
learning modules were fully provided. Most facilitator expenditures are devoted to covering basic operational costs, leaving few resources to purchase supplemental learning materials. Beyond the DepEd training modules and essential school supplies, the use of additional textbooks, exercise books, and computers could greatly enhance educational outcomes. However, few facilitators have the resources to purchase supplementary materials. Instead, most ALS funding is spent on essential educational materials and transportation costs.

Figure 42: Total Weekly Spending by ALS Facilitators in BARMM, 2018 (PHP)

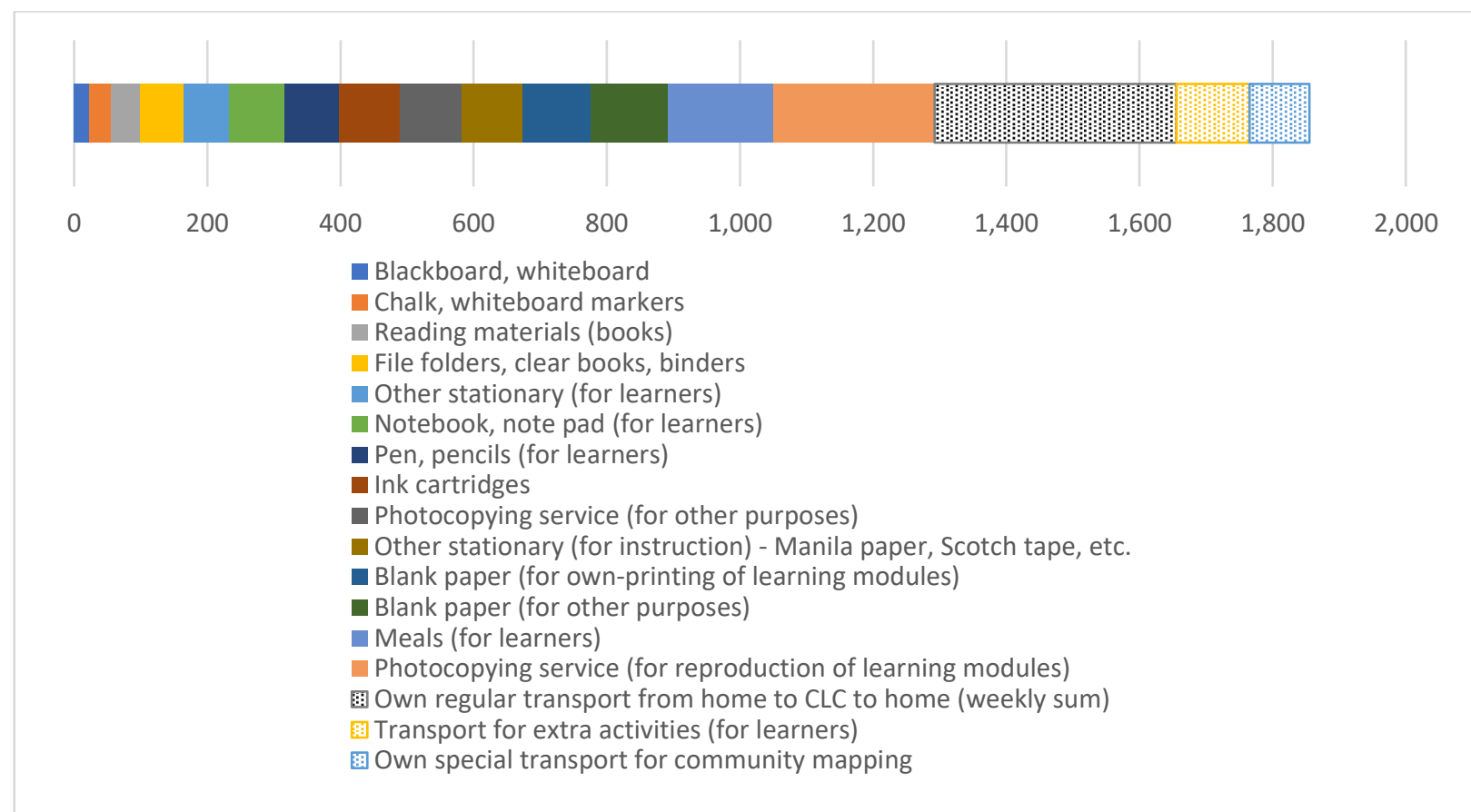

Source: ALS-BARMM Snapshot Survey 2018, World Bank.

\section{Learning Environments}

Because the ALS generally does not use dedicated public facilities, ALS facilitators convene classes in a range of venues. The learning environment for ALS enrollees varies substantially. ALS programs are held in public schools, community centers, and other public buildings, as well as private homes, churches, sports fields and other outdoor spaces, and other temporary and permanent locations. Of these facilities, only public schools are owned by the DepEd. All other venues are provided by local governments, community organizations, or private individuals. The DepEd classifies ALS learning environments into five types, ranging from the most basic to the most sophisticated (Figure 43). 
Figure 43: Distribution of ALS Learning Facility by Type

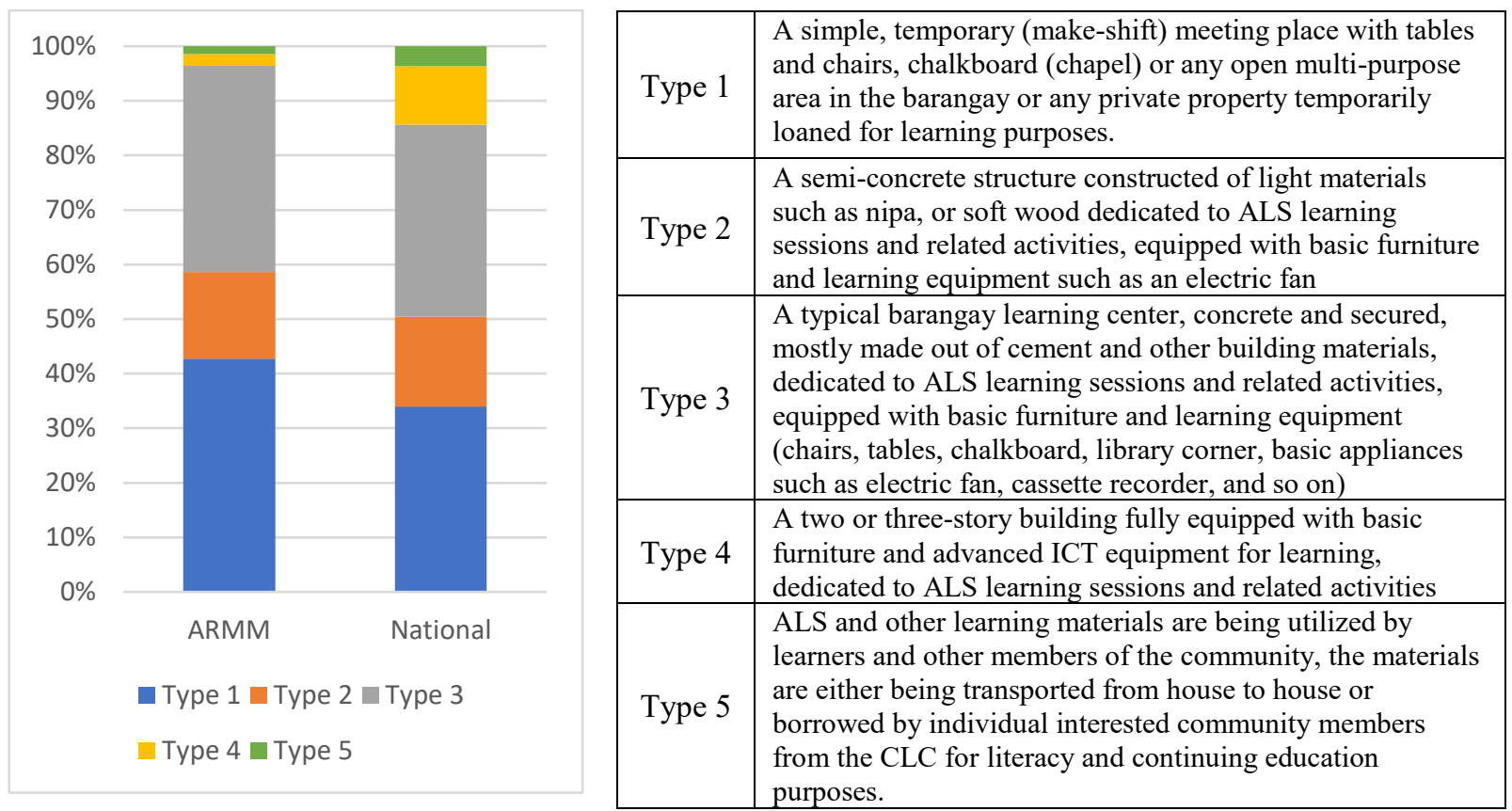

Source: ALS-BARMM Snapshot Survey 2018 (ARMM); World Bank; ALS Snapshot Survey, 2017 (National); World Bank.

Conditions of ALS facilities are worse in BARMM compared to the country as a whole. In BARMM, the most common learning centers (about 60 percent) are basic structures (or no structure) and temporary locations (Type 1 and Type 2), while structures that are more permanent, more secure, dedicated to ALS, and has information and communication technology is 3.5 percent in BARMM against a national average of 15 percent (Figure 43). Type 1 and Type 2 are the most likely to be rendered unavailable by extreme weather or other adverse conditions. These also do not have any security features, making it difficult to store materials and other equipment.

In theory, ALS programs could be held in any facility type, but in practice differences in the venue tend to affect both attendance and program quality. A simple regression analysis revealed key supplyside challenges to improving the quality of ALS programming (Igarashi, 2017). The analysis controlled for facilitators' gender, age, years of ALS experience, and administrative division. The outcome variable was the attendance rate per facilitator. The analysis showed that spending on teaching supplies and the type of facility both affected the attendance rate. The best equipped facilities (Type 5) were associated with a 19 percentage-point increase in the likelihood that participants would complete the program. The results clearly indicate that better learning environments and increased funding for educational materials have a significant impact on program attendance and completion. This is consistent with the international literature, which found that noise, distractions, or uncomfortable environments cause deficits in cognitive performance among adult learners. While it should be noted that the analysis in this note did not definitively establish a causal relationship, it is reasonable to infer that the quality of learning facilities and educational materials influence participants' ability to make the sacrifices necessary to complete the program.

\section{Monitoring and Supervision in ALS}

Research shows that regular monitoring of learning facilitators is essential to ensure program quality in ALS and defining how and for what monitoring is undertaken is important. There is a significant and positive association between the frequency that facilitators are monitored and their time commitment, 
which is closely related to greater gains on the indicators in ALS (Yamauchi et al., 2016). However, the diverse environments in which ALS facilitators operate - which include isolated indigenous communities, remote mountain regions, and conflict-affected areas - greatly complicate oversight. ALS supervisors have difficulty monitoring programs in communities that are remote or difficult to access and are located remotely principally.

However, the data shows that monitoring seems to not be optimally coordinated with 14 percent of ALS facilitators in BARMM reporting they were never supervised during the last two years. The data shows that public schools (such as schools heads) and district supervisors pay the most frequent visits to ALS programs as reported by facilitators in BARMM (Figure 44). On average, facilitators were monitored by school principals or district supervisors over 10 times per year on average. There is a statistically significant and positive relationship on the frequency of supervisions. This means that facilitators who are monitored by school are more likely to be monitored and visited by other monitoring agents, leaving other facilities with limited chances to be monitored and given feedback for improvement.

Figure 44: Number of Monitoring Visits to ALS Activities by Agent per Year

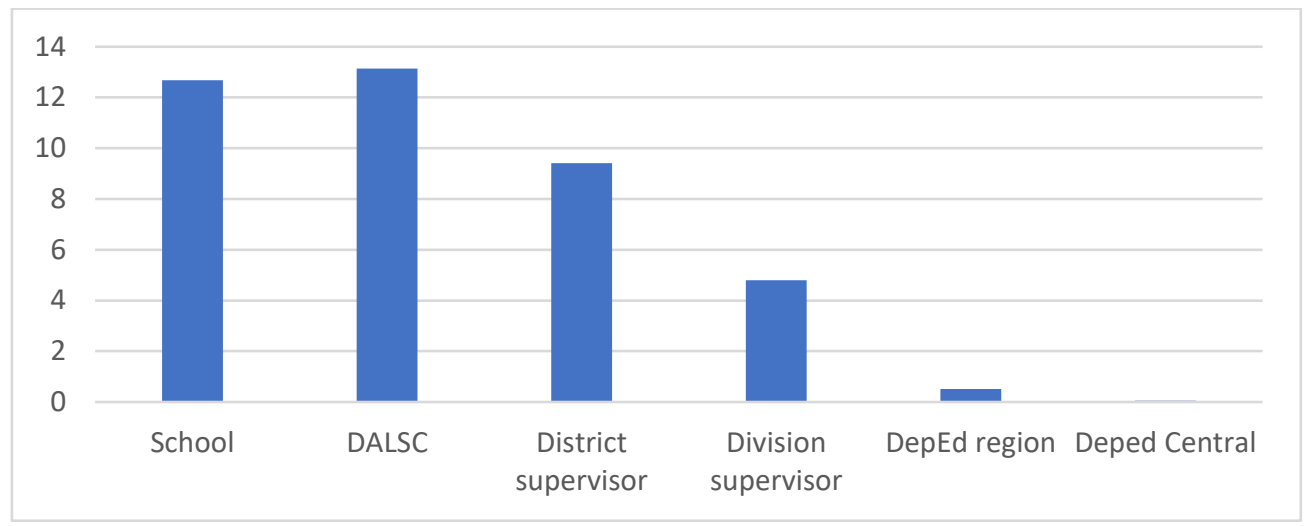

Source: ALS-BARMM Snapshot Survey 2018.

While the majority of facilitators are moderately satisfied with feedback received during such visits, about 15 percent found it not so useful. On-site monitoring is costly and difficult for ALS, but the reality seems that there have not been systematic practices which define how to undertake monitoring and what aspects should be monitored on the ground. Facilitators seem to be missing important opportunities to be objectively observed and given important feedback for their development. Moreover, about one out of four ALS facilitators in BARMM reported that they did not have their performance review for 2017. It is a significant number, but the survey lacks the data to explain why the reviews were not conducted. The average rating for the feedback received from the performance review shows that 10 percent of facilitators gave the lowest scores (for instance, the visits were not helpful at all).

\section{Special and Irregular Challenges}

The survey asked a set of questions to understand the concerns related to irregular or illegal practices in BARMM, which have been shared from the field. The education sector comprises a large share of BARMM's budget, and this typically makes the sector prone to corruption. These issues in the formal education sector have been also reported in various reports including the one by the WB (2015), but none has looked at the situation in ALS in BARMM. The questions in the survey were adapted from the World Bank's public expenditure tracking survey, standard exercise to diagnose leakage of public funds and contextualized carefully in consultation with field implementers (WB 2016). 
The results show that about one out of five ALS facilitators agree that making a gift or donation is common practice in order to have an official appointment. In addition, the same share of facilitators reported that giving 'improper' incentives is prevalent in expediting the paperwork process that is necessary for them to gain their benefits such as retirement, promotion, and scholarships (Figure 45).

Besides, these behaviors seem systemic particularly when facilitators receive operational allowances that are critical to deliver learning interventions. About 10 percent reported that they usually have to give such incentives to receive their operational allowances, and close to 20 percent have been actually asked to donate some in order to receive their allowances for the reasons including contributions for renovating offices. The operational allowances (e.g. Teaching Aide and Transportation Allowances) provided directly to facilitators are smaller than the actual need as we saw, and this may affect the availability of necessary supplies for learners. We also found that on average PHP900-1,100 was paid to receive their allotted allowances.

Furthermore, at the time of the survey (November 2018), there was no facilitators who confirmed the receipt of the allowance for 2018. Part of the reasons why these payments have been tolerated reflect the fact that these operational allowances do not reach facilitators at the beginning of interventions but arrive towards or after completion of the programs. Another reason for this practice is that other DepEd officials do not individually receive such transportation and teaching allowances. According to the DepEd Central, the fund was downloaded to the regional level long before that time, so serious bottlenecks lie in disbursing the necessary funds from the regional level to the divisions and individual facilitators.

Figure 45: Irregular Practice as Reported Figure 46: Irregular Practice as Reported by Facilitators II by Facilitators
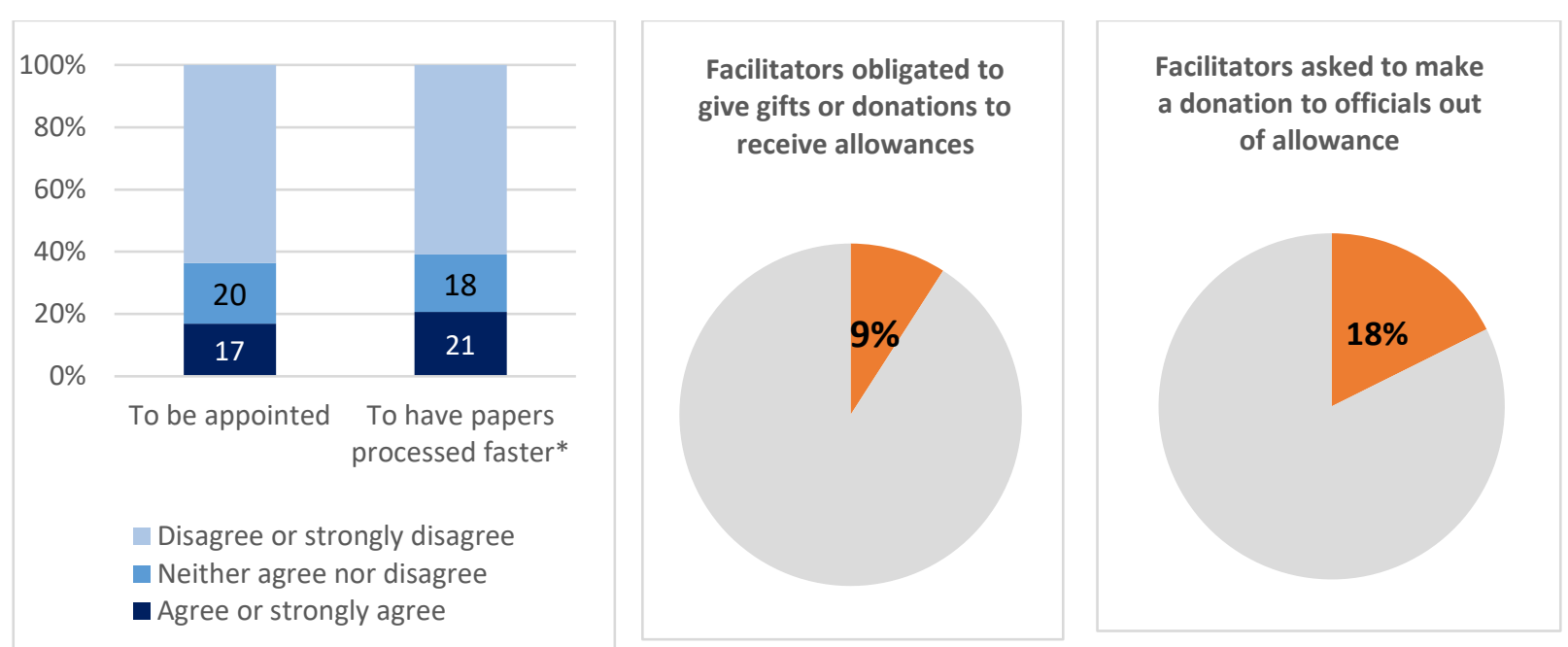

Source: ALS-BARMM Snapshot Survey 2018, World Bank.

Although the study was not a formal investigation, the issue of "ghost" teachers was also seen in a few small areas in BARMM during the survey. The survey team used the master list of ALS teachers and randomly selected responds for this study. In some areas, the team encountered situations where some ALS teachers could not be traced or located though were listed in the official record. They are apparently inactive in reality or unknown to other fellow teachers working in the same areas. Similarly, there are also other teachers whose status were never after several attempts to follow up by the survey team. Based on the survey, 8 percent of ALS teachers in selected divisions in BARMM are of questionable status.

Also, there are several instances that their learners were not actual learners, or ghost learners. They are registered in the system but never or not actively attending learning sessions. The study team discovered 
the existence of these learners by enforcing the survey protocol strictly. Existence of these learners allow teachers to receive their operational allowances even if they have not served the required number of learners.

These ghost teachers' and learners' issues were only found in two provinces rather than scattered across BARMM. Needless to say, their presence represents a significant violation of government service delivery and in the context of very limited resources for the ALS program, and the massive demand for ALS in BARMM, this is a very significant leakage in the system. Box 7 provides more insights and recommendations to these issues.

\section{Box 7: "Ghosts" in ALS: Circumventing ALS service Delivery Standards in BARMM}

The crux of the problem with ghost individuals is information asymmetry. BARMM suffers from four distinct phenomena: first, it has unreliable public utilities like electricity and information and communication technology (ICT), which make information transmission significantly slower compared to the rest of the country. Second, it has less-developed road networks (except in special cases like the Basilan circumferential road) which makes travel to far-flung areas very difficult. Third, it is perceived to be dangerous for outsiders (and sometimes even for locals), so even if road or boat networks are available, the interest to travel is low. Lastly, it was also perceived to be desensitized to corruption, making the integrity of some information coming from the field suspect. The presence of all of these factors makes an environment conducive for ghost individuals.

The following are recommended for addressing the specific problem of ghost teachers and fake learners:

- Improve information dissemination: The economic fundamentals of the BARMM, and a major part of this is having reliable utilities in the entire region. Significant improvements in ICT infrastructure will enable to manage information across the BARMM, to align incentives better and minimize misconceptions in the intentions of various activities.

- Strengthen M\&E: Monitoring and evaluation functions should be urgently strengthened. ALS teachers are seldom visited, and current monitoring systems are outdated. This M\&E should include annual program audits for verification, and unannounced on-site monitoring on a random basis could be explored. Particularly in the areas where they see persistent and prevalent corruption issues, engagement with civil society groups and local community in monitoring activities should be critical.

- Prosecute erring officials: As part of stronger M\&E, existing laws should be enforced. Corrupt individuals and all their accomplices should be brought to court and given the appropriate punishment if found guilty. It is common knowledge that these illegal practices are propagated because people at both higher and lower levels from the corrupt individual benefit from the scheme. Prosecuting all those involved in wrongdoing will also strengthen the perception of a stronger rule of law in the new region and help deter future offenders.

- Improve education policies: Some policies produce unintended negative consequences. In terms of ALS, the first among these policies is the 75-learner requirement for all implementers. A formal school classroom is targeted to have only 40 students of the same grade in the same place, and teachers are only expected to teach for 6 hours a day. On the other hand, mobile teachers are tasked to teach 75 individuals with varying learning needs, across as many barangays as necessary, during learning sessions of 3 to 6 hours per day. Unless this is changed by better policies and commensurate investments in ALS, there will always be an incentive to produce ghost learners. 


\section{Policy Recommendations and Conclusion}

The new BARMM government will face a number of major challenges in the education sector. The large number of out-of-school youth, both of school and over-school age, is testament to a legacy of poor quality and inadequate access to basic education. These deficiencies in the formal system require urgent action. Many children and youth in BARMM will continue to need alternative learning modalities as a complementary pathway for continuing their schooling and completing their missed learning. Thus, harmonized efforts for the formal school education, ALS, ADM are expected to alleviate both the flow of school dropouts and the stock of OSYA.

\section{Recommendations}

Significant changes need to be made to improve the quality and effectiveness of the ALS system as it operates in BARMM. Making these changes should be given the highest priority, after which efforts to expand the program can be undertaken. Given the huge potential demand for ALS, tackling the quality issues is urgent.

Continued strong collaboration between DepEd and the BARMM Ministry of Education on ALS will remain foremost important. The national level has a significant role to play not just in allocating resources, but also in setting the strategic direction and policy, as well as refining system components in pursuit of ALS Version 2.0. On the other hand, the BARMM government should continue to collaborate in implementing key policies and programs while contextualizing them as appropriate to address the unique concerns in the region. The recommendations from this report are summarized in the simple diagram below and Table 6 .
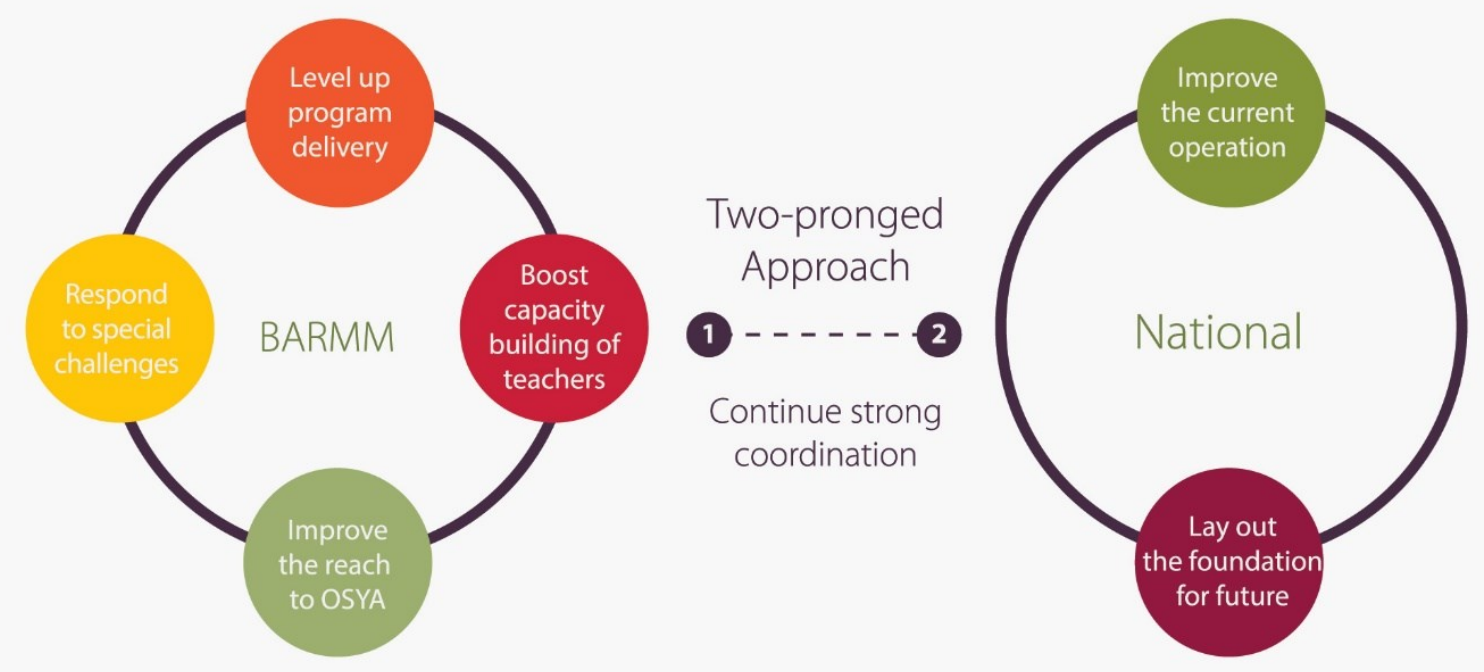

Source: Authors' analysis. 


\section{BARMM}

\section{Level up the Program Delivery}

A new BARMM roadmap to improve the ALS program delivery should be the important basis for leveling up the current operations. The ALM Roadmap lays out concrete actions with annual targets and should serve as the basis for implementation as well as monitoring and evaluation in the immediate and mid-term. Particularly, the actions suggested during the immediate term (particularly, the BARMM transition period) could be of the priority. Among significant supply-side bottlenecks, providing ALS facilitators with more ALS Learning Modules and ensuring operational allowances received by ALS facilitators on-time could be the immediate actions. Also, maximizing the use of existing public schools for ALS learning sessions could be promoted.

Synergizing efforts with other partners implementing the ALS programs in BARMM could be further promoted. As the short-term action, conducting joint planning, targeting, and prioritization of resources and M\&E; as well as sharing of resources, expertise, data and reporting with partners could be pursued. Fort this, continued leadership and support by the BARMM Education Ministry to provide strong accountability mechanisms at the region and field levels will remain critical.

In the medium term, the BARMM Ministry of Education could review ways to channel more resources directly and timely to the ALS implementers to address persistent deficiencies of learning resources on the ground. Investing a program that provides additional resources to ALS implementers will significantly improve learning facilities, make more learning materials (both national and localized) available to more learners, ease the burden on teachers to procure educational supplies, and deliver more opportunity for livelihood skill development for learners, which should keep them attracted to learning. With the incoming block grants for BARMM, as well as the inflow of support from development partners, the challenge for the government could now be absorptive capacity of the new government, and technical assistance to improve the financial management, budgeting, and supervision mechanism could be explored in the medium term.

\section{Boost Capacity Building of Teachers}

In the short- and medium-term, the BARMM Ministry of Education should intensify its efforts to deliver more quality training programs for the existing and incoming ALS facilitators. The teachers in BARMM generally lack opportunities for professional development, and a significant number of them will retire and be replaced by newly-hired teachers. As reported, pedagogical approach in ALS and the new contents from the K-12 Basic Education ALS curriculum could be of the priority focus for the training. The Mass Training of Teachers conducted in 2019 to prepare for the national roll-out of the ALS Version 2.0 provided a good baseline for capacity-building programs, which should be accompanied with more division-level follow-up trainings for all its ALS facilitators in BARMM.

In the medium term, localized peer-learning development programs could be carefully designed and introduced for ALS facilitators' continuous professional development in BARMM. A good model may be similar to the Learning Action Cell (LAC) sessions, which have been conducted in public schools across the country aiming to provide school-based in-service training and coaching for teachers. As ALS facilitators operate remotely and independently, LAC-based capacity building activities should be carefully designed and slowly introduced through a series of validations to further enhance the capability building structure, so that instructional supervision and support will be enhanced in ALS.

\section{Improve the reach to OSYA}

Also, in the short-term, the BARMM government could consider using a targeted approach to reach the accumulated OSYA with local partners effectively and efficiently. Utilizing the available microdata, 
the Community Mapping activity for ALS could focus on the areas where OSYAs are concentrated. This mapping could emphasize its advocacy impact among prospective ALS learners instead of generating the comprehensive data base, which has been challenging especially with limited manpower. Improved ALS outcomes, such as better completion rate and A\&E pass rate, will send good signals in advocating the program effectively. Moreover, local collaboration and partnership with the formal elementary and high schools, as well as local government units could be further sought for better advocacy.

One of the foremost tasks is to improve the image and advocacy of the formal school education and ALS holistically, with a special focus on girls. The critical challenges in ALS are closely linked to challenges in the formal education system, and there is urgent need for BARMM to strengthen the participation and survival of girls in the formal education, as well as female learners in ALS. Seizing the momentums of the BARMM transition and the ALS 2.0 roll-out, advocacy campaigns could send a new message that ALS is indeed a reliable complement towards quality education and personal competitiveness This effort could be carried out effectively in close coordination with the ALS Taskforce ${ }^{16}$ and need to be sustained for the medium- and long-term.

In the medium-term, possible financial schemes could be explored to increase the number of field implementers of the ALS programs. This may include resigning of the ALS Service Contracting Scheme or fitting a voucher programs linked to specific outcomes. These education service contracting schemes were implemented in ALS in other regions but have been suspended for review and revision for various reasons. ${ }^{17}$

\section{Respond to special and irregular practices}

The BARMM government should start strengthening its governance and field supervision urgently, to improve service delivery and eradicate irregular practices. An inventory of all approved ALS teaching items against the status of deployment accompanied with continuous random spot-checks of learning sessions by increasing participation of civil society groups and communities needs to be done. The use of technology could be utilized for this.

Regular program audits for verification should be carried out at least annually, and unannounced on-site monitoring should be sustained on a random basis in the medium- and long-term. Particularly in the areas where they see persistent and prevalent corruption issues, engagement with third-party agents such as civil society groups and local communities will be very critical.

\section{National Level}

\section{Improve the current operation}

Expanding public spending for ALS programs is critical to deliver quality ALS programs and support system-wide changes that will flow from the new ALS 2.0. The increased funding will be useful to alleviate significant deficiencies in teacher items, learning modules, capacity building, assessment and certification, advocacy and social mobilizations, governance and financial management, and better learning environments. More operating expenses for ALS teachers, in the way of teaching and transportation allowances will also provide practical support for the areas where the need is relatively more significant on the ground. The allocation of public spending should be evidence-based and accompany a monitoring mechanism.

Efforts by DepEd to provide a policy environment to harmonize the formal school education, ALS, and ADM within the overall context of Philippines' Basic Education will need to be sustained in the medium- and long-term. In particular, programs designed for school dropouts and high-risk students, 
specifically ADM, should be reviewed and intensified simultaneously with enhancing the ALS, in order to holistically address the challenges for the formal school systems and ALS.

\section{Layout the foundation for future}

Looking forward, it is critical for the national level to begin laying the foundation for the future of ALS and become more responsive to the requirements of the global economy. Among many priorities, the following three areas are currently missing or weak in ALS, which could potentially boost the ALS program in the medium and long terms:

- Recognition of Prior Learning (RPL): Recognizing their prior learning/skills and packaging this assortment of skills into something that will be valuable to employers and society could be further improved. The on-going RPL is often accomplished only for the sake of compliance, and most are rarely revisited to help calibrate the learning program as it progresses. Improvement in the implementation and monitoring of various assessments will help keep the learners motivated to continue their participation in the program.

- Career consultation or guidance support for ALS learners: Helping learners to make informed decisions about their future (especially if it includes support for on-the-job trainings and career placements) has been limited and could be further promoted. DepEd is very recently intensifying its career guidance and counseling program with the Department of Labor and Employment for secondary education. This post-ALS program needs to be anchored on coordination/alliance building with schools, other government line agencies, LGUs, and private sector partners. ${ }^{18}$

- Modular learning and micro-certification: In a longer-term, modular-based learning accompanied with micro-certification mechanisms could be explored and strengthened. ALS has long used learning modules that are self-contained in some ways, but the competencies these impart are not certified on an individual basis. Currently, validation of learning will only happen if the learner passes the A\&E test. This all-or-nothing situation serves as a psychological barrier to more participation in the program. Of course, modular learning is effective only if sufficient quantities of quality learning modules, along with the proper program design, are available to learners. With the expansion of the scope and number of competencies in the new ALS curriculum, proportionate investment in learning materials development, printing and distribution is needed to make "modular learning" operationally effective as an instructional delivery strategy. This area could be also learned from international good practices.

\section{Conclusion}

This study provided a comprehensive review of the ALS operation in BARMM based on new data collected in the field. ALS has been the only government program widely implemented in BARMM to help low-skilled and low-educated youths and adults (including ex-combatants) who missed formal school education for various reasons. However, the majority of the findings suggest that the program has not yet reached its full potential in BARMM. The evidence from this study should be useful for the new BARMM government to design necessary interventions and set concrete targets that will guide a new reform to improve the quality of ALS programs on the short, medium and long run.

The roll-out of a new ALS K-12 curriculum, which is aligned to the K-12 Basic Education Curriculum, or known as "ALS Version 2.0", has been an opportunity to revisit and upgrade the system components of ALS throughout the country. The full transition to the new BARMM government, as well as the national roll-out of the "ALS Version 2.0", should offer a unique opportunity for BARMM to work on these challenges in the short run and set key directions for the future, in order to unlock the full potential of people in BARMM. 


\section{BARMM Level up the overall ALS program delivery}

- Implement the BARMM Alternative Learning Modalities (ALM) ${ }^{21}$ Strategic Roadmap

- Explore ways to ensure more resources disbursed

- Liaise with DepEd to improve the availability of ALS Learning Modules and the condition of existing ALS learning centers

- Improve the coordination with non-DepEd ALS implementers, especially in M\&E

\section{Boost capacity building programs for ALS teachers}

- Improve delivery and quality of the existing training programs covering more ALS implementers in collaboration with DepEd

- Promote localized peer-learning programs (e.g. LAC in ALS) and improve continuous career development programs for ALS implementers

\section{Improve approaches to reach out to OSYA}

- Introduce a targeted approach in conducting community mapping and advocacy exercises, in collaboration with local partners (e.g. schools, CSO, LGU, and other GAs)
- Explore interventions to improve the image of the entire education sector, especially those to support girls and female learners attending formal schools and ALS

- Explore viable schemes to expand the delivery of ALS programs (e.g. voucher or service contracting schemes)

\section{National Improve the delivery of quality ALS programs}

- Expand public spending for the ALS program, focusing in the first instance on increasing the supply of learning modules

- Execute and monitor the implementation of the ALS 2.0 Five-Year Strategic Roadmap
- Provide a policy environment which will harmonize the formal school education with ALS and ADM by defining target beneficiaries and eligibility conditions

\section{Lay out foundations to strengthen ALS for future}

- Develop a program for ALS implementers on providing post-ALS career guidance for ALS learners
- Develop micro-certification mechanisms for adult learners and modular-based learning systems while intensifying the use of digital technology

- Strengthen quality of implementing the Recognition of Prior Learning in line with the K-12 BEC-aligned ALS curriculum 


\section{References}

Acosta, P. A., Igarashi, T., Olfindo, R., \& Rutkowski, J. J. 2017. Developing Socioemotional Skills for the Philippines’ Labor Market (English). Washington, DC: World Bank Group.

Aker, Jenny C., Christopher Ksoll, and Travis J. Lybbert. 2012. "Can Mobile Phones Improve Learning? Evidence from a Field Experiment in Niger." American Economic Journal: Applied Economics 4 (4): 94-120.

Aker, Jenny C. and Melita Sawyer. 2017. “Adult Learning in Sub-Saharan Africa: What Do and Don't We Know?" Forthcoming.

Al-Samarrai, S. 2016. Assessing Basic Education Service Delivery in the Philippines: Public Education Expenditure Tracking and Quantitative Service Delivery Study (English). Washington, DC: World Bank Group. http://documents.worldbank.org/curated/en/507531468325807323/Assessing-basic-educationservice-delivery-in-the-Philippines-public-education-expenditure-tracking-and-quantitativeservice-delivery-study.

Angel, Gloria Miranda; Fernandez, Maria Carmen Ica; Kandiwal, Wali Mohammad; Moro, Leben; and Hollway, Kerrie. (2019). Dignity in displacement. Case studies from Afghanistan, Colombia, the Philippines and South Sudan. Humanitarian Policy Group (HPG) Working Paper. March 2019. ODI.

Banerji, Rukmini, James Berry, and Marc Shotland. (2017). "The Impact of Maternal Literacy and Participation Programs: Evidence from a Randomized Evaluation in India." American Economic Journal: Applied Economics 9 (4): 303-337.

Blunch, Niels-Hugo, and Claus C. Pörtner. (2011). Literacy, Skills, and Welfare: Effects of Participation in Adult Literacy Programs. Economic Development and Cultural Change, 60(1), 17-66. doi:10.1086/661219

David, Clarissa and Albert, Jose Romon. (2015). Recent Trends in Out-of-School Children in the Philippines. Discussion Paper Series. No. 2015-51. Philippines Institute for Development Studies.

Department of Education Various years. Enhanced Basic Education Information System. Republic of the Philippines.

Department of Education Various years. Learner Information System. Republic of the Philippines.

Department of Budget and Management. Various years. Republic of the Philippines.

Department of Foreign Affairs and Trade. (2017). Philippines Basic Education Assistance for Mindanao (BEAM) in the Autonomous Region in Muslim Mindanao: end of program review. Cotabato City, Philippines. https://dfat.gov.au/about-us/publications/Pages/philippines-beam-armm-end-ofprogram-review.aspx

Department of Foreign Affairs and Trade. (2018). Preliminary Investigation on Unserved Barangays. Cotabato City, Philippines. 
Halasz, G., Sweet, R., Gallagher, M., \& Tuomi, O. 2009. Recognition of Non-formal and Informal Learning: Country Note for South Korea. Paris: OECD Publishing.

Hanemann U. (Ed.). Last update: 21 July 2017. Education Model for Life and Work, Mexico. UNESCO Institute for Lifelong Learning. (Accessed on: 9 October 2019, 22:42 CEST)

Heckman, James J., John Eric Humphries, and Nicholas S. Mader. 2011. “The GED.” NBER (National Bureau of Economic Research) Working Paper 16064.

Heckman, James J., John Eric Humphries, Paul A. LaFontaine, and Pedro L. Rodriguez. 2012. “Taking the Easy Way Out: How the GED Testing Program Induces Students to Drop Out." Journal of Labor Economics 30 (3): 495-520.

Herlyna, H., Mukhtar, M., \& Sari, E. (2019). The Evaluation of Paket C Equivalency Program Implementation in Tangerang District, Indonesia.

Igarashi, T. 2018. A Second Chance to Develop the Human Capital of Out-of-School Youth and Adults: The Philippines Alternative Learning System (English). Washington, DC: World Bank Group.

Knowles, Malcolm S. 1980. "The Modern Practice of Adult Education, From Pedagogy to Andragogy." Cambridge Adult Education.

Lee, H. Y., Koh, Y. S., Park, S. O. and Park, S. M. 2010. Manual for the Recognition of Prior Learning. Seoul: National Institute for Lifelong Education.

National Institute for Lifelong Education. 2013. Manual for the Academic Credit Bank System. Seoul: National Institute for Lifelong Education.

Nedelkoska, L., \& G. Quintini. 2018. Automation, Skill Use and Training. OECD Social, Employment and Migration Working Papers No. 202. Paris: OECD Publishing.t:

http://dx.doi.org/10.1787/2e2f4eea-en.

OECD. 2017. OECD Skills Outlook 2017: Skills and Global Value Chains. Paris: OECD Publishing. https://dx.doi.org/10.1787/9789264273351-en.

OECD. 2019. Getting Skills Right: Future-Ready Adult Learning Systems. Paris: OECD Publishing. https://doi.org/10.1787/9789264311756-en.

Philippine Statistics Authority. 2013. Functional Literacy, Education and Mass Media Survey.

Rajasekaran, Subhashini; Reyes, Joel E.. 2019. Back to School : Pathways for Reengagement of Out-ofSchool Youth in Education (English). International Development in Focus. Washington, D.C. : World Bank Group.

Sawada, Yasuyuki, Minhaj Mahmud, Mai Seki, An Le, and Hikaru Kawarazaki. 2017. "Individualized Self-Learning Program to Improve Primary Education: Evidence from a Randomized Field Experiment in Bangladesh.” JICA-RI (Japan International Cooperation Agency Research Institute) Working Paper 156.

Singh, Madhu; Idris, Hegazi; and Chehab, Maysoun.2018. "Pathways to empowerment: Recognizing the competences of Syrian refugees in Egypt, Iraq, Jordan, Lebanon and Turkey." UNESCO Institute for Lifelong Learning. 
SEAMEO INNOTECH. 2015. "Evaluation of the Open High School Program in the Philippines." Quezon City, the Philippines.

Thomas, M. S. C., Ansari, D., \& Knowland, V. 2019. Educational Neuroscience: Progress and Prospects. Journal of Child Psychology and Psychiatry 60(4), 477-492.

UNESCO (United Nations Educational, Scientific, and Cultural Organization). 2015. Education for All 2000-2015: Achievements and Challenges. EFA Global Monitoring Report.

Usher, A. 2014. The Korean Academic Credit Bank: A Model for Credit Transfer in North America? Toronto: Higher Education Strategy Associates.

Velarde, R. A. 2018. The Philippines' Targeting System for the Poor: Successes, Lessons, and Ways Forward (English). World Bank Social Protection Policy Note No. 16. Washington, DC: World Bank Group.

Windisch, H. (2015), "Adults with low literacy and numeracy skills: A literature review on policy intervention”, OECD Education Working Papers, No. 123, OECD Publishing, Paris.

World Bank. The World Bank Projects and Operations: Mindanao Trust Fund-Reconstruction and Development Project Phase II. http://projects.worldbank.org/P164019?lang=en

World Bank. 2015. Making Education Spending Count for the Children of Autonomous Muslim Region of Mindanao: Public Expenditure and Institutional Review for BARMM Basic Education (English). Washington, DC: World Bank Group.

Yamauchi, F., Igarashi, T., Tenazas, N. M., \& Tiongco, M. M. 2016. Alternative and Inclusive Learning in the Philippines (English). Washington, DC: World Bank Group.

Yang, J. 2015. Recognition, Validation and Accreditation of Non-formal and Informal Learning in UNESCO Member States. Hamburg: UNESCO Institute for Lifelong Learning. 


\section{Endnotes}

${ }^{1}$ The data collection for this study was undertaken between November 2018 and February 2019. The survey was designed based on the jurisdiction of ARMM (former BARMM). The official data such as DepEd, DBM, and PSA used in the report is of SY2017/2018 or before. Please note, that the study uses Bangsamoro Autonomous Region in Muslim Mindanao, or BARMM in short, consistently to call the region to avoid confusion for those who are fully familiar with the political process. The region has been in the transition process with the Bangsamoro Organic Law (BOL), which was signed on July 31, 2018 by President Duterte, and the plebiscites in January and February 2019.

${ }^{2}$ ALS programs have been typically delivered from January to October in a year, followed by the A\&E exam. With the full introduction of the K-12 basic education program and its curriculum, the ALS curriculum has been aligned to the competencies required in the formal system. Accordingly, particularly the A\&E secondary program duration are extended to a longer duration. It is important to keep in mind that ALS remains as "open" programs which accepts enrollees at any time throughout a year.

${ }^{3}$ The 2013 Enhanced Basic Education set the foundational framework of the Philippines basic education system. The new system was rolled out gradually, which was completed with introduction of Senior High Schools in 2016.

${ }^{4}$ For more details, see OECD (2019).

${ }^{5}$ The survey team (WB, DepEd, and CBCS) undertook a series of field tests to understand the local contexts and incorporated them into the survey instruments in Maguindanao and Cotabato City.

${ }^{6}$ Including national household surveys, and other studies conducted by WB and others

${ }^{7}$ Because of the increased security concerns in January 2019, the survey was conducted mainly in Jolo (and a few additional locations) and only for Sulu Province.

${ }^{8}$ In 2010 and 2011, ARMM's NER exceeded 100 and the national average, reflecting inconsistencies in the school information data and/or population data, which have disappeared to date.

${ }^{9}$ Included only elementary and junior high school education as SHS is not offered for ALS. JHS completion age is 16 , but 2 years is factored in the official JHS completion age given high share of average students who are still coming up with studies in school.

${ }^{10}$ The DepEd data showed extreme levels of fluctuation in the enrollment. For example, the enrollment increased by 280 percent (3,500 in 2007 to 13,600 in 2008) or by 390 percent (2,800 in 2013 to 14,000 in 2014) or decreased by 50 years (19,000 in 2011 to 10,000 in 2012), which may not be so realistic. Most probably, there might have been issues of submission and consolidation of the data between central and sub-national levels.

${ }^{11}$ Based on the background questions in the Programme for the International Assessment of Adult Competencies (PIAAC), a programme of assessment and analysis of adult skills was administered by OECD.

${ }^{12}$ The low participation in adult learning programs is explained by reluctance and difficulty in recognizing their learning needs and seeking out learning opportunities (OECD, 2018; Windisch, 2015).

${ }^{13}$ Note that this share of A\&E passers computed using the survey data is different or lower that the passing rate reported by the Bureau of Educational Assessment, DepEd. The share of passers is based on the number of ALS enrollers in the same year while the DepEd data is based on the number of the A\&E test takers and does not differentiate whether they had the ALS intervention in the same year or not (including the walk-in and those attempting the test multiple times). This does not mean that those who had interventions had more non-passers but simply rather that the denominators used to derive these shares are different; thus, they can't be compared.

${ }^{14}$ Person who can engage in all those activities in which literacy is required for effective function of his or her group and community and also for enabling him or her to continue to use reading, writing and calculation for his or her own and the community's development. (UNESCO)

${ }^{15}$ To be rolled out in June 2019 across the Philippines including BARMM.

${ }^{16}$ Deped order on ALS TF. DepEd Order No. 3, Series 2019.

${ }^{17}$ This contracting scheme could address the issues surfaced with the old scheme, while considering the unique context in BARMM. Improvements in this scheme may include: (1) more sensible contract amounts, (2) output-based deliverables with possible performance bonuses, (3) rational geographic assignments, (4) operationalization of team teaching, including certified TVET trainers, (5) stronger monitoring and performance evaluation, and (6) regular programmatic audits.

${ }^{18}$ It cannot be limited within DepEd alone. One model has been piloted in the USAID MYDev as Youth Development Alliance. 